\title{
Uranium Metallocene Azides, Isocyanates, and Their Borane-Capped Lewis Adducts
}

Michael A. Boreen, ${ }^{\S, \dagger}$ Karl N. McCabe, ${ }^{\Delta}$ Trevor D. Lohrey, ${ }^{\S, \dagger}$ Fabian A. Watt, ${ }^{\#}$ Laurent Maron, ${ }^{\Delta}$ Stephan Hohloch, ${ }^{\cap, *}$ and John Arnold ${ }^{\S, \dagger, *}$

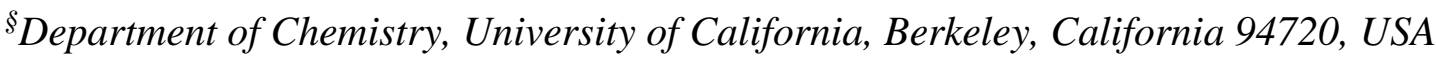

${ }^{\dagger}$ Chemical Sciences Division, Lawrence Berkeley National Laboratory, Berkeley, California 94720, USA

${ }^{4}$ LPCNO, Université de Toulouse, INSA Toulouse, 135 Avenue de Rangueil, 31077 Toulouse, France

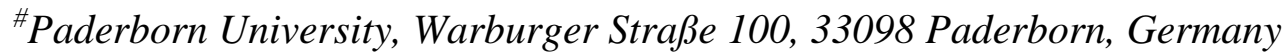

${ }^{n}$ University of Innsbruck, Faculty of Chemistry and Pharmacy, Institute of General, Inorganic and Theoretical Chemistry, Innrain 80-82, 6020 Innsbruck, Austria.

*Email: arnold@ berkeley.edu, stephan.hohloch@uibk.ac.at

\section{Supporting Information}

Experimental Procedures

Figures S1-S3

Schemes S1-S4

S8-S9

NMR Spectroscopy

S10-S21

X-ray Crystallography

Electrochemistry

S35-S36

UV-Visible Spectroscopy

Computational Section

S38-S81

References

S82-S84 


\section{Experimental Procedures}

General Considerations: Unless otherwise noted, all reactions were performed using standard Schlenk line techniques under an atmosphere of nitrogen or argon or in an MBraun inert atmosphere glove box under an atmosphere of nitrogen. Glassware and Celite ${ }^{\circledR}$ were stored in an oven at $c a .150{ }^{\circ} \mathrm{C}$ for at least $3 \mathrm{~h}$ prior to use. Molecular sieves $(4 \AA)$ were activated by heating to $200{ }^{\circ} \mathrm{C}$ overnight under vacuum prior to storage in a glove box. NMR spectra were recorded at room temperature unless noted otherwise on Bruker AV-300, AVB-400, AVQ-400, DRX-500, AV-500, or AV-600 spectrometers. ${ }^{1} \mathrm{H}$ NMR chemical shifts $(\delta)$ are given relative to residual solvent peaks and are recorded in units of parts per million (ppm). ${ }^{19} \mathrm{~F}$ NMR chemical shifts $(\delta)$ are reported in ppm and referenced to an external standard $\left(\mathrm{CFCl}_{3}\right.$ in $\left.\mathrm{CDCl}_{3}\right)$. Where peaks could be fitted, full width at half maximum (FWHM) values are reported in Hertz as determined by leastsquares fitting of data to Lorentzian line shapes in MestReNova (version 10.0.2-15465). Default $(0.3 \mathrm{~Hz})$ line broadening functions were applied to ${ }^{1} \mathrm{H}$ NMR spectra unless stated otherwise. FTIR samples were prepared as Nujol mulls pressed between $\mathrm{KBr}$ plates, with data collected with a Nicolet iS10 FT-IR spectrometer. Melting points were determined using sealed capillaries prepared under nitrogen on an OptiMelt automated melting point system. Elemental analyses were determined at the Microanalytical Facility at the College of Chemistry, University of California, Berkeley. UV-Vis measurements were performed on a Varian Cary® 50 UV-Vis Spectrophotometer. A two mm path length quartz cell was used, and a blank was subtracted from each run. Photolysis experiments were conducted in a Rayonet reactor, model RPR-100, centered at $253 \mathrm{~nm}$.

Materials: Diethyl ether, $n$-hexane, $n$-pentane, THF, and toluene were purified by passage through columns of activated alumina and degassed by sparging with nitrogen. Hexamethyldisiloxane (HMDSO) and $\mathrm{C}_{6} \mathrm{D}_{6}$ were vacuum-transferred from flasks containing sodium/benzophenone and stored over molecular sieves. $\left(\mathrm{Cp}^{\mathrm{iPr} 4}\right)_{2} \mathrm{UI},{ }^{1}\left(\mathrm{Cp}^{\mathrm{iPr} 4}\right)_{2} \mathrm{UI}_{2},{ }^{1} \mathrm{~B}\left(\mathrm{C}_{6} \mathrm{~F}_{5}\right)_{3},{ }^{2}\left[{ }^{n} \mathrm{Bu} 4 \mathrm{~N}\right]\left[\mathrm{B}\left(\mathrm{C}_{6} \mathrm{~F}_{5}\right)_{4}\right],{ }^{3,4}$ and $\left[\left(\mathrm{Cp}^{\mathrm{iPr} 4}\right)_{2} \mathrm{U}\left(\mu-\eta^{1}: \eta^{1}-\mathrm{N}_{3}\right)\right]_{4} \quad\left(\mathbf{3}-\mathrm{N}_{3}\right)^{5}$ were synthesized according to literature procedures. $\left[{ }^{n} \mathrm{Bu}_{4} \mathrm{~N}\right][\mathrm{OCN}]$ was recrystallized from THF prior to use. Cesium fluoride (CsF) was dried in vacuo at $150{ }^{\circ} \mathrm{C}$ for $2 \mathrm{~h}$. All other chemicals were purchased from commercial sources and used as received.

Caution! Depleted uranium (primarily ${ }^{238} \mathrm{U}$ ) is an alpha-emitter with a half-life of $4.47 \times 10^{9}$ years. Manipulations were carried out in fume hoods or in inert atmosphere glove boxes within radiological laboratories using appropriate personal protective and $\alpha$ - and $\beta$-counting equipment.

\section{Synthesis of $\left(\mathrm{Cp}^{\mathrm{iPr} 4}\right)_{2} \mathrm{U}\left(\mathrm{N}_{3}\right)_{2}\left(\mathbf{1 - N _ { 3 }}\right)$}

THF ( $2 \mathrm{~mL})$ was added to a mixture of $\left(\mathrm{Cp}^{\mathrm{iPr} 4}\right)_{2} \mathrm{UI}_{2}\left(43 \mathrm{mg}, 0.045 \mathrm{mmol}, 1.0\right.$ equiv) and $\mathrm{NaN}_{3}(7.2$ $\mathrm{mg}, 0.11 \mathrm{mmol}, 2.5$ equiv), and the resulting red suspension was stirred for $18 \mathrm{~h}$ at room temperature. Volatiles were removed in vacuo, and the product was extracted into $4 \mathrm{~mL}$ of hexane, 
filtered through Celite, concentrated, and cooled to $-40{ }^{\circ} \mathrm{C}$. The product was isolated as red crystals and dried in vacuo $(29 \mathrm{mg}, 0.037 \mathrm{mmol}, 81 \%$ yield). X-ray quality crystals of 1-N3 were grown from the slow evaporation of a hexane solution at room temperature. Mp ca. $199{ }^{\circ} \mathrm{C}$ (decomp.); ${ }^{1} \mathrm{H}$ NMR (500 MHz, $\mathrm{C}_{6} \mathrm{D}_{6}$ ): $\delta 56.1$ to $37.7,37.7$ to $25.3,25.3$ to $12.9,12.9$ to -17 (this signal is particularly difficult to identify as it appears to overlap a considerable amount of the diamagnetic region), -25.7 to $-35.5,-43.5$ to -62.0 ; assignments of chemical shift ranges are tentative due to the intrinsic extremely broad nature of the signals and the related inability to correct the baseline of the spectrum; IR: $v\left(\mathrm{~N}_{3}\right) 2096$ (vs), 2082 (vs) cm ${ }^{-1}$. Anal. Calcd. (\%) for $\mathrm{C}_{34} \mathrm{H}_{58} \mathrm{~N}_{6} \mathrm{U}$ (1-N): C, 51.76; H, 7.41; N, 10.65. Found: C, 51.42; H, 7.47; N, 10.57.

\section{Synthesis of $\left(\mathrm{Cp}^{\mathrm{iPr} 4}\right)_{2} \mathrm{U}(\mathrm{NCO})_{2}(\mathbf{1 - N C O})$}

Method A. A solution of [ $\left.{ }^{n} \mathrm{Bu} 4 \mathrm{~N}\right][\mathrm{OCN}](66 \mathrm{mg}, 0.23 \mathrm{mmol}, 2.2$ equiv) in THF (2 mL) was added dropwise to a solution of $\left(\mathrm{Cp}^{\mathrm{iPr} 4}\right)_{2} \mathrm{UI}_{2}(101 \mathrm{mg}, 0.106 \mathrm{mmol}, 1.0$ equiv) in THF $(1 \mathrm{~mL})$, and the resulting solution was stirred for $24 \mathrm{~h}$ at room temperature. During this time, the reaction mixture changed from dark red to red-orange, and colorless solid precipitated. Volatiles were removed in vacuo, and the product was extracted into $10 \mathrm{~mL}$ of hexane, filtered through Celite, concentrated, and cooled to $-40{ }^{\circ} \mathrm{C}$. The product was isolated as red crystals and dried in vacuo. Concentration and cooling of the supernatant yielded a second crop of product in a similar fashion $(60 \mathrm{mg}$ combined, $0.076 \mathrm{mmol}, 72 \%$ yield). X-ray quality crystals of 1-NCO were grown from the slow evaporation of a hexane solution at room temperature. Mp ca. $213{ }^{\circ} \mathrm{C}$ (decomp.); ${ }^{1} \mathrm{H}$ NMR (500 $\mathrm{MHz}, \mathrm{C}_{6} \mathrm{D}_{6}$ ): $\delta 49.1$ to $38.6,38.6$ to $27.7,27.7$ to $12.4,12.4$ to -13 (this signal is particularly difficult to identify as it appears to overlap a considerable amount of the diamagnetic region), -20.5 to $-30.4,-30.4$ to $-37.6-43.9$ to -68.2 ; assignments of chemical shift ranges are tentative due to the intrinsic extremely broad nature of the signals and the related inability to correct the baseline of the spectrum; IR: $v$ (NCO) 2199 (vs), 2181 (vs) $\mathrm{cm}^{-1}$. Anal. Calcd. (\%) for $\mathrm{C}_{36} \mathrm{H}_{58} \mathrm{~N}_{2}$ $\mathrm{O}_{2} \mathrm{U}$ (1-NCO): C, 54.81; H, 7.41; N, 3.55. Found: C, 54.63; H, 7.63; N, 3.48.

Method B. Toluene (4 mL) was added to 3-OCN (25 mg, $0.0084 \mathrm{mmol}$ ) in a $25 \mathrm{~mL}$ glass flask with a Teflon seal. The flask was sealed, and the suspension was heated to $150{ }^{\circ} \mathrm{C}$ with stirring for $18 \mathrm{~h}$. During this time, the reaction mixture changed from a blue-green suspension to a clear, orange solution. The solution was allowed to cool to room temperature, then volatiles were removed in vacuo. The product was extracted into $1 \mathrm{~mL}$ of pentane, filtered through Celite, concentrated, and cooled to $-40{ }^{\circ} \mathrm{C}$. The product was isolated as red crystals and dried in vacuo $(6.3 \mathrm{mg}, 0.0080 \mathrm{mmol}, 47 \%$ yield based on expected 0.5 equiv of product relative to each equiv of uranium in 3-OCN). The ${ }^{1} \mathrm{H}$ NMR spectrum of the isolated material matched that of analytically pure 1-NCO synthesized by Method A.

\section{Synthesis of $\left(\mathrm{Cp}^{\mathrm{iPr} 4}\right)_{2} \mathrm{U}(\mathrm{OTf})_{2}$ (1-OTf)}

A solution of AgOTf (97 mg, $0.38 \mathrm{mmol}, 2.2$ equiv) in ether (4 mL) was added dropwise to a solution of $\left(\mathrm{Cp}^{\mathrm{iPr} 4}\right)_{2} \mathrm{UI}$ (143 mg, $0.171 \mathrm{mmol}, 1.0$ equiv) in ether $(2 \mathrm{~mL})$, and the resulting dark red 
solution with gray precipitate was stirred in the dark for $3 \mathrm{~h}$ at room temperature. Volatiles were removed in vacuo, and the product was extracted into $10 \mathrm{~mL}$ of hexane, filtered through Celite, concentrated, and cooled to $-40{ }^{\circ} \mathrm{C}$. The product was isolated as dark red crystals and dried in vacuo (147 mg, $0.147 \mathrm{mmol}, 86 \%$ yield). X-ray quality crystals of 1-OTf were grown from a solution of toluene/HMDSO (4:1 ratio) at $-40{ }^{\circ} \mathrm{C}$. Mp ca. $143{ }^{\circ} \mathrm{C}$ (decomp.); ${ }^{1} \mathrm{H} \mathrm{NMR} \mathrm{(500} \mathrm{MHz,}$ $\mathrm{C}_{6} \mathrm{D}_{6}$ ): $\delta 82.64$ (broad, 2H, $455 \mathrm{~Hz}$ ), 52.74 (broad, 2H, $449 \mathrm{~Hz}$ ), 48.00 (broad, 6H, $390 \mathrm{~Hz}$ ), 36.45 (broad, 6H, $350 \mathrm{~Hz}$ ), 25.63 (broad, 6H, 390 Hz), 18.72 (broad, 6H, $328 \mathrm{~Hz}$ ), 16.52 (broad, 2H, 516 Hz), 14.48 (broad, 6H, 333 Hz), -20.80 (broad, 2H, 347 Hz), -24.46 (broad, 6H, 388 Hz), -40.68 (broad, 6H, $342 \mathrm{~Hz}$ ), -64.72 (broad, 2H, $241 \mathrm{~Hz}$ ), -80.69 (broad, 6H, $432 \mathrm{~Hz}) ;{ }^{19} \mathrm{~F}$ NMR (376 $\left.\mathrm{MHz}, \mathrm{C}_{6} \mathrm{D}_{6}\right) \delta-103.76\left(60.2 \mathrm{~Hz}\right.$ ). Anal. Calcd. (\%) for $\mathrm{C}_{36} \mathrm{H}_{58} \mathrm{~F}_{6} \mathrm{O}_{6} \mathrm{~S}_{2} \mathrm{U}$ (1-OTf): C, 43.11; H, 5.83. Found: C, 43.22; H, 5.88.

\section{Synthesis of $\left(\mathrm{Cp}^{\mathrm{iPr} 4}\right)_{2} \mathrm{U}\left(\mathrm{N}_{3}\right)(\mathrm{OTf})(\mathbf{1 - N} 3-\mathrm{OTf})$}

Toluene $(6 \mathrm{~mL})$ was added to a mixture of 1-OTf (73 mg, $0.072 \mathrm{mmol}, 1.0$ equiv) and 1-N3 (57 $\mathrm{mg}, 0.073 \mathrm{mmol}, 1.0$ equiv) in a $25 \mathrm{~mL}$ glass flask with a Teflon seal. The flask was sealed, and the solution was heated to $65{ }^{\circ} \mathrm{C}$ with stirring for $24 \mathrm{~h}$. Volatiles were removed in vacuo, and the product was extracted into $3 \mathrm{~mL}$ of toluene, filtered through Celite, concentrated, and cooled to $40{ }^{\circ} \mathrm{C}$. The product was isolated as dark red crystals after washing with $1 \mathrm{~mL}$ of pentane and drying in vacuo (86 mg, $0.096 \mathrm{mmol}, 67 \%$ yield). X-ray quality crystals of 1-N3-OTf were grown from pentane at room temperature. Mp ca. $166{ }^{\circ} \mathrm{C}$ (decomp.); ${ }^{1} \mathrm{H} \mathrm{NMR}\left(400 \mathrm{MHz}, \mathrm{C}_{6} \mathrm{D}_{6}\right): \delta 69.3$ to $50.0,50.0$ to $36.5,36.5$ to $24.4,24.4$ to 8.5 (this signal appears to extend further upfield into or beyond the diamagnetic region), -15.6 to $-31.6,-31.6$ to -65.4 ; assignments of chemical shift ranges are tentative due to the intrinsic extremely broad nature of the signals and the related inability to correct the baseline of the spectrum; ${ }^{19} \mathrm{~F}$ NMR $\left(376 \mathrm{MHz}, \mathrm{C}_{6} \mathrm{D}_{6}\right) \delta-105.59(254 \mathrm{~Hz})$; IR: $v\left(\mathrm{~N}_{3}\right) 2098$ (vs) $\mathrm{cm}^{-1}$. Anal. Calcd. (\%) for $\mathrm{C}_{35} \mathrm{H}_{58} \mathrm{~F}_{3} \mathrm{~N}_{3} \mathrm{O}_{3} \mathrm{SU}$ (1-N3-OTf): C, 46.92; H, 6.53; N, 4.69. Found: C, 47.14; H, 6.63; N, 4.47.

\section{Synthesis of $\left(\mathrm{Cp}^{\mathrm{iPr}}\right)_{2} \mathrm{U}\left(\mathrm{N}_{3}\right)\left[\left(\boldsymbol{\mu}-\boldsymbol{\eta}^{\mathbf{1}}: \boldsymbol{\eta}^{\mathbf{1}}-\mathrm{N}_{\mathbf{3}}\right) \mathrm{B}\left(\mathrm{C}_{6} \mathrm{~F}_{\mathbf{5}}\right)_{3}\right]\left(\mathbf{2}-\mathrm{N}_{\mathbf{3}}\right)$}

A solution of $\mathrm{B}\left(\mathrm{C}_{6} \mathrm{~F}_{5}\right)_{3}(16 \mathrm{mg}, 0.031 \mathrm{mmol}, 1.0$ equiv) in pentane $(2 \mathrm{~mL})$ was added dropwise to a suspension of $\mathbf{1 - N _ { 3 }}(24 \mathrm{mg}, 0.031 \mathrm{mmol}, 1.0$ equiv) in pentane $(1 \mathrm{~mL})$. The resulting red suspension was stirred for $30 \mathrm{~min}$ at room temperature. Volatiles were removed in vacuo, and the products was extracted into $1 \mathrm{~mL}$ of ether, filtered through Celite, concentrated, and cooled to -40 ${ }^{\circ} \mathrm{C}$. The product was isolated as dark red crystals and dried in vacuo (34 mg, $0.026 \mathrm{mmol}, 87 \%$ yield). X-ray quality crystals of $\mathbf{2}-\mathbf{N}_{3}$ were grown from the slow evaporation of a hexane solution at room temperature. Mp ca. $165{ }^{\circ} \mathrm{C}$ (decomp.); ${ }^{1} \mathrm{H}$ NMR (400 MHz, $\mathrm{C}_{6} \mathrm{D}_{6}$ ): $\delta 53.6$ to 38.6, 38.6 to $27.5,27.5$ to $13.5,-20.0$ to $-39.7,-42.9$ to -65.3 (given the extreme broadness of these signals, the assigned ranges are approximate); ${ }^{19} \mathrm{~F}$ NMR (376 MHz, $\left.\mathrm{C}_{6} \mathrm{D}_{6}\right): \delta-149.90(\mathrm{~d}, J=13 \mathrm{~Hz})$, -157.49 (t, $J=21 \mathrm{~Hz}),-167.08(\mathrm{t}, J=18 \mathrm{~Hz})$; IR: $v\left(\mathrm{~N}_{3}\right) 2188(\mathrm{vs}), 2094$ (vs) cm ${ }^{-1}$. Anal. Calcd. (\%) for $\mathrm{C}_{52} \mathrm{H}_{58} \mathrm{BF}_{15} \mathrm{~N}_{6} \mathrm{U}\left(\mathbf{2}-\mathrm{N}_{3}\right)$ : C, 48.01; H, 4.49; N, 6.46. Found: C, 47.84; H, 4.40; N, 6.30. 


\section{Synthesis of $\left(\mathrm{Cp}^{\mathrm{iPr} 4}\right)_{2} \mathrm{U}(\mathrm{NCO})\left[\left(\mu-\eta^{\mathbf{1}}: \boldsymbol{\eta}^{\mathbf{1}}-\mathrm{OCN}\right) \mathrm{B}\left(\mathrm{C}_{6} \mathrm{~F}_{5}\right)_{3}\right](\mathbf{2}-\mathrm{NCO})$}

A solution of $\mathrm{B}\left(\mathrm{C}_{6} \mathrm{~F}_{5}\right)_{3}(16 \mathrm{mg}, 0.030 \mathrm{mmol}, 1.0$ equiv) in toluene $(2 \mathrm{~mL})$ was added dropwise to a solution of 1-NCO (24 mg, $0.030 \mathrm{mmol}, 1.0$ equiv) in toluene $(2 \mathrm{~mL})$. The resulting dark red solution was stirred for $24 \mathrm{~h}$ at room temperature. Volatiles were removed in vacuo, and the crude material was triturated with $1 \mathrm{~mL}$ of pentane to remove residual toluene. The products were then extracted into $3 \mathrm{~mL}$ of ether, filtered through Celite, concentrated, and cooled to $-40{ }^{\circ} \mathrm{C}$. The product was isolated as dark red crystals after washing with $0.5 \mathrm{~mL}$ of pentane and drying in vacuo (31 mg, $0.024 \mathrm{mmol}, 78 \%$ yield). X-ray quality crystals of 2-NCO were grown from the slow evaporation of a hexane solution at room temperature. Mp ca. $184{ }^{\circ} \mathrm{C}$ (decomp.); ${ }^{1} \mathrm{H}$ NMR (400 $\mathrm{MHz}, \mathrm{C}_{6} \mathrm{D}_{6}$ ): $\delta 55.1$ to $41.0,41.0$ to $30.7,30.7$ to $12.0,-21.3$ to $-44.4,-48.2$ to -72.0 (given the extreme broadness of these signals, the assigned ranges are approximate); ${ }^{19} \mathrm{~F}$ NMR (376 MHz, $\left.\mathrm{C}_{6} \mathrm{D}_{6}\right): \delta-146.57(\mathrm{dd}, J=24,8 \mathrm{~Hz}),-159.25(\mathrm{t}, J=21 \mathrm{~Hz}),-167.38(\mathrm{t}, J=22 \mathrm{~Hz}) ; \mathrm{IR}: v(\mathrm{NCO})$ 2354 (vs), 2193 (vs) cm ${ }^{-1}$. Anal. Calcd. (\%) for $\mathrm{C}_{54} \mathrm{H}_{58} \mathrm{BF}_{15} \mathrm{~N}_{2} \mathrm{O}_{2} \mathrm{U}$ (2-NCO): C, 49.86; H, 4.49; N, 2.15. Found: C, 50.09; H, 4.25; N, 2.01 .

\section{Synthesis of $\left[\left(\mathrm{Cp}^{\mathrm{iPr} 4}\right)_{2} \mathrm{U}\left(\mu-\eta^{1}: \eta^{\mathbf{1}}-\mathrm{NCO}\right)\right]_{4}(3-\mathrm{OCN})$}

THF (4 mL) was added to a mixture of $\left(\mathrm{Cp}^{\mathrm{iPr} 4}\right)_{2} \mathrm{UI}(123 \mathrm{mg}, 0.147 \mathrm{mmol}, 1.0$ equiv) and $\mathrm{NaOCN}$ (10 $\mathrm{mg}, 0.16 \mathrm{mmol}, 1.1$ equiv), and the resulting blue-green suspension was stirred for $18 \mathrm{~h}$ at room temperature. Volatiles were removed in vacuo, and the product was extracted into $4 \mathrm{~mL}$ of hexane (added quickly to ensure the product did not begin crystallizing in a smaller volume of solvent), filtered through Celite, concentrated to a volume of $1 \mathrm{~mL}$, and allowed to crystallize at room temperature overnight. The product was isolated as blue-green crystals after washing with 2 $\times 1 \mathrm{~mL}$ of hexane and drying in vacuo $(80 \mathrm{mg}, 0.027 \mathrm{mmol}, 72 \%$ yield $)$. X-ray quality crystals of 3-OCN were grown from the slow evaporation of a solution of hexane/ether $\left(2: 1\right.$ ratio) at $-40{ }^{\circ} \mathrm{C}$. Mp ca. $238{ }^{\circ} \mathrm{C}$ (decomp.); IR: $v(\mathrm{OCN}) 2200$ (vs) $\mathrm{cm}^{-1}$. NMR data for 3-OCN was not acquired, as this complex is insoluble in all solvents tested, as observed for 3-N $\mathbf{N}_{3}{ }^{5}$ Anal. Calcd. (\%) for $\mathrm{C}_{140} \mathrm{H}_{232} \mathrm{~N}_{4} \mathrm{O}_{4} \mathrm{U}_{4}(3-\mathrm{OCN}): \mathrm{C}, 56.28 ; \mathrm{H}, 7.83 ; \mathrm{N}, 1.88$. Found: C, 56.60; H, 7.95; N, 1.73.

\section{Generation of Crude $\left(\mathrm{Cp}^{\mathrm{iPr} \mathbf{4}}\right)_{2} \mathrm{U}(\mathrm{F})\left[\left(\boldsymbol{\mu}-\boldsymbol{\eta}^{\mathbf{1}}: \boldsymbol{\eta}^{\mathbf{1}}-\mathrm{OCN}\right) \mathrm{B}\left(\mathrm{C}_{\mathbf{6}} \mathrm{F}_{5}\right)_{3}\right](\mathbf{4}-\mathrm{OCN})$}

A solution of $\mathrm{B}\left(\mathrm{C}_{6} \mathrm{~F}_{5}\right)_{3}(18 \mathrm{mg}, 0.036 \mathrm{mmol}, 4.0$ equiv) in toluene $(1 \mathrm{~mL})$ was added dropwise to a suspension of $\mathbf{3}$ (27 mg, $0.089 \mathrm{mmol}, 1.0$ equiv) in toluene ( $1 \mathrm{~mL})$, and the resulting suspension was stirred for $1 \mathrm{~h}$ at room temperature. During this time, the reaction mixture changed from a green suspension to a clear, dark red-orange solution. Volatiles were removed in vacuo, and the products were extracted into $2 \mathrm{~mL}$ of pentane and filtered through Celite. Volatiles were again removed in vacuo, and the resulting brown residue was extracted with a 1:1 pentane/HMDSO solution ( $0.5 \mathrm{~mL}$ of each), filtered through Celite, concentrated to a volume of $0.5 \mathrm{~mL}$, and cooled to $-40{ }^{\circ} \mathrm{C}$, resulting in the formation of X-ray quality crystals of 4-OCN and, likely, other unidentified products. The IR spectrum of the crystallized material showed strong cyanate/isocyanate stretches at 2358 and $2213 \mathrm{~cm}^{-1}$. 


\section{Generation of Crude $\left(C^{i \mathrm{Pr} 4}\right)_{2} \mathrm{U}(\mathrm{F})\left[\left(\mu-\eta^{\mathbf{1}}: \eta^{\mathbf{1}}-\mathrm{N}_{3}\right) \mathrm{B}\left(\mathrm{C}_{6} \mathrm{~F}_{5}\right)_{3}\right]\left(\mathbf{4}-\mathrm{N}_{3}\right)$}

A solution of $\mathrm{B}\left(\mathrm{C}_{6} \mathrm{~F}_{5}\right)_{3}(11 \mathrm{mg}, 0.022 \mathrm{mmol}, 1.0$ equiv) in toluene (1 mL) was added dropwise to a solution of $\left(\mathrm{Cp}^{\mathrm{iPr} 4}\right)_{2} \mathrm{U}\left(\mathrm{N}_{3}\right)(\mathrm{OTf})(20 \mathrm{mg}, 0.022 \mathrm{mmol}, 1.0$ equiv) in toluene $(1 \mathrm{~mL})$, and the resulting solution was stirred for $30 \mathrm{~min}$ at room temperature. $\mathrm{Me}_{3} \mathrm{SnF}$ (4.2 $\mathrm{mg}, 0.023 \mathrm{mmol}, 1.0$ equiv) was then added as a solid, and the reaction mixture was stirred vigorously for 10 minutes, at which point no visible solids remained. Volatiles were removed in vacuo, and the products were extracted into $2 \mathrm{~mL}$ of pentane, filtered through Celite, concentrated, and allowed to sit undisturbed at room temperature for $24 \mathrm{~h}$. Crystalline material $(26 \mathrm{mg}$ ) was obtained after decanting the pentane solution and drying in vacuo. The ${ }^{19} \mathrm{~F}$ NMR spectrum of the crystallized product revealed a roughly 1.5:1 mixture of $\mathbf{2}-\mathrm{N}_{3}$ and $\mathbf{4}-\mathrm{N}_{3}$ (Figure S14). The IR spectrum of the crystallized product showed strong azide stretches at 2172 and $2094 \mathrm{~cm}^{-1}$. X-ray quality crystals of $\mathbf{4 - N _ { 3 }}$ were grown by slow evaporation of a hexane solution of the crude mixture at room temperature. 


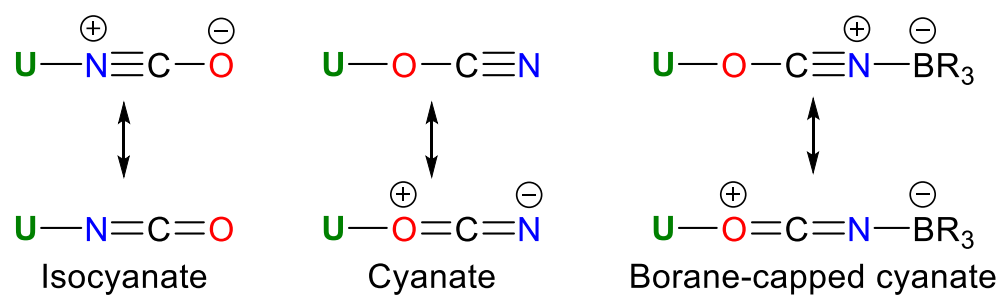

Figure S1. Resonance forms for metal-isocyanate, metal-cyanate, and borane-capped metalcyanate fragments. The resonance form with a $\mathrm{C}-\mathrm{N}$ triple bond is more greatly favored upon coordination of $\mathrm{B}\left(\mathrm{C}_{6} \mathrm{~F}_{5}\right)_{3}$, as illustrated by the isocyanate/cyanate $\mathrm{C}-\mathrm{N}$ stretching frequencies in 2 -

NCO.

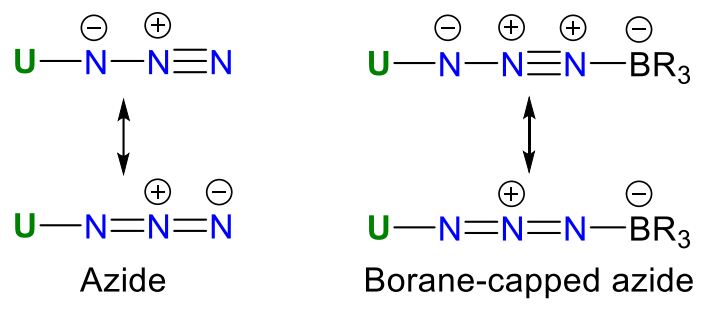

Figure S2. Resonance forms for metal-azide and borane-capped metal-azide fragments.

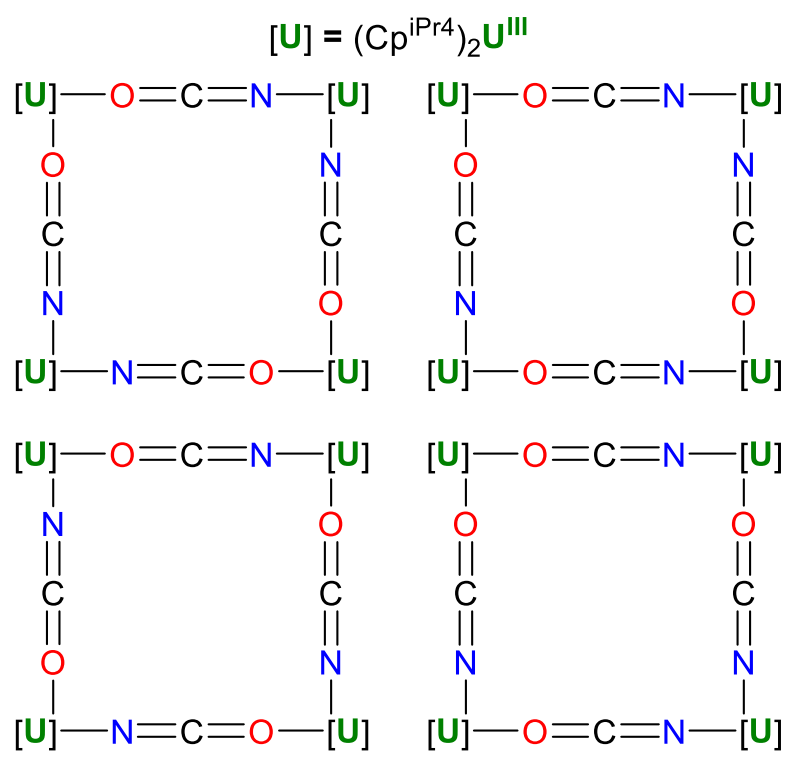

Figure S3. The four possible structural isomers of 3-OCN.

The IR spectrum of 3-OCN shows only one pseudo-asymmetric stretching band. If two isomers were present, they would likely display slightly different values for this stretching frequency. Furthermore, considering the 4 possible isomers above, ignoring the $\mathrm{Cp}^{\mathrm{iPr} 4}$ ligands, these arrangements all have different symmetries (top left: $D_{2 \mathrm{~h}}$, top right: $C_{\mathrm{s}}$, bottom left: $C_{4 \mathrm{~h}}$, bottom 
right: $C_{2 \mathrm{v}}$ ). Only the $C_{4 \mathrm{~h}}$ arrangement (bottom left) would be expected to have exactly one IRactive pseudo-asymmetric stretching band (including the $\mathrm{Cp}^{\mathrm{iPr} 4}$ ligands in this consideration would decrease the symmetry, which generally either does not affect or increases the expected number of bands). Therefore, the IR data are consistent with the bottom left isomer being the only isomer present. However, in the absence of corroborating evidence (see caption for Figure S16), we are hesitant to state this as a definitive assignment, although the bottom left isomer would also be expected based on the assumption that $\left(\mathrm{Cp}^{\mathrm{iPr} 4}\right)_{2} \mathrm{U}(\mathrm{NCO})$ monomers tetramerized to form 3-OCN without any change in isocyanate ligand orientation (i.e. all isocyanate ligands in the monomers were N-bound before tetramerizing with only the formation of new bridging interactions from the O-atom leading to formation of 3-OCN). The isomer is therefore the one depicted in Schemes 3, S2, and S3.

Scheme S1. Synthesis of 1-N3-OTf by Heating a 1:1 Mixture of 1-N3 and 1-OTf.

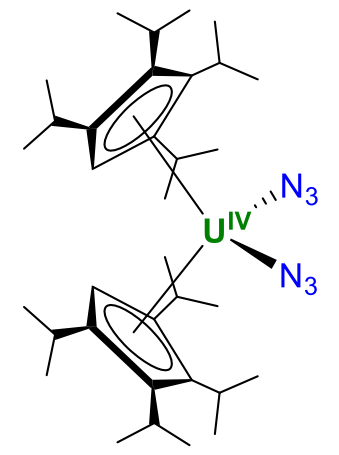

$\left(1-\mathrm{N}_{3}\right)$

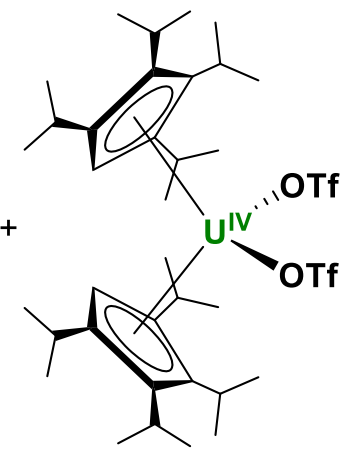

(1-OTf)

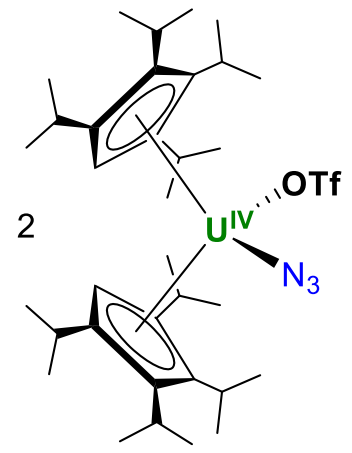

$\left(1-\mathrm{N}_{3}-\mathrm{OTf}\right)$

Scheme S2. Reaction Used to Generate $\left(\mathrm{Cp}^{\mathrm{iPr} 4}\right)_{2} \mathrm{U}(\mathrm{F})\left[\left(\mu-\eta^{1}: \eta^{1}-\mathrm{OCN}\right) \mathrm{B}\left(\mathrm{C}_{6} \mathrm{~F}_{5}\right)_{3}\right]$ (4-OCN) for Single Crystal X-ray Crystallography

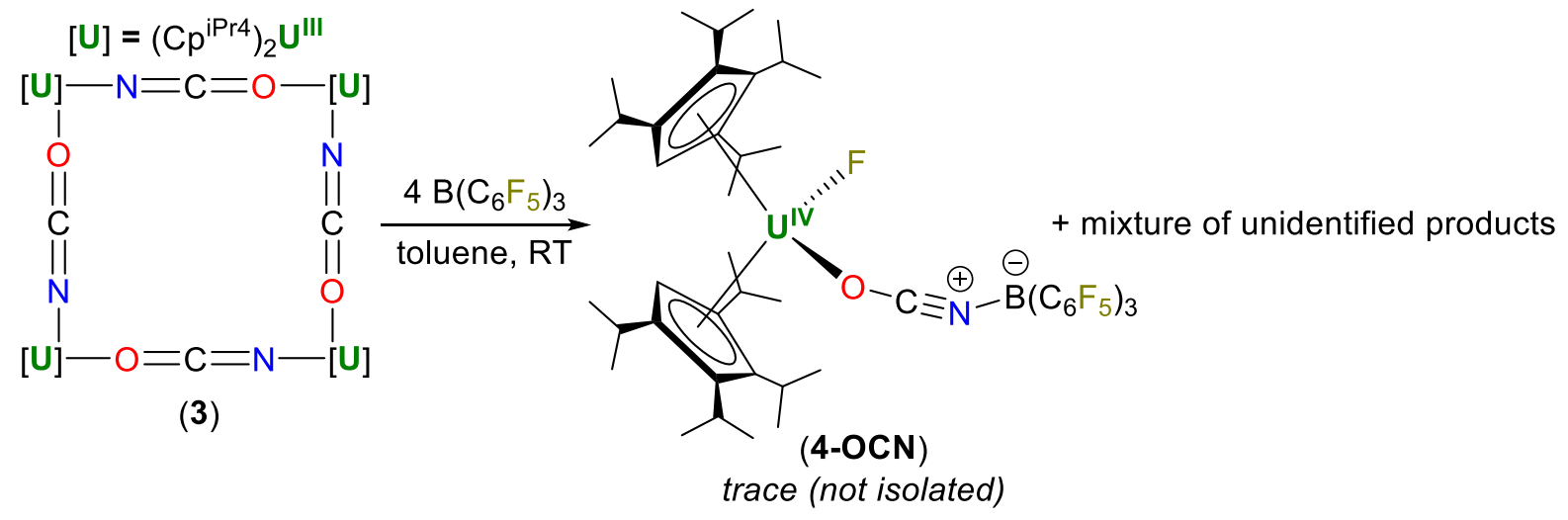


Scheme S3. Proposed Mechanism for the Formation of Trace Quantities of 4-OCN in Scheme S2

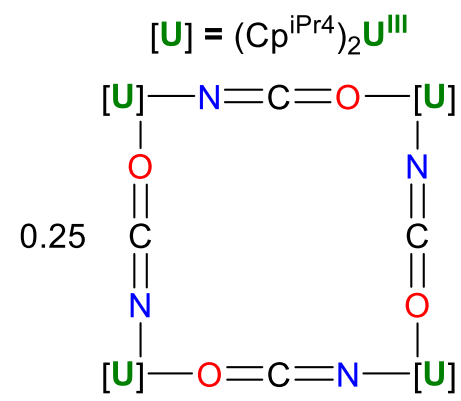

(3-OCN)

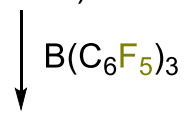

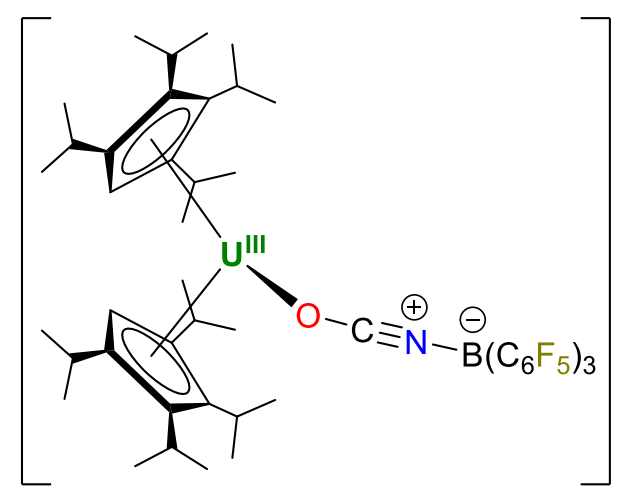

Not observed

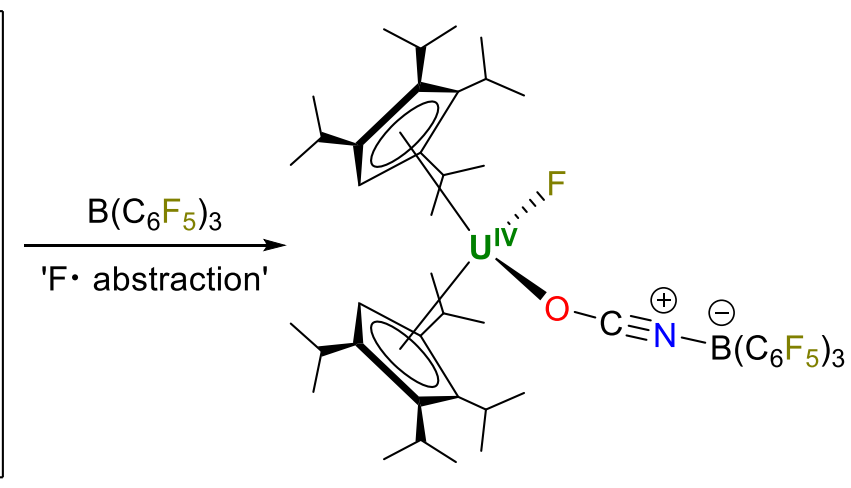

$(4-O C N)$

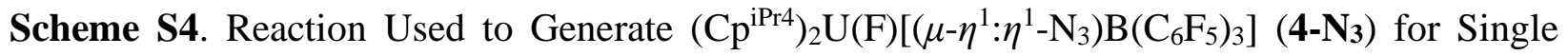
Crystal X-ray Crystallography. ${ }^{\text {a }}$

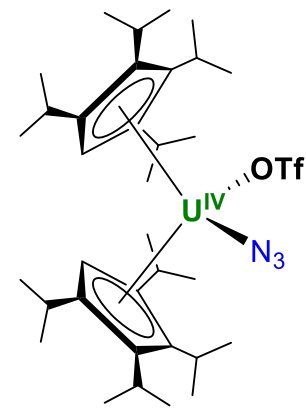

$\left(1-\mathrm{OTf}-\mathrm{N}_{3}\right)$

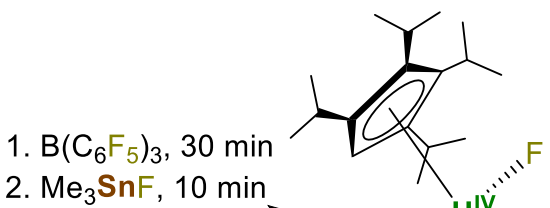

toluene, RT $-\mathrm{Me}_{3} \mathrm{SnOTf}$

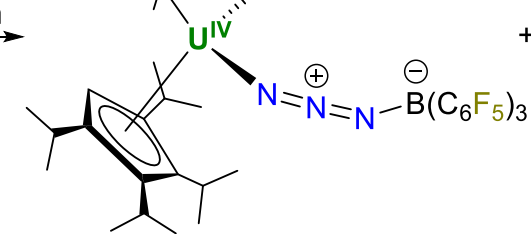

$\left(4-\mathrm{N}_{3}\right)$

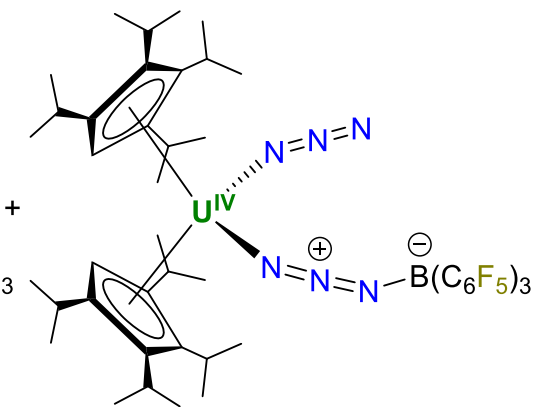

$\left(2-\mathrm{N}_{3}\right)$

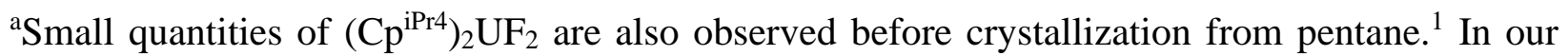
hands, it was not possible to separate the resulting mixture of $\mathbf{4 - N _ { 3 }}$ and $\mathbf{2}-\mathbf{N}_{\mathbf{3}}$. 


\section{NMR Spectroscopy}

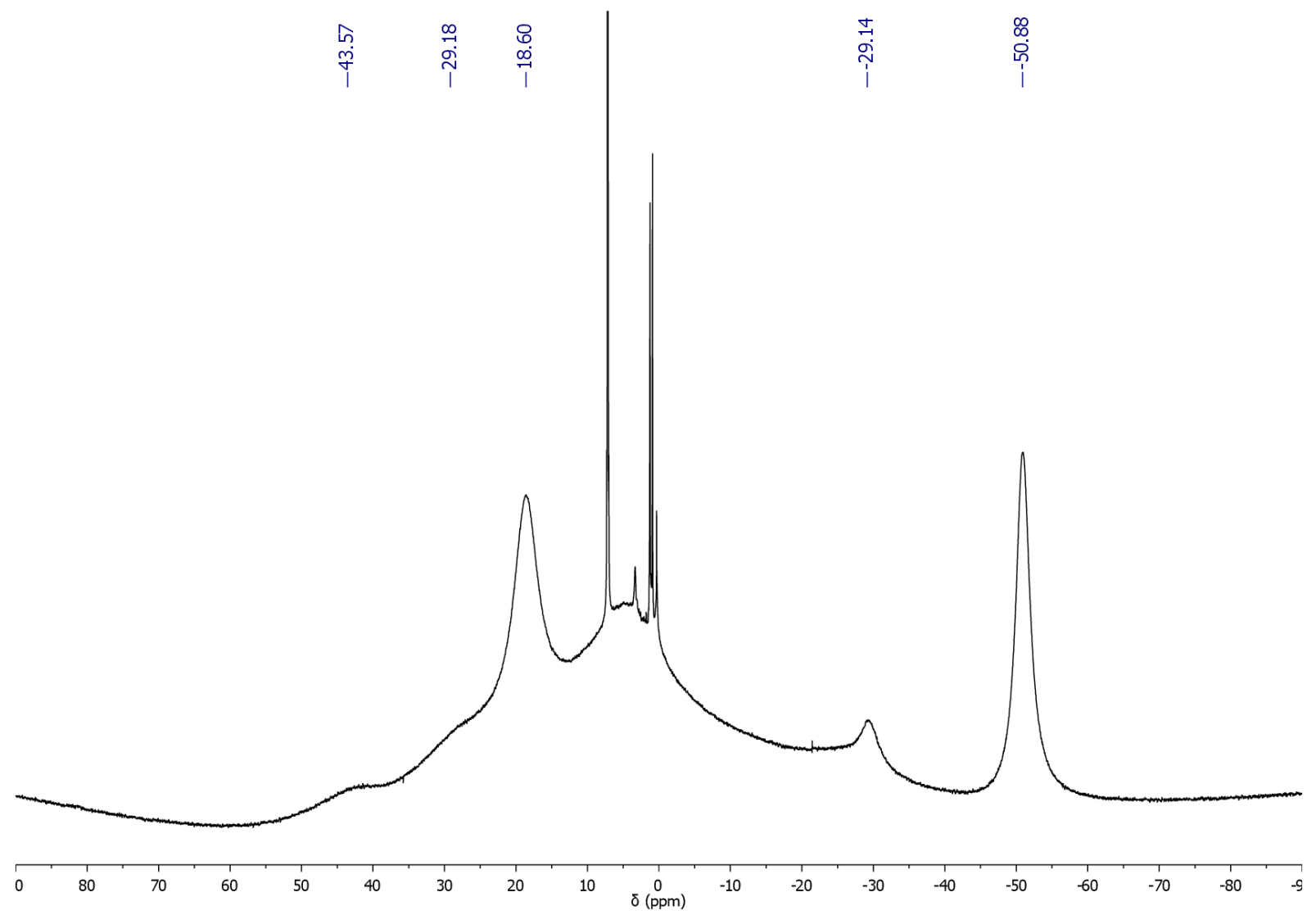

Figure S4. Room temperature ${ }^{1} \mathrm{H}$ NMR spectrum of $\left(\mathrm{Cp}^{\mathrm{iPr} 4}\right)_{2} \mathrm{U}\left(\mathrm{N}_{3}\right)_{2}\left(\mathbf{1}-\mathrm{N}_{\mathbf{3}}\right)$ in $\mathrm{C}_{6} \mathrm{D}_{6}$ with a $10 \mathrm{~Hz}$ exponential line broadening function applied to improve visibility of very broad signals. Due to the extreme broadness of the signals, it was not possible to correct the baseline of this spectrum. The chemical shift values shown are roughly centered on what appear to be individual broad signals, so their placement is tentative. Minor quantities of organic impurities, primarily $n$-hexane, appear as much sharper peaks between 0 and $4 \mathrm{ppm}$ and are thus visible despite low concentrations

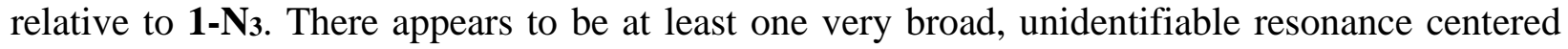
within the diamagnetic region. 


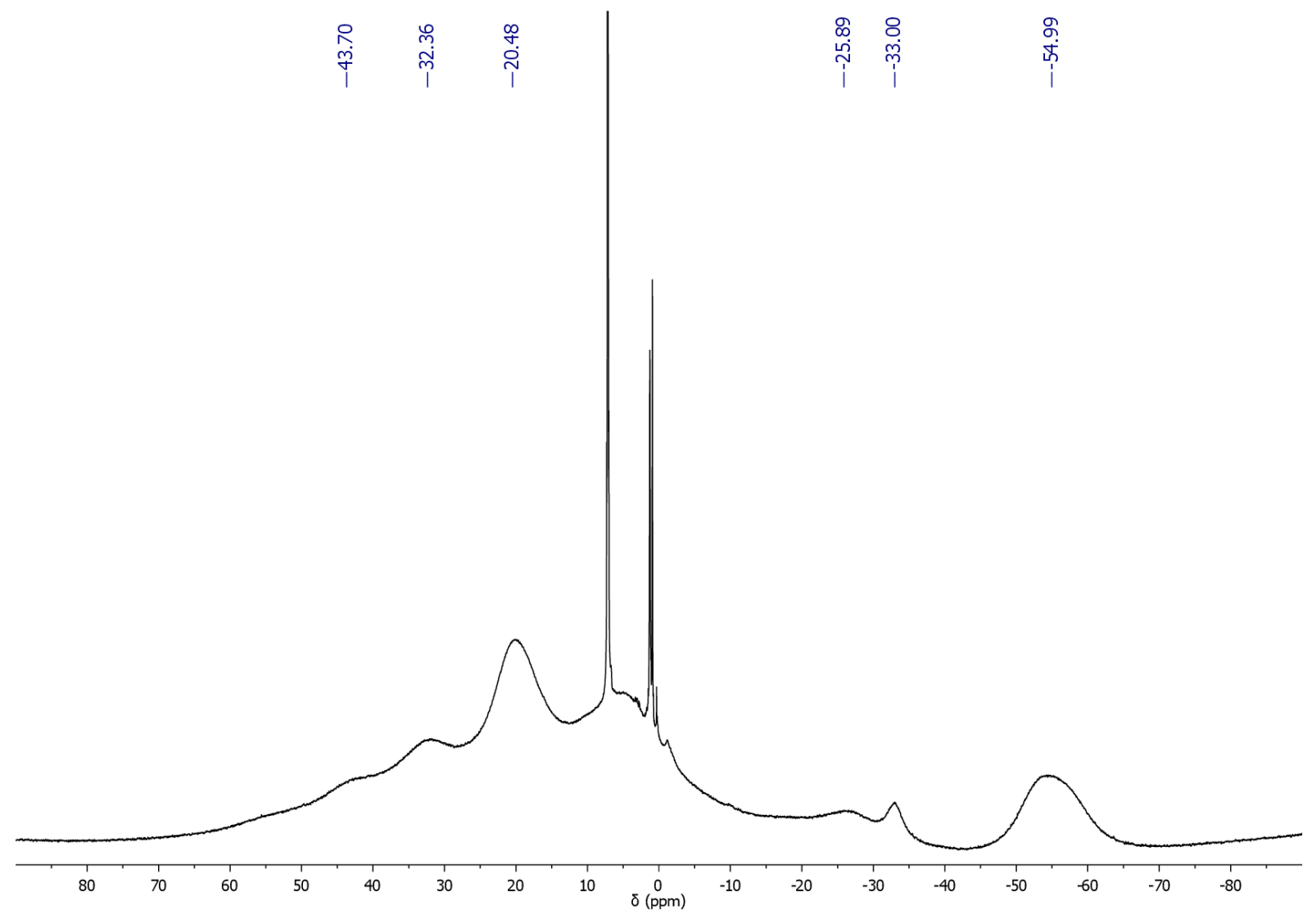

Figure S5. Room temperature ${ }^{1} \mathrm{H}$ NMR spectrum of $\left(\mathrm{Cp}^{\mathrm{iPr} 4}\right)_{2} \mathrm{U}(\mathrm{NCO})_{2}(\mathbf{1 - N C O})$ in $\mathrm{C}_{6} \mathrm{D}_{6}$ with a $10 \mathrm{~Hz}$ exponential line broadening function applied to improve visibility of very broad signals. Due to the extreme broadness of the signals, it was not possible to correct the baseline of this spectrum. The chemical shift values shown are roughly centered on what appear to be individual broad signals, so their placement is tentative. Minor quantities of organic impurities, primarily $n$ hexane, appear as much sharper peaks between 0 and 4 ppm and are thus visible despite low concentrations relative to 1-NCO. There appears to be at least one very broad, unidentifiable resonance centered within the diamagnetic region. 


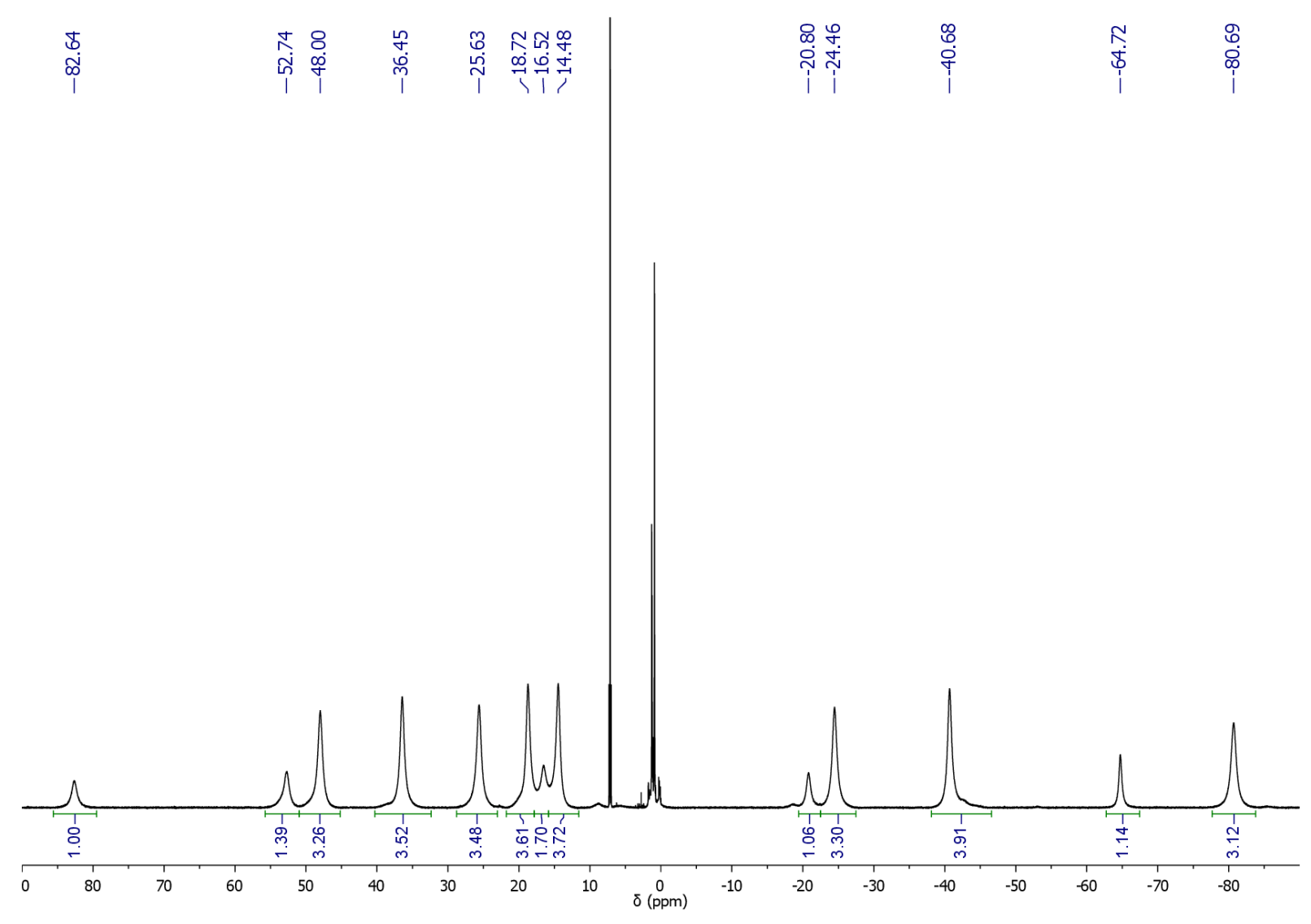

Figure S6. Room temperature ${ }^{1} \mathrm{H}$ NMR spectrum of $\left(\mathrm{Cp}^{\mathrm{iPr} 4}\right)_{2} \mathrm{U}(\mathrm{OTf})_{2}$ (1-OTf) in $\mathrm{C}_{6} \mathrm{D}_{6}$. Minor quantities of organic impurities, primarily $n$-hexane, appear as much sharper peaks between 0 and $4 \mathrm{ppm}$ and are thus visible despite low concentrations relative to 1-OTf. 


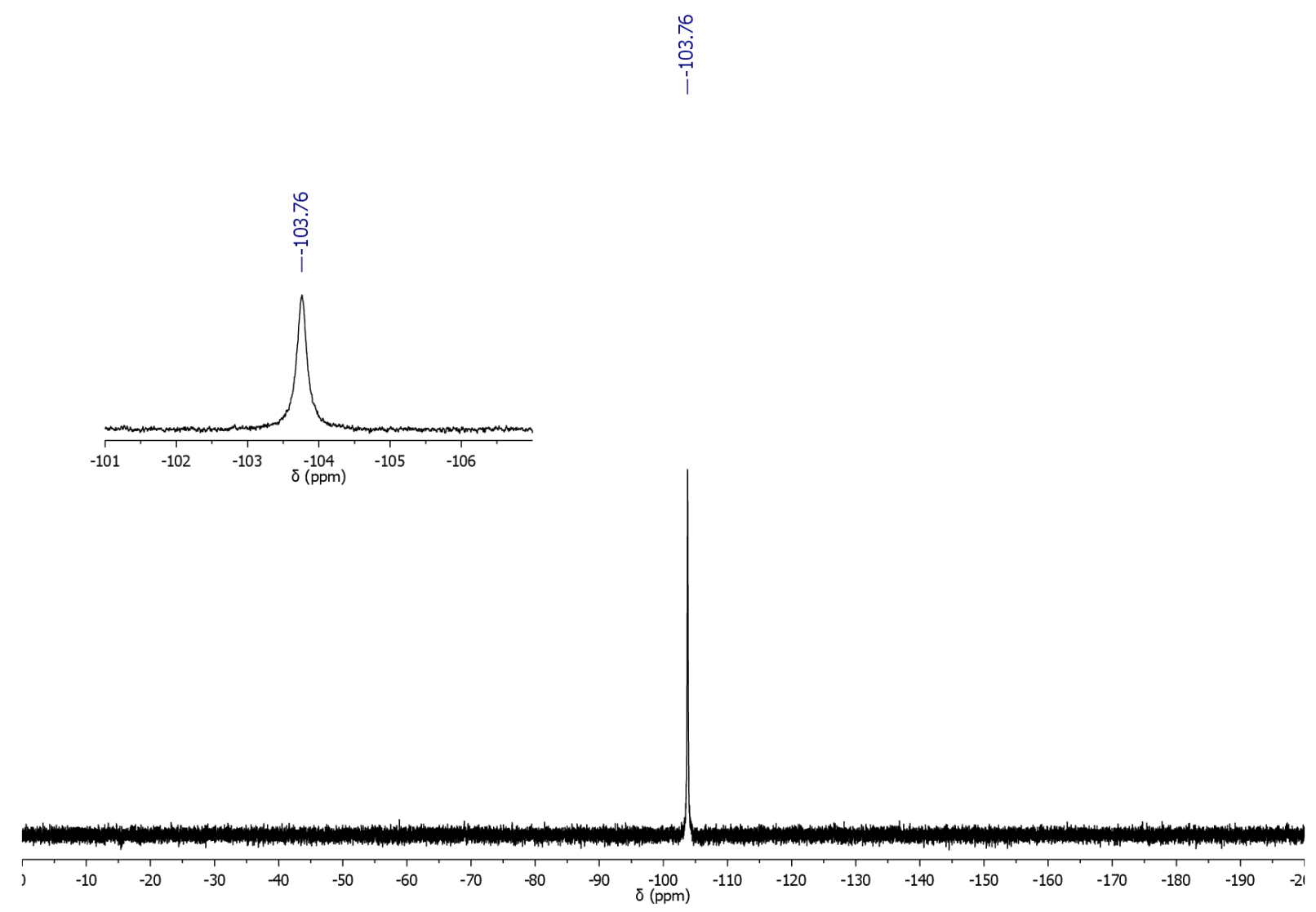

Figure S7. Room temperature ${ }^{19} \mathrm{~F}$ NMR spectrum of $\left(\mathrm{Cp}^{\mathrm{iPr} 4}\right)_{2} \mathrm{U}(\mathrm{OTf})_{2}(\mathbf{1 - O T f})$ in $\mathrm{C}_{6} \mathrm{D}_{6}$. 


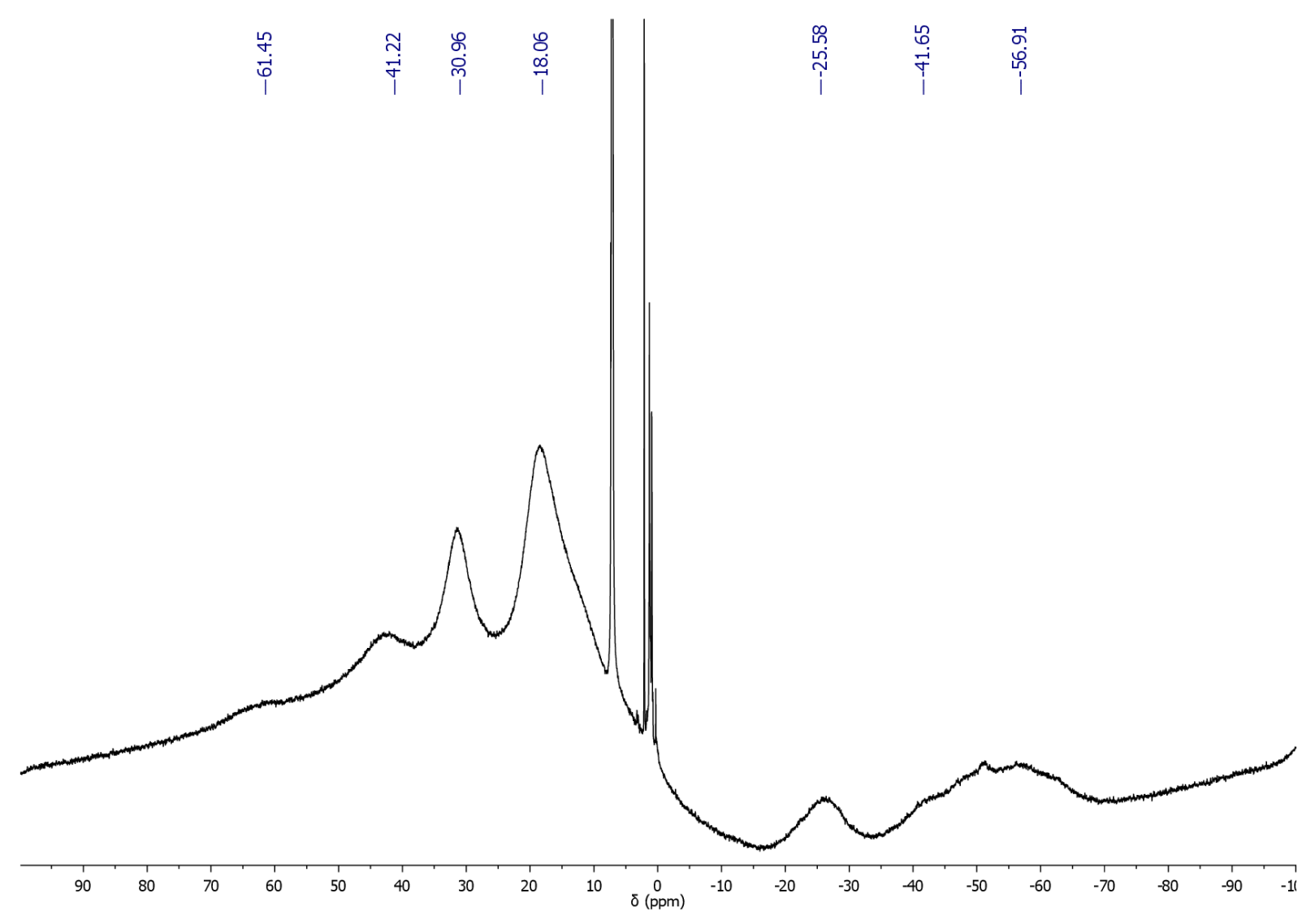

Figure S8. Room temperature ${ }^{1} \mathrm{H}$ NMR spectrum of $\left(\mathrm{Cp}^{\mathrm{iPr} 4}\right)_{2} \mathrm{U}\left(\mathrm{N}_{3}\right)(\mathrm{OTf})(\mathbf{1 - N} 3-\mathbf{O T f})$ in $\mathrm{C}_{6} \mathrm{D}_{6}$ with a $10 \mathrm{~Hz}$ exponential line broadening function applied to improve visibility of very broad signals. Due to the extreme broadness of the signals, it was not possible to correct the baseline of this spectrum. The chemical shift values shown are roughly centered on what appear to be individual broad signals, so their placement is tentative. Minor quantities of organic impurities, primarily $n$ hexane and toluene, appear as much sharper peaks between 0 and $7 \mathrm{ppm}$ and are thus visible despite low concentrations relative to 1-N3-OTf. Additionally, a very small signal attributed to trace quantities of $\left(\mathrm{Cp}^{\mathrm{iPr} 4}\right)_{2} \mathrm{U}\left(\mathrm{N}_{3}\right)_{2}\left(\mathbf{1}-\mathrm{N}_{3}\right)$ is visible at ca. $-50.9 \mathrm{ppm}$ (see Figure $\mathrm{S} 4$ ). 


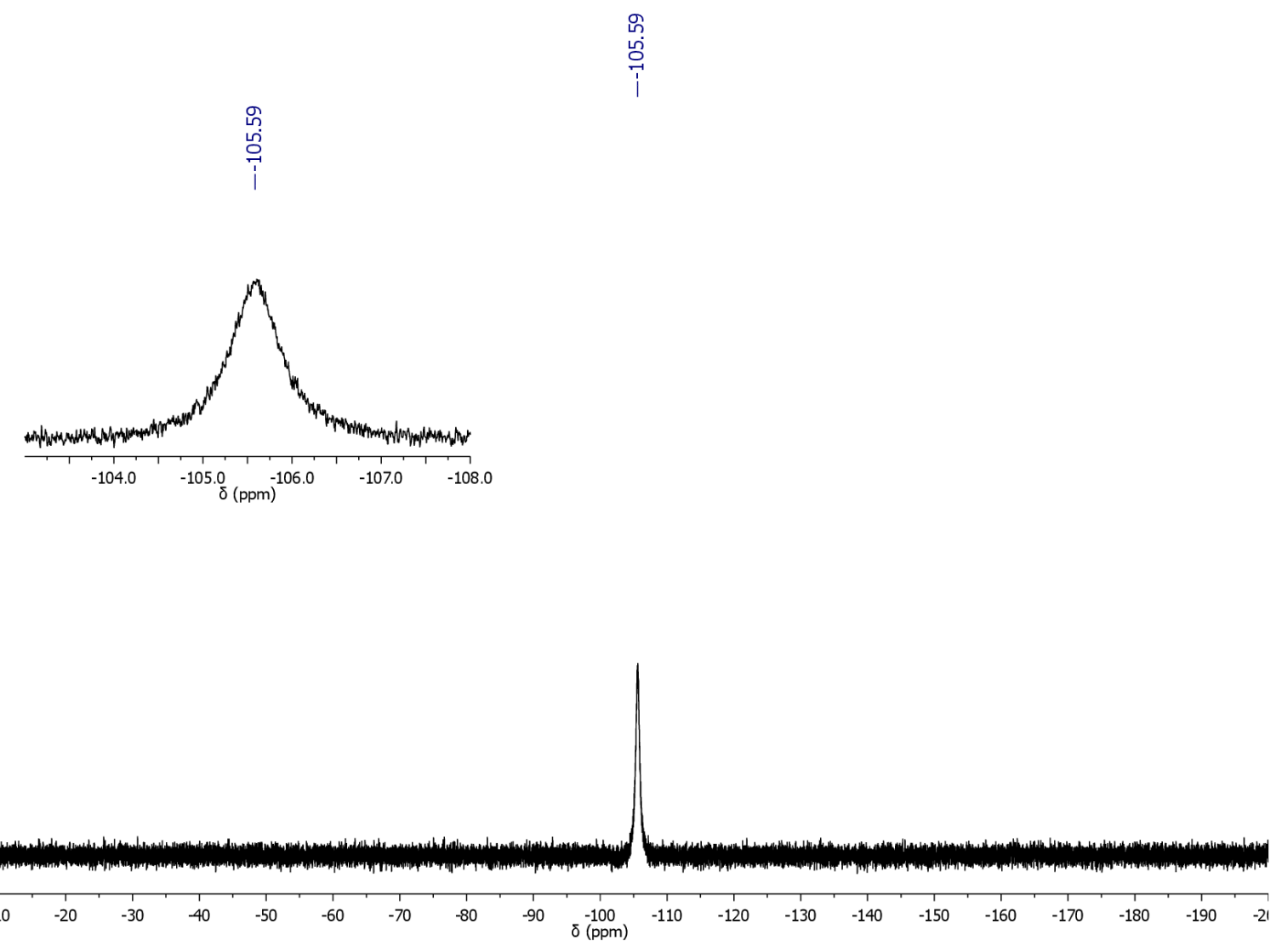

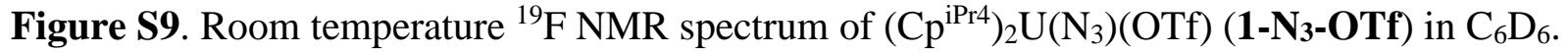




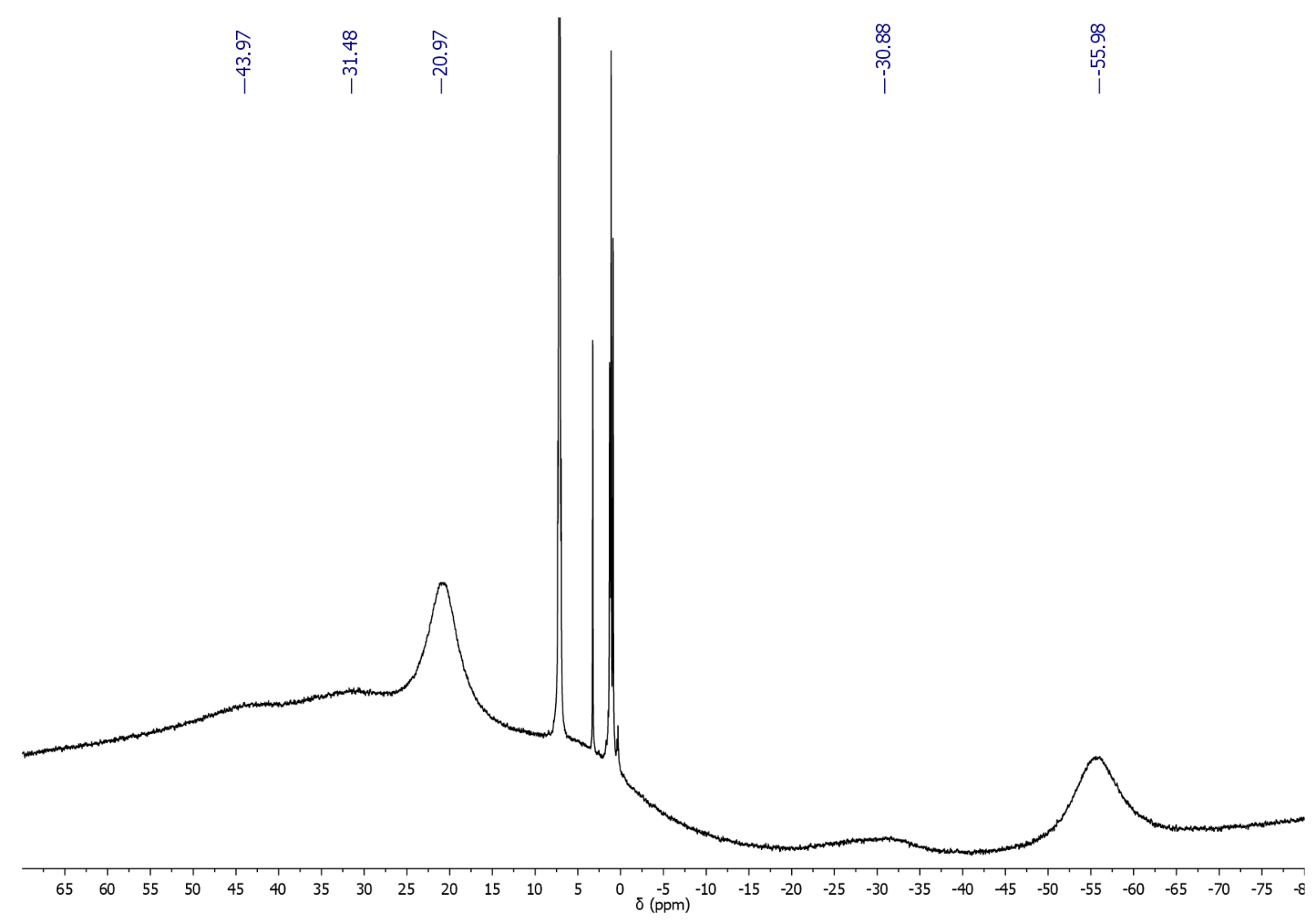

Figure S10. Room temperature ${ }^{1} \mathrm{H}$ NMR spectrum of $\left(\mathrm{Cp}^{\mathrm{iPr} 4}\right)_{2} \mathrm{U}\left(\mathrm{N}_{3}\right)\left[\left(\mu-\eta^{1}: \eta^{1}-\mathrm{N}_{3}\right) \mathrm{B}\left(\mathrm{C}_{6} \mathrm{~F}_{5}\right)_{3}\right]\left(\mathbf{2}-\mathrm{N}_{3}\right)$ in $\mathrm{C}_{6} \mathrm{D}_{6}$ with a $10 \mathrm{~Hz}$ exponential line broadening function applied to improve visibility of very broad signals. Due to the extreme broadness of the signals, it was not possible to correct the baseline of this spectrum. The chemical shift values shown are roughly centered on what appear to be individual broad signals, so their placement is tentative. Minor quantities of organic impurities, primarily diethyl ether and $n$-hexane, appear as much sharper peaks between 0 and 4 ppm and are thus visible despite low concentrations relative to $\mathbf{2}-\mathbf{N}_{\mathbf{3}}$. 


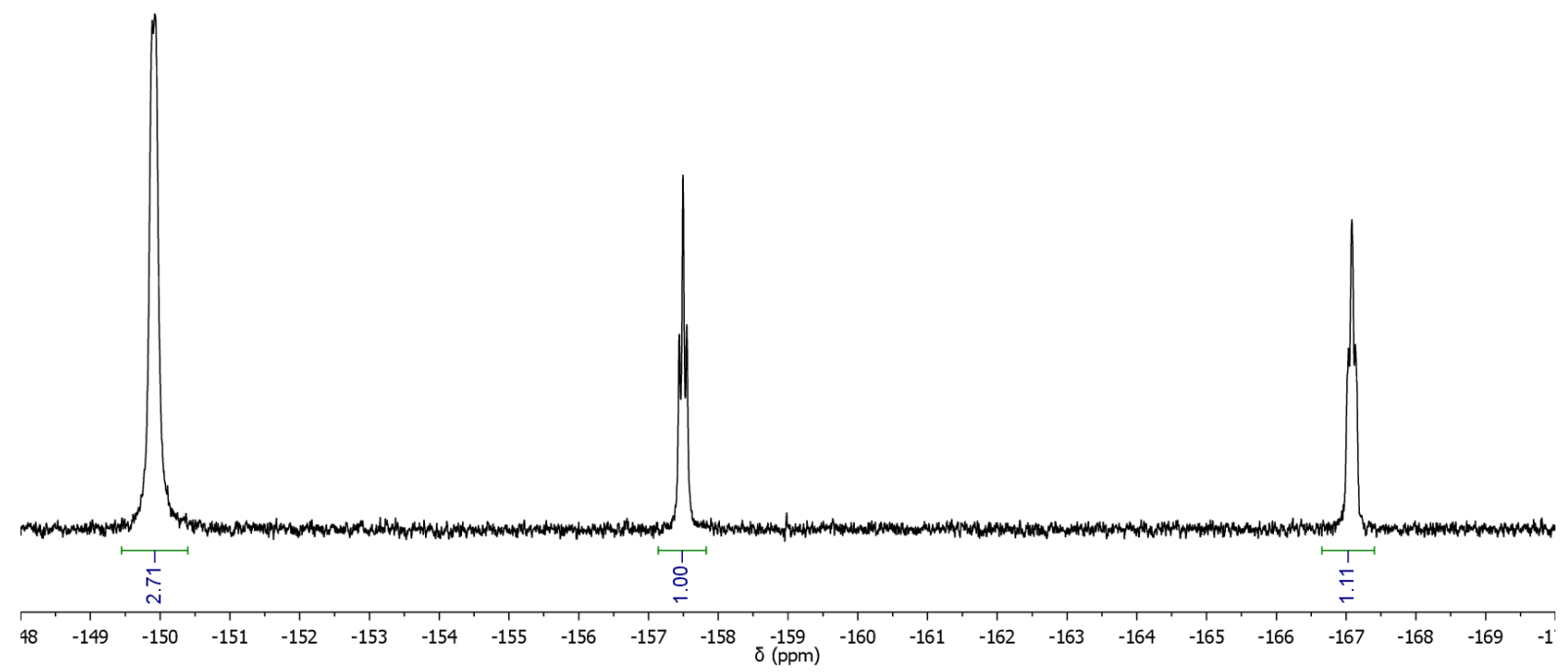

Figure S11. Room temperature ${ }^{19} \mathrm{~F}$ NMR spectrum of $\left(\mathrm{Cp}^{\mathrm{iPr} 4}\right)_{2} \mathrm{U}_{\left(\mathrm{N}_{3}\right)}\left[\left(\mu-\eta^{1}: \eta^{1}-\mathrm{N}_{3}\right) \mathrm{B}\left(\mathrm{C}_{6} \mathrm{~F}_{5}\right)_{3}\right]\left(2-\mathrm{N}_{3}\right)$ in $\mathrm{C}_{6} \mathrm{D}_{6}$. 


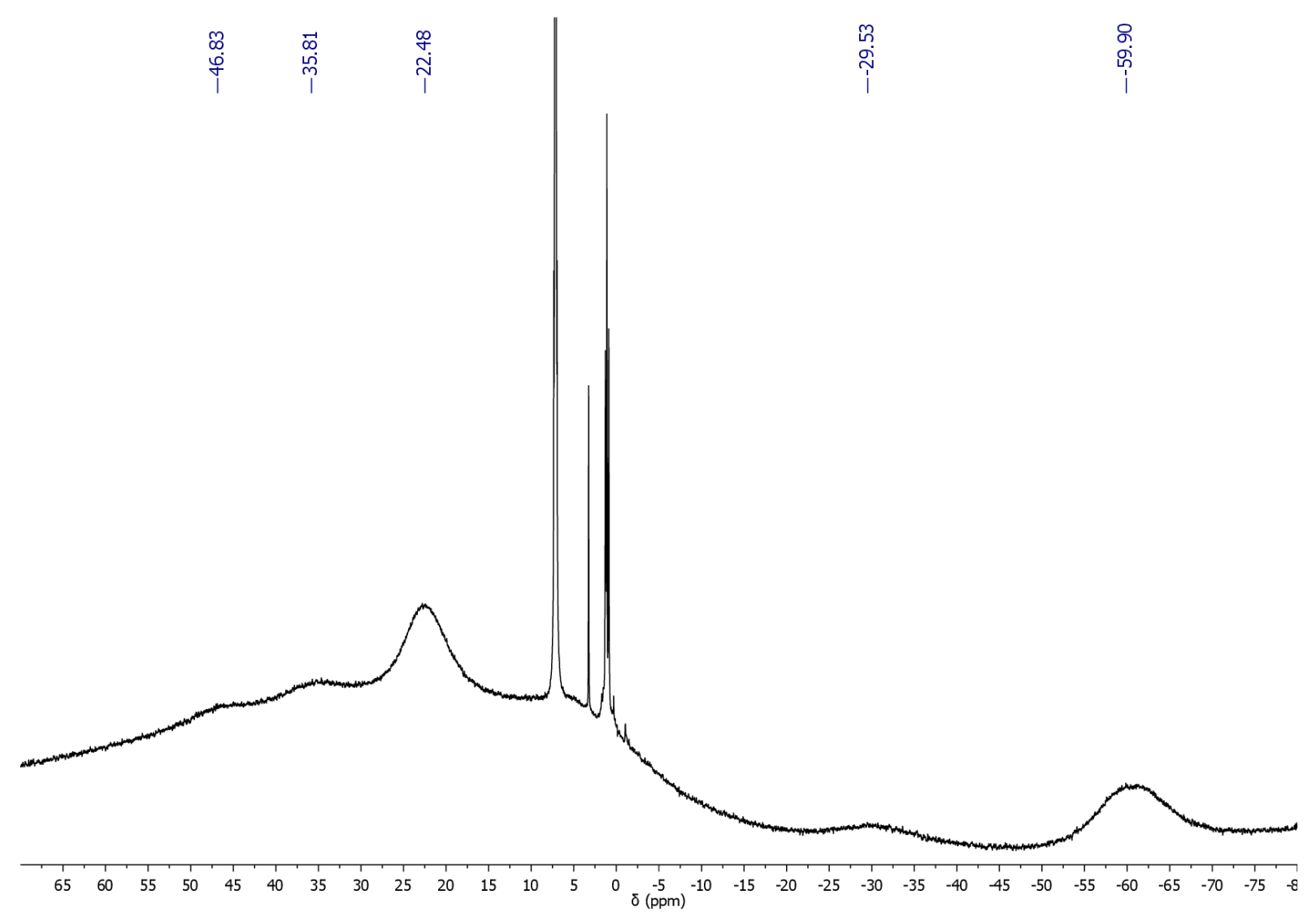

Figure S12. Room temperature ${ }^{1} \mathrm{H}$ NMR spectrum of $\left(\mathrm{Cp}^{\mathrm{iPr} 4}\right)_{2} \mathrm{U}(\mathrm{NCO})\left[\left(\mu-\eta^{1}: \eta^{1}-\mathrm{OCN}\right) \mathrm{B}\left(\mathrm{C}_{6} \mathrm{~F}_{5}\right)_{3}\right]$ (2-NCO) in $\mathrm{C}_{6} \mathrm{D}_{6}$ with a $10 \mathrm{~Hz}$ exponential line broadening function applied to improve visibility of very broad signals. Due to the extreme broadness of the signals, it was not possible to correct the baseline of this spectrum. The chemical shift values shown are roughly centered on what appear to be individual broad signals, so their placement is tentative. Minor quantities of organic impurities, primarily diethyl ether and $n$-hexane, appear as much sharper peaks between 0 and 4 ppm and are thus visible despite low concentrations relative to 2-NCO. 


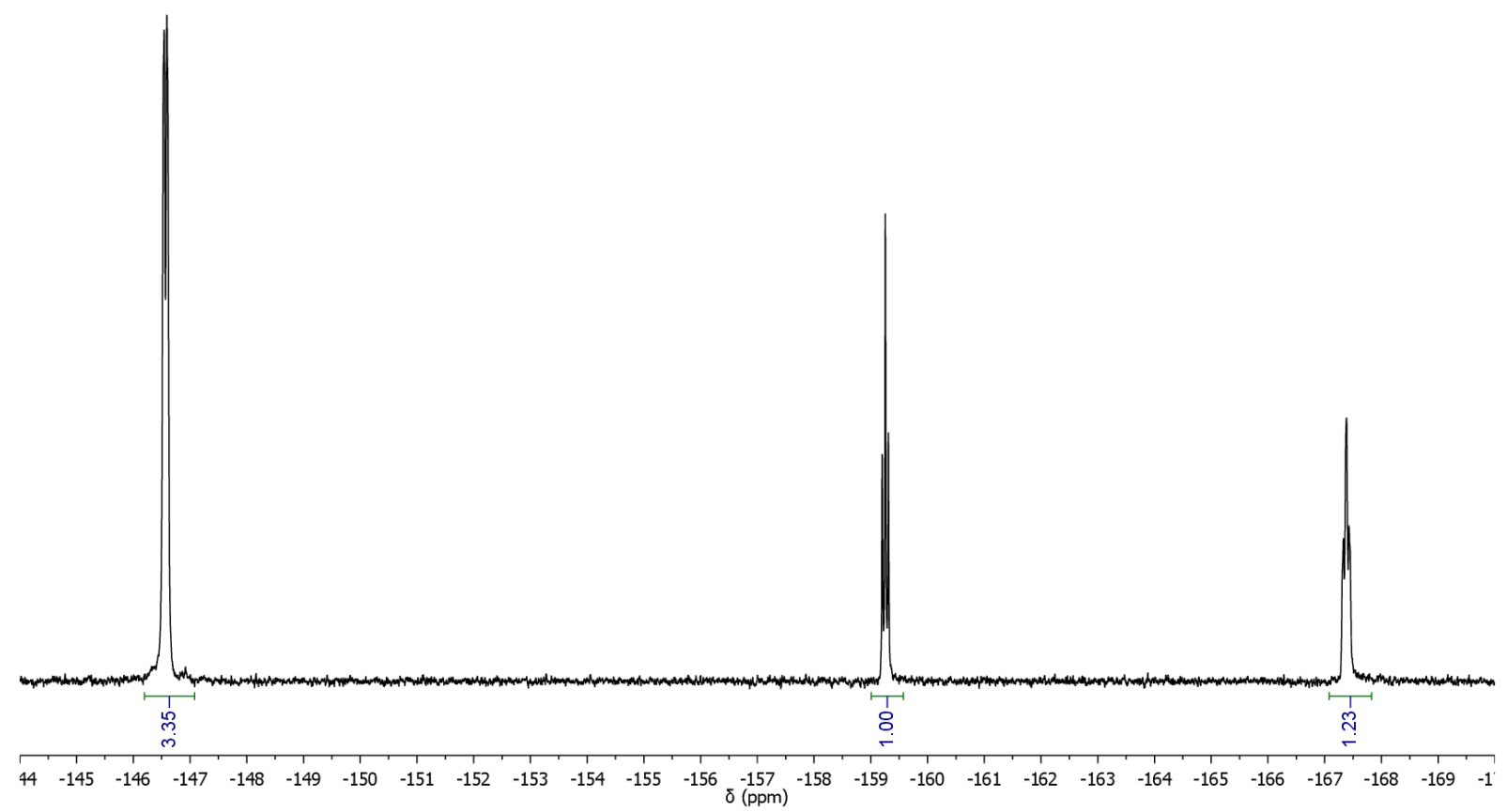

Figure S13. Room temperature ${ }^{19} \mathrm{~F}$ NMR spectrum of $\left(\mathrm{Cp}^{\mathrm{iPr} 4}\right)_{2} \mathrm{U}(\mathrm{NCO})\left[\left(\mu-\eta^{1}: \eta^{1}-\mathrm{OCN}\right) \mathrm{B}\left(\mathrm{C}_{6} \mathrm{~F}_{5}\right)_{3}\right]$ (2-NCO) in $\mathrm{C}_{6} \mathrm{D}_{6}$. 


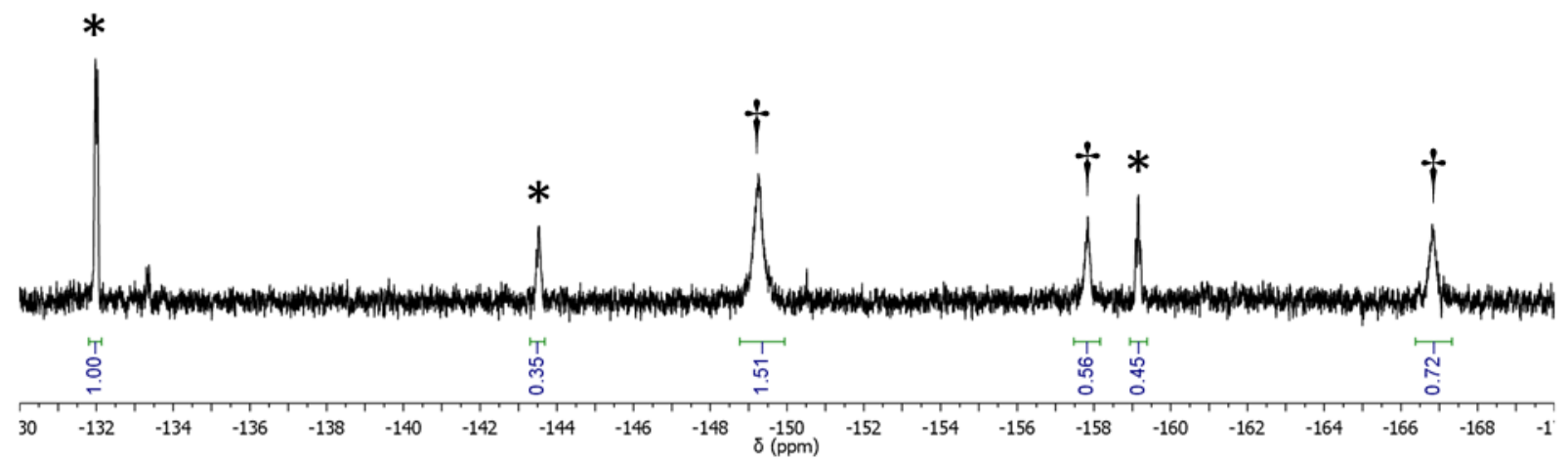

Figure S14. Room temperature ${ }^{19}$ F NMR spectrum of the crystallized mixture of 4-N3 (*) and 2$\mathbf{N}_{3}(\dagger)$ (see Experimental Section and Scheme S4). 


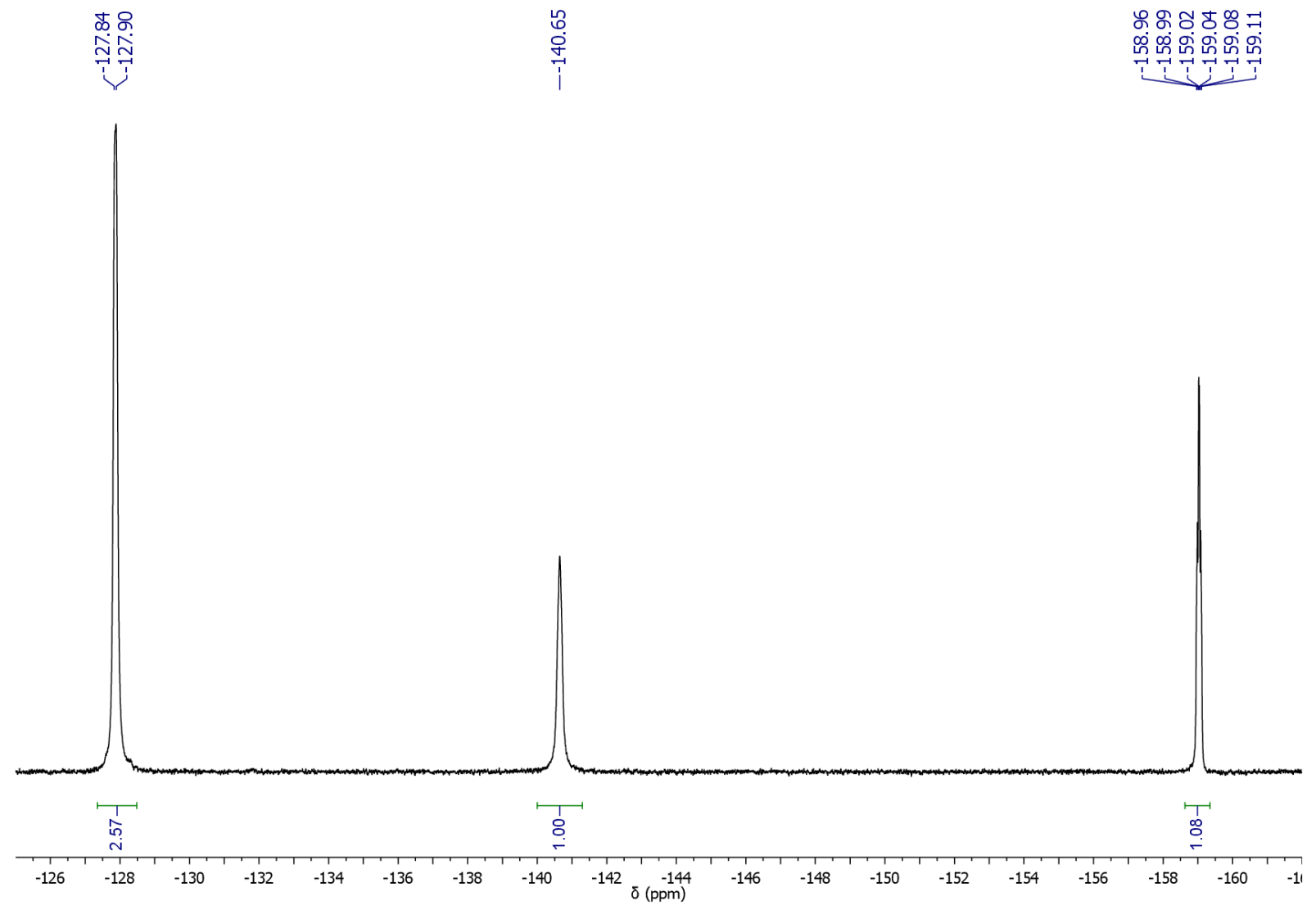

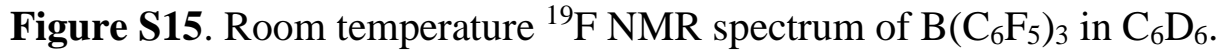




\section{X-ray Crystallography}

In a dry nitrogen glovebox, samples of single crystals of 1-N 3 , 1-NCO, 1-OTf, 1-N3-OTf, 2-N 2-NCO, 3-OCN, 4-OCN, and 4-N3 were coated in Paratone-N oil prior to transport to diffraction facilities, where they were evaluated by polarized light microscopy and mounted on a Kaptan loop (for 1-N3, $\mathbf{1 - N C O}$, and 1-N3-OTf) or on a MiTeGen $10 \mu \mathrm{m}$ aperture Dual-Thickness MicroMount (for 1-OTf, 2-N3, 2-NCO, 3-OCN, 4-OCN, and 4-N3). X-ray diffraction data for 1-N3, 1-NCO, and 1-N3-OTf were collected at CheXray, Berkeley, CA, using a Bruker APEX II QUAZAR instrument outfitted with a monochromated radiation source (for 1-N 3 and 1-N3-OTf) or on a Rigaku XtaLAB P200 equipped with a MicroMax-007 HF microfocus rotating anode and a Pilatus 200K hybrid pixel array detector (for 1-NCO); data for these samples were collected using Mo K $\alpha$ radiation $(\lambda=0.71073 \AA$ ). X-ray diffraction data for 1-OTf, 2-N3, 2-NCO, 3-OCN, 4-OCN, and 4-N3 were collected at the Advanced Light Source (ALS), Lawrence Berkeley National Lab, Berkeley, CA, station 12.2.1 using a silicon monochromated beam of $17 \mathrm{keV}(\lambda=0.7288 \AA)$ synchrotron radiation. All data collections were conducted at $100 \mathrm{~K}$, with the crystals cooled by a stream of dry nitrogen. For 1-N 3 , 1-OTf, 1-N3-OTf, 2-N 3 , 2-NCO, 3-OCN, 4-OCN, and 4-N3 Bruker APEX2 or APEX3 software was used for the data collections, Bruker SAINT V8.37A or V8.38A software was used to conduct the cell refinement and data reduction procedures, ${ }^{6}$ and absorption corrections were carried out by a multi-scan method utilizing the SADABS program. ${ }^{7}$ For 1-NCO, CrysAlis ${ }^{\mathrm{Pro}}$ was used for the data collections and data processing, including a multiscan absorption correction applied using the SCALE3 ABSPACK scaling algorithm within CrysAlis ${ }^{\text {Pro }}{ }^{8}$ Initial structure solutions were found using direct methods (SHELXT), ${ }^{9}$ and refinements were carried out using SHELXL-2014. ${ }^{10-12}$ Thermal parameters for all non-hydrogen atoms were refined anisotropically. Thermal ellipsoid plots were made using Mercury. ${ }^{13}$ Complexes 1-OTf and 3-OCN possessed highly disordered outer sphere solvent molecules; the data for these structures were treated with the SQUEEZE ${ }^{14}$ routine included in PLATON (for 1OTf) or the BYPASS ${ }^{15}$ routine included in the OLEX2 software package (for 3-OCN). All structures have been deposited to the Cambridge Crystallographic Data Centre (CCDC), with deposition numbers 1994710 (1-N3), 1994711 (1-NCO), 1994712 (1-OTf), 1994713 (1-N3-OTf), 1994714 (2-N3), 1994715 (2-NCO), 1994716 (3-OCN), 1994717 (4-OCN), and 1994718 (4-N3). 
Table S1. Selected distances $(\AA)$ and angles $\left({ }^{\circ}\right)$ for $\mathbf{1}-\mathrm{N}_{3}$ and 1-NCO.

\begin{tabular}{ll|ll}
\multicolumn{2}{c|}{$\mathbf{1 - N}_{\mathbf{3}}$} & \multicolumn{2}{c}{$\mathbf{1 - N C O}$} \\
\hline $\mathrm{U}(1)-\mathrm{N}(1)$ & $2.234(2)$ & $\mathrm{U}(1)-\mathrm{N}(1)$ & $2.271(2)$ \\
$\mathrm{U}(1)-\mathrm{N}(4)$ & $2.242(2)$ & $\mathrm{U}(1)-\mathrm{N}(2)$ & $2.268(3)$ \\
$\mathrm{N}(1)-\mathrm{N}(2)$ & $1.196(3)$ & $\mathrm{N}(1)-\mathrm{C}(1)$ & $1.173(4)$ \\
$\mathrm{N}(2)-\mathrm{N}(3)$ & $1.147(3)$ & $\mathrm{N}(2)-\mathrm{C}(2)$ & $1.156(4)$ \\
$\mathrm{N}(4)-\mathrm{N}(5)$ & $1.187(3)$ & $\mathrm{C}(1)-\mathrm{O}(1)$ & $1.186(4)$ \\
$\mathrm{N}(5)-\mathrm{N}(6)$ & $1.141(4)$ & $\mathrm{C}(2)-\mathrm{O}(2)$ & $1.189(4)$ \\
$\mathrm{U}(1)-\mathrm{N}(1)-\mathrm{N}(2)$ & $173.0(2)$ & $\mathrm{U}(1)-\mathrm{N}(1)-\mathrm{C}(1)$ & $170.6(2)$ \\
$\mathrm{U}(1)-\mathrm{N}(4)-\mathrm{N}(5)$ & $160.8(2)$ & $\mathrm{U}(1)-\mathrm{N}(2)-\mathrm{C}(2)$ & $158.9(3)$ \\
$\mathrm{N}(1)-\mathrm{U}(1)-\mathrm{N}(4)$ & $92.86(8)$ & $\mathrm{N}(1)-\mathrm{U}(1)-\mathrm{N}(2)$ & $93.8(1)$ \\
$\mathrm{Cp}($ cent $)-\mathrm{U}$ & $2.468(1), 2.481(1)$ & $\mathrm{Cp}($ cent $)-\mathrm{U}$ & $2.468(2), 2.480(2)$ \\
$\mathrm{Cp}($ cent $)-\mathrm{U}-\mathrm{Cp}($ cent $)$ & $147.50(4)$ & $\mathrm{Cp}($ cent $)-\mathrm{U}-\mathrm{Cp}($ cent $)$ & $147.48(5)$
\end{tabular}

Table S2. Selected distances $(\AA)$ and angles $\left(^{\circ}\right)$ for 1-N3-OTf and 1-OTf.

\begin{tabular}{ll|ll}
\multicolumn{2}{c|}{ 1-N3-OTf } & \multicolumn{2}{|c}{ 1-OTf } \\
\hline $\mathrm{U}(1)-\mathrm{N}(1)$ & $2.215(6)$ & $\mathrm{U}(1)-\mathrm{O}(1)$ & $2.289(6)$ \\
$\mathrm{U}(1)-\mathrm{O}(1)$ & $2.318(4)$ & $\mathrm{U}(1)-\mathrm{O}(4)$ & $2.302(6)$ \\
$\mathrm{N}(1)-\mathrm{N}(2)$ & $1.198(8)$ & - & - \\
$\mathrm{N}(2)-\mathrm{N}(3)$ & $1.171(8)$ & - & - \\
$\mathrm{U}(1)-\mathrm{N}(1)-\mathrm{N}(2)$ & $158.7(4)$ & $\mathrm{U}(1)-\mathrm{O}(1)-\mathrm{S}(1)$ & $162.3(4)$ \\
$\mathrm{U}(1)-\mathrm{O}(1)-\mathrm{S}(1)$ & $175.4(3)$ & $\mathrm{U}(1)-\mathrm{O}(4)-\mathrm{S}(2)$ & $167.2(4)$ \\
$\mathrm{N}(1)-\mathrm{U}(1)-\mathrm{O}(1)$ & $96.7(2)$ & $\mathrm{O}(1)-\mathrm{U}(1)-\mathrm{O}(4)$ & $85.7(2)$ \\
$\mathrm{Cp}($ cent $)-\mathrm{U}$ & $2.453(3), 2.479(3)$ & $\mathrm{Cp}($ cent $)-\mathrm{U}$ & $2.466(3), 2.475(4)$ \\
$\mathrm{Cp}($ cent $)-\mathrm{U}-\mathrm{Cp}($ cent $)$ & $141.33(9)$ & $\mathrm{Cp}($ cent $)-\mathrm{U}-\mathrm{Cp}($ cent $)$ & $135.3(1)$
\end{tabular}


Table S3. Selected distances $(\AA)$ and angles $\left({ }^{\circ}\right)$ for $\mathbf{2}-\mathrm{N}_{3}$ and $\mathbf{2}$-NCO.

\begin{tabular}{ll|lr}
\multicolumn{2}{c|}{$2-N_{3}$} & \multicolumn{2}{c}{ 2-NCO } \\
\hline $\mathrm{U}(1)-\mathrm{N}(1)$ & $2.166(3)$ & $\mathrm{U}(1)-\mathrm{N}(1)$ & $2.244(2)$ \\
$\mathrm{U}(1)-\mathrm{N}(4)$ & $2.397(3)$ & $\mathrm{U}(1)-\mathrm{O}(1)$ & $2.323(2)$ \\
$\mathrm{N}(1)-\mathrm{N}(2)$ & $1.191(5)$ & $\mathrm{N}(1)-\mathrm{C}(1)$ & $1.166(4)$ \\
$\mathrm{N}(2)-\mathrm{N}(3)$ & $1.177(6)$ & $\mathrm{N}(2)-\mathrm{C}(2)$ & $1.148(4)$ \\
$\mathrm{N}(4)-\mathrm{N}(5)$ & $1.170(4)$ & $\mathrm{C}(1)-\mathrm{O}(1)$ & $1.196(4)$ \\
$\mathrm{N}(5)-\mathrm{N}(6)$ & $1.171(4)$ & $\mathrm{C}(2)-\mathrm{O}(2)$ & $1.222(4)$ \\
$\mathrm{B}(1)-\mathrm{N}(6)$ & $1.613(5)$ & $\mathrm{B}(1)-\mathrm{N}(2)$ & $1.563(4)$ \\
$\mathrm{U}(1)-\mathrm{N}(1)-\mathrm{N}(2)$ & $164.2(3)$ & $\mathrm{U}(1)-\mathrm{N}(1)-\mathrm{C}(1)$ & $167.2(3)$ \\
$\mathrm{U}(1)-\mathrm{N}(4)-\mathrm{N}(5)$ & $162.6(2)$ & $\mathrm{U}(1)-\mathrm{O}(2)-\mathrm{C}(2)$ & $167.3(3)$ \\
$\mathrm{N}(5)-\mathrm{N}(6)-\mathrm{B}(1)$ & $132.8(3)$ & $\mathrm{C}(2)-\mathrm{N}(2)-\mathrm{B}(1)$ & $162.1(3)$ \\
$\mathrm{N}(1)-\mathrm{U}(1)-\mathrm{N}(4)$ & $94.0(1)$ & $\mathrm{N}(1)-\mathrm{U}(1)-\mathrm{O}(2)$ & $92.26(9)$ \\
$\mathrm{Cp}($ cent $)-\mathrm{U}$ & $2.466(2), 2.467(2)$ & $\mathrm{Cp}(\mathrm{cent})-\mathrm{U}$ & $2.447(2), 2.449(2)$ \\
$\mathrm{Cp}($ cent $)-\mathrm{U}-\mathrm{Cp}($ cent $)$ & $149.83(5)$ & $\mathrm{Cp}(\mathrm{cent})-\mathrm{U}-\mathrm{Cp}($ cent $)$ & $147.50(5)$
\end{tabular}

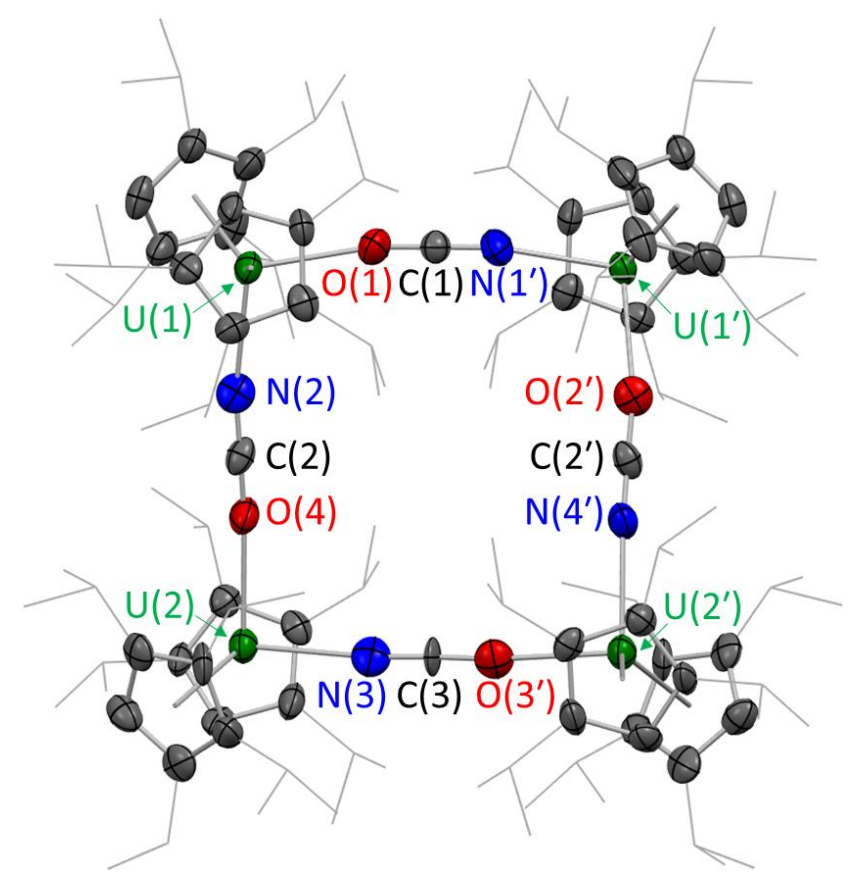

Figure S16. X-ray crystal structure of $\left[\left(\mathrm{Cp}^{\mathrm{iPr} 4}\right)_{2} \mathrm{U}\left(\mu-\eta^{1}: \eta^{1}-\mathrm{NCO}\right)\right]_{4}(3-\mathrm{OCN})$ with $50 \%$ probability ellipsoids. Isopropyl groups are displayed as wireframes; hydrogen atoms and positional disorder are omitted for clarity.

Molecules of 3-OCN lie on a crystallographic 2-fold axis bisecting C(1) and C(3). If the isomer tentatively assigned in Figure S3 is the only isomer present, then molecules of 3-OCN must be disordered in the solid-state such that both $\mathrm{OCN}^{-}$ligand orientations are present in equal ratios 
between each uranium center. In each case, separate positions could not be determined for $\mathrm{O}$ and $\mathrm{N}$ atoms, so in the figure above all $\mathrm{N}$ and $\mathrm{O}$ positions were actually modeled as $50 \% \mathrm{O}+50 \% \mathrm{~N}$ (i.e. $\mathrm{O}(1)=50 \% \mathrm{O}(1)+50 \% \mathrm{~N} 1, \mathrm{~N}(2)=50 \% \mathrm{~N}(2)+50 \% \mathrm{O}(2)$, and so on for $\mathrm{O}(1)-\mathrm{O}(4)$ and $\mathrm{N}(1)-\mathrm{N}(4))$. The crystallographic data therefore does not rule out the presence of any of the isomers in Figure S3, so the isomer displayed in Figure S16 was chosen to be consistent with the assignment discussed in Figure S3. Similar disorder in bridging cyanate/isocyanate ligands was observed in $\left[\mathrm{U}\left\{\eta^{8}-\mathrm{C}_{8} \mathrm{H}_{6}-1,4-\left(\mathrm{Si}^{i} \mathrm{Pr}_{3}\right)_{2}\right\}\left(\mathrm{Cp}^{*}\right)\left(\mu-\eta^{1}: \eta^{1}-\mathrm{OCN}\right)\right]_{2}{ }^{16}$ Selected structural metrics are listed in Table $\mathrm{S} 4$.

Table S4. Selected distances $(\AA)$ and angles $\left(^{\circ}\right)$ for 3-OCN.

\begin{tabular}{ll}
\multicolumn{2}{c}{ 3-OCN } \\
\hline $\mathrm{U}-\mathrm{O} / \mathrm{U}-\mathrm{N}^{\mathrm{a}}$ & $2.476(7)-2.504(7)$ \\
$\mathrm{Cp}($ cent $)-\mathrm{U}^{\mathrm{b}}$ & $2.499(5)-2.542(4)$ \\
$\mathrm{Cp}($ cent $)-\mathrm{U}-\mathrm{Cp}(\text { cent })^{\mathrm{b}}$ & $138.0(2)$
\end{tabular}

${ }^{a}$ As discussed in the caption for Figure S16 above, the orientation of $\mathrm{OCN}^{-}$ligands could not be determined. Since separate positions could not be determined for $\mathrm{O}$ and $\mathrm{N}$ atoms, these distances represent approximate averages between $\mathrm{U}-\mathrm{O}$ and $\mathrm{U}-\mathrm{N}$ bonds.

${ }^{b}$ One of the Cp rings was modeled over two positions; as the AFIX 56 command was used to fix both rings into a regular pentagon (the model was not stable upon refinement when this command was removed), metrics involving these $\mathrm{Cp}$ ring centroids were not included for either component.

Table S5. Selected distances $(\AA)$ and angles $\left(^{\circ}\right)$ for 4-N3 and 4-OCN.

\begin{tabular}{ll|ll}
\multicolumn{2}{c|}{ 4-N3 $_{3}$} & \multicolumn{2}{c}{ 4-OCN } \\
\hline U-F & $2.061(5), 2.068(4)$ & $\mathrm{U}-\mathrm{F}$ & $2.073(2), 2.075(2)$ \\
$\mathrm{U}-\mathrm{N}$ & $2.376(7), 2.381(6)$ & $\mathrm{U}-\mathrm{O}$ & $2.341(2), 2.349(2)$ \\
$\mathrm{N}-\mathrm{N}$ & $1.165(9)-1.191(9)$ & $\mathrm{C}-\mathrm{O}$ & $1.216(5), 1.225(5)$ \\
- & - & $\mathrm{N}-\mathrm{C}$ & $1.150(5), 1.151(5)$ \\
$\mathrm{B}-\mathrm{N}$ & $1.62(1), 1.63(1)$ & $\mathrm{B}-\mathrm{N}$ & $1.551(5), 1.561(5)$ \\
$\mathrm{U}-\mathrm{N}-\mathrm{N}$ & $158.8(6), 161.5(5)$ & $\mathrm{U}-\mathrm{O}-\mathrm{C}$ & $153.8(2), 156.8(2)$ \\
$\mathrm{N}-\mathrm{N}-\mathrm{B}$ & $132.3(6), 136.4(7)$ & $\mathrm{C}-\mathrm{N}-\mathrm{B}$ & $167.6(3), 175.4(3)$ \\
$\mathrm{F}-\mathrm{U}-\mathrm{N}$ & $94.7(2), 95.8(2)$ & $\mathrm{F}-\mathrm{U}-\mathrm{O}$ & $93.79(8), 93.85(8)$ \\
$\mathrm{Cp}($ cent)-U & $2.447(3)-2.459(4)$ & $\mathrm{Cp}($ cent $)-\mathrm{U}$ & $2.443(2)-2.463(2)$ \\
$\mathrm{Cp}($ cent)-U-Cp(cent) & $148.4(1), 152.0(1)$ & $\mathrm{Cp}($ cent)-U-Cp(cent) & $148.35(5), 151.45(5)$
\end{tabular}


Table S6. Crystallographic data for $\left(\mathrm{Cp}^{\mathrm{iPr} 4}\right)_{2} \mathrm{U}\left(\mathrm{N}_{3}\right)_{2}\left(\mathbf{1}-\mathrm{N}_{\mathbf{3}}\right)$

Empirical formula

Formula weight

Color, habit

Temperature

Wavelength

Crystal system

Space group

Unit cell dimensions

Volume

Z

Density (calculated)

Absorption coefficient

$\mathrm{F}(000)$

Crystal size

Theta range for data collection

Index ranges

Reflections collected

Independent reflections

Completeness to theta $=25.242^{\circ}$

Absorption correction

Max. and min. transmission

Refinement method

Data / restraints / parameters

Goodness-of-fit on $\mathrm{F}^{2}$

Final R indices $[\mathrm{I}>2 \sigma(\mathrm{I})]$

$\mathrm{R}$ indices (all data)

Largest diff. peak and hole
$\mathrm{C}_{34} \mathrm{H}_{58} \mathrm{~N}_{6} \mathrm{U}$

788.89

Red, block

100(2) K

$0.71073 \AA$

Monoclinic

$\mathrm{P} 21 / \mathrm{n}$

$\mathrm{a}=10.8145(3) \AA$

$\alpha=90^{\circ}$.

$\mathrm{b}=15.7383(4) \AA$

$\beta=91.8770(10)^{\circ}$.

$\mathrm{c}=21.2659(6) \AA$

$3617.55(17) \AA^{3}$

4

$1.448 \mathrm{Mg} / \mathrm{m}^{3}$

$4.516 \mathrm{~mm}^{-1}$

1584.0

$0.27 \times 0.12 \times 0.11 \mathrm{~mm}^{3}$

1.610 to $25.349^{\circ}$

$-13 \leq \mathrm{h} \leq 13,-18 \leq \mathrm{k} \leq 18,-25 \leq 1 \leq 25$

57992

$6610\left[\mathrm{R}_{\text {int }}=0.0447\right]$

$100.0 \%$

Semi-empirical from equivalents

0.4920 and 0.3275

Full-matrix least-squares on $\mathrm{F}^{2}$

6610 / 0 / 386

1.032

$\mathrm{R}_{1}=0.0187, w \mathrm{R}_{2}=0.0394$

$\mathrm{R}_{1}=0.0267, w \mathrm{R}_{2}=0.0429$

0.64 and $-0.64 \mathrm{e} \cdot \AA^{-3}$ 
Table S7. Crystallographic data for $\left(\mathrm{Cp}^{\mathrm{iPr} 4}\right)_{2} \mathrm{U}(\mathrm{NCO})_{2}(\mathbf{1 - N C O})$

Empirical formula

Formula weight

Color, habit

Temperature

Wavelength

Crystal system

Space group

Unit cell dimensions

Volume

Z

Density (calculated)

Absorption coefficient

$\mathrm{F}(000)$

Crystal size

Theta range for data collection

Index ranges

Reflections collected

Independent reflections

Completeness to theta $=25.242^{\circ}$

Absorption correction

Max. and min. transmission

Refinement method

Data / restraints / parameters

Goodness-of-fit on $\mathrm{F}^{2}$

Final R indices $[\mathrm{I}>2 \sigma(\mathrm{I})]$

$\mathrm{R}$ indices (all data)

Largest diff. peak and hole
$\mathrm{C}_{36} \mathrm{H}_{58} \mathrm{~N}_{2} \mathrm{O}_{2} \mathrm{U}$

788.87

Red, block

100(2) K

$0.71073 \AA$

Monoclinic

$\mathrm{P} 21 / \mathrm{n}$

$\mathrm{a}=10.7657(3) \AA \quad \alpha=90^{\circ}$.

$\mathrm{b}=15.6950(6) \AA$

$\beta=91.912(3)^{\circ}$.

$c=21.3659(7) \AA$

3608.1(2) $\AA^{3}$

$\gamma=90^{\circ}$.
4

$1.452 \mathrm{Mg} / \mathrm{m}^{3}$

$4.529 \mathrm{~mm}^{-1}$

1584.0

$0.24 \times 0.16 \times 0.12 \mathrm{~mm}^{3}$

2.944 to $25.349^{\circ}$

$-12 \leq \mathrm{h} \leq 12,-18 \leq \mathrm{k} \leq 18,-25 \leq 1 \leq 25$

71284

$6581\left[\mathrm{R}_{\mathrm{int}}=0.0976\right]$

$99.8 \%$

Semi-empirical from equivalents

1.00000 and 0.39374

Full-matrix least-squares on $\mathrm{F}^{2}$

$6581 / 0 / 386$

1.029

$\mathrm{R}_{1}=0.0269, w \mathrm{R}_{2}=0.0683$

$\mathrm{R}_{1}=0.0347, w \mathrm{R}_{2}=0.0726$

1.18 and $-1.36 \mathrm{e} \cdot \AA^{-3}$ 
Table S8. Crystallographic data for $\left(\mathrm{Cp}^{\mathrm{iPr} 4}\right)_{2} \mathrm{U}(\mathrm{OTf})_{2}$ (1-OTf)

Empirical formula

Formula weight

Color, habit

Temperature

Wavelength

Crystal system

Space group

Unit cell dimensions

Volume

Z

Density (calculated)

Absorption coefficient

$\mathrm{F}(000)$

Crystal size

Theta range for data collection

Index ranges

Reflections collected

Independent reflections

Completeness to theta $=25.930^{\circ}$

Absorption correction

Max. and min. transmission

Refinement method

Data / restraints / parameters

Goodness-of-fit on $\mathrm{F}^{2}$

Final $\mathrm{R}$ indices $[\mathrm{I}>2 \sigma(\mathrm{I})]$

$\mathrm{R}$ indices (all data)

Largest diff. peak and hole
$\mathrm{C}_{43} \mathrm{H}_{66} \mathrm{~F}_{6} \mathrm{O}_{6} \mathrm{~S}_{2} \mathrm{U}$

1095.11

Orange, plate

100(2) K

$0.7288 \AA$

Monoclinic

$\mathrm{C} 2 / \mathrm{c}$

$\mathrm{a}=40.856(2) \AA$

$\alpha=90^{\circ}$.

$\mathrm{b}=12.5871(8) \AA$

$\beta=117.187(2)^{\circ}$.

$\mathrm{c}=18.9238(12) \AA$

$\gamma=90^{\circ}$.
8

$1.680 \mathrm{Mg} / \mathrm{m}^{3}$

$1.893 \mathrm{~mm}^{-1}$

4000

$0.050 \times 0.050 \times 0.005 \mathrm{~mm}^{3}$

1.756 to $26.133^{\circ}$

$-47 \leq \mathrm{h} \leq 47,-15 \leq \mathrm{k} \leq 15,-22 \leq 1 \leq 22$

27231

$7643\left[\mathrm{R}_{\text {int }}=0.1061\right]$

$95.8 \%$

Semi-empirical from equivalents

0.7453 and 0.4869

Full-matrix least-squares on $\mathrm{F}^{2}$

7643 / 0 / 476

1.017

$\mathrm{R}_{1}=0.0465, w \mathrm{R}_{2}=0.1055$

$\mathrm{R}_{1}=0.0763, w \mathrm{R}_{2}=0.1175$

2.058 and $-1.791 \mathrm{e} \cdot \AA^{-3}$ 
Table S9. Crystallographic data for $\left(\mathrm{Cp}^{\mathrm{iPr} 4}\right)_{2} \mathrm{U}\left(\mathrm{N}_{3}\right)(\mathrm{OTf})\left(\mathbf{1}-\mathrm{N}_{3}-\mathrm{OTf}\right)$

Empirical formula

Formula weight

Color, habit

Temperature

Wavelength

Crystal system

Space group

Unit cell dimensions

Volume

Z

Density (calculated)

Absorption coefficient

$\mathrm{F}(000)$

Crystal size

Theta range for data collection

Index ranges

Reflections collected

Independent reflections

Completeness to theta $=25.242^{\circ}$

Absorption correction

Max. and min. transmission

Refinement method

Data / restraints / parameters

Goodness-of-fit on $\mathrm{F}^{2}$

Final R indices $[\mathrm{I}>2 \sigma(\mathrm{I})]$

$\mathrm{R}$ indices (all data)

Largest diff. peak and hole
$\mathrm{C}_{35} \mathrm{H}_{58} \mathrm{~F}_{3} \mathrm{~N}_{3} \mathrm{O}_{3} \mathrm{SU}$

895.93

Orange, plate

100(2) K

$0.71073 \AA$

Monoclinic

$\mathrm{P} 21 / \mathrm{n}$

$\mathrm{a}=9.6930(4) \AA \quad \alpha=90^{\circ}$.

$\mathrm{b}=20.3432(9) \AA \quad \beta=90.421(2)^{\circ}$.

$\mathrm{c}=19.1575(8) \AA \quad \gamma=90^{\circ}$.

$3777.5(3) \AA^{3}$

4

$1.575 \mathrm{Mg} / \mathrm{m}^{3}$

$4.403 \mathrm{~mm}^{-1}$

1792.0

$0.030 \times 0.012 \times 0.010 \mathrm{~mm}^{3}$

1.460 to $25.365^{\circ}$

$-11 \leq \mathrm{h} \leq 11,-23 \leq \mathrm{k} \leq 24,-23 \leq 1 \leq 21$

36068

$6904\left[\mathrm{R}_{\text {int }}=0.0751\right]$

$99.7 \%$

Semi-empirical from equivalents

0.7452 and 0.6047

Full-matrix least-squares on $\mathrm{F}^{2}$

6904 / 6 / 431

1.028

$\mathrm{R}_{1}=0.0407, w \mathrm{R}_{2}=0.0841$

$\mathrm{R}_{1}=0.0696, w \mathrm{R}_{2}=0.0941$

2.94 and $-1.38 \mathrm{e} \cdot \AA^{-3}$ 
Table S10. Crystallographic data for $\left(\mathrm{Cp}^{\mathrm{iPr} 4}\right)_{2} \mathrm{U}\left(\mathrm{N}_{3}\right)\left[\left(\mu-\eta^{1}: \eta^{1}-\mathrm{N}_{3}\right) \mathrm{B}\left(\mathrm{C}_{6} \mathrm{~F}_{5}\right)_{3}\right]\left(\mathbf{2}-\mathrm{N}_{3}\right)$

Empirical formula

Formula weight

Color, habit

Temperature

Wavelength

Crystal system

Space group

Unit cell dimensions

Volume

Z

Density (calculated)

Absorption coefficient

$\mathrm{F}(000)$

Crystal size

Theta range for data collection

Index ranges

Reflections collected

Independent reflections

Completeness to theta $=25.930^{\circ}$

Absorption correction

Max. and min. transmission

Refinement method

Data / restraints / parameters

Goodness-of-fit on $\mathrm{F}^{2}$

Final R indices $[\mathrm{I}>2 \sigma(\mathrm{I})]$

$\mathrm{R}$ indices (all data)

Largest diff. peak and hole
$\mathrm{C}_{52} \mathrm{H}_{58} \mathrm{BF}_{15} \mathrm{~N}_{6} \mathrm{U}$

1300.88

Red, needle

100.0(2) K

$0.7288 \AA$

Monoclinic

$\mathrm{P} 21 / \mathrm{n}$

$\mathrm{a}=14.2921(6) \AA \quad \alpha=90^{\circ}$.

$\mathrm{b}=22.1342(9) \AA \quad \beta=93.049(2)^{\circ}$.

$\mathrm{c}=16.7847(7) \AA \quad \gamma=90^{\circ}$.

$5302.2(4) \AA^{3}$

4

$1.630 \mathrm{Mg} / \mathrm{m}^{3}$

$1.506 \mathrm{~mm}^{-1}$

2576.0

$0.130 \times 0.020 \times 0.010 \mathrm{~mm}^{3}$

1.972 to $26.042^{\circ}$

$-17 \leq \mathrm{h} \leq 17,-26 \leq \mathrm{k} \leq 26,-20 \leq 1 \leq 20$

72723

$9701\left[\mathrm{R}_{\mathrm{int}}=0.0677\right]$

$99.9 \%$

Semi-empirical from equivalents

0.7458 and 0.6297

Full-matrix least-squares on $\mathrm{F}^{2}$

$9701 / 1 / 692$

1.028

$\mathrm{R}_{1}=0.0307, w \mathrm{R}_{2}=0.0727$

$\mathrm{R}_{1}=0.0370, w \mathrm{R}_{2}=0.0769$

1.05 and $-0.91 \mathrm{e} \cdot \AA^{-3}$ 
Table S11. Crystallographic data for $\left(\mathrm{Cp}^{\mathrm{iPr} 4}\right)_{2} \mathrm{U}(\mathrm{NCO})\left[\left(\mu-\eta^{1}: \eta^{1}-\mathrm{OCN}\right) \mathrm{B}\left(\mathrm{C}_{6} \mathrm{~F}_{5}\right)_{3}\right](\mathbf{2}-\mathrm{NCO})$

Empirical formula

Formula weight

Color, habit

Temperature

Wavelength

Crystal system

Space group

Unit cell dimensions

Volume

Z

Density (calculated)

Absorption coefficient

$\mathrm{F}(000)$

Crystal size

Theta range for data collection

Index ranges

Reflections collected

Independent reflections

Completeness to theta $=25.930^{\circ}$

Absorption correction

Max. and min. transmission

Refinement method

Data / restraints / parameters

Goodness-of-fit on $\mathrm{F}^{2}$

Final R indices $[\mathrm{I}>2 \sigma(\mathrm{I})]$

$\mathrm{R}$ indices (all data)

Largest diff. peak and hole
$\mathrm{C}_{54} \mathrm{H}_{58} \mathrm{BF}_{15} \mathrm{~N}_{2} \mathrm{O}_{2} \mathrm{U}$

1300.86

Orange, plate

100(2) K

$0.7288 \AA$

Monoclinic

$\mathrm{P} 21 / \mathrm{n}$

$\mathrm{a}=12.8889(4) \AA \quad \alpha=90^{\circ}$.

$\mathrm{b}=26.8415(9) \AA \quad \beta=110.2300(10)^{\circ}$.

$\mathrm{c}=15.9139(6) \AA \quad \gamma=90^{\circ}$.

5165.9(3) $\AA^{3}$

4

$1.673 \mathrm{Mg} / \mathrm{m}^{3}$

$1.546 \mathrm{~mm}^{-1}$

2576.0

$0.025 \times 0.025 \times 0.010 \mathrm{~mm}^{3}$

1.556 to $26.042^{\circ}$

$-15 \leq \mathrm{h} \leq 15,-32 \leq \mathrm{k} \leq 32,-19 \leq 1 \leq 19$

68734

$9455\left[\mathrm{R}_{\text {int }}=0.0598\right]$

$99.9 \%$

Semi-empirical from equivalents

0.7462 and 0.6711

Full-matrix least-squares on $\mathrm{F}^{2}$

9455 / 0 / 692

1.055

$\mathrm{R}_{1}=0.0276, w \mathrm{R}_{2}=0.0624$

$\mathrm{R}_{1}=0.0317, w \mathrm{R}_{2}=0.0649$

0.88 and $-0.63 \mathrm{e} \cdot \AA^{-3}$ 
Table S12. Crystallographic data for $\left[\left(\mathrm{Cp}^{\mathrm{iPr} 4}\right)_{2} \mathrm{U}\left(\mu-\eta^{1}: \eta^{1}-\mathrm{NCO}\right)\right]_{4}(3-\mathrm{OCN})$

Empirical formula

Formula weight

Color, habit

Temperature

Wavelength

Crystal system

Space group

Unit cell dimensions

Volume

$\mathrm{Z} / \mathrm{Z}^{\prime \prime}$

Density (calculated)

Absorption coefficient

$\mathrm{F}(000)$

Crystal size

Theta range for data collection

Index ranges

Reflections collected

Independent reflections

Completeness to theta $=25.930^{\circ}$

Absorption correction

Max. and min. transmission

Refinement method

Data / restraints / parameters

Goodness-of-fit on $\mathrm{F}^{2}$

Final R indices $[\mathrm{I}>2 \sigma(\mathrm{I})]$

$\mathrm{R}$ indices (all data)

Flack parameter

Largest diff. peak and hole
$\mathrm{C}_{140} \mathrm{H}_{232} \mathrm{~N}_{4} \mathrm{O}_{4} \mathrm{U}_{4}$

2987.40

Green, cube

100(2) K

$0.7288 \AA$

Tetragonal

$\mathrm{P}_{2} 2{ }_{1} 2$

$\mathrm{a}=20.8792(7) \AA \quad \alpha=90^{\circ}$.

$\mathrm{b}=20.8792(7) \AA \quad \beta=90^{\circ}$.

$\mathrm{c}=35.3748(13) \AA \quad \gamma=90^{\circ}$.

15421.3(12) $\AA^{3}$

$8 / 4$

$1.287 \mathrm{Mg} / \mathrm{m}^{3}$

$1.957 \mathrm{~mm}^{-1}$

6000.0

$0.030 \times 0.030 \times 0.030 \mathrm{~mm}^{3}$

2.000 to $26.024^{\circ}$

$-25 \leq \mathrm{h} \leq 25,-25 \leq \mathrm{k} \leq 24,-42 \leq 1 \leq 42$

209076

$14107\left[\mathrm{R}_{\text {int }}=0.0901\right]$

$99.9 \%$

Semi-empirical from equivalents

0.7457 and 0.5723

Full-matrix least-squares on $\mathrm{F}^{2}$

14107 / 61 / 815

1.120

$\mathrm{R}_{1}=0.0397, w \mathbf{R}_{2}=0.0929$

$\mathrm{R}_{1}=0.0414, w \mathrm{R}_{2}=0.0936$

$0.62(2)$

1.01 and $-1.02 \mathrm{e} \cdot \AA^{-3}$ 
Table S13. Crystallographic data for $\left(\mathrm{Cp}^{\mathrm{iPr} 4}\right)_{2} \mathrm{U}(\mathrm{F})\left(\mu-\eta^{1}: \eta^{1}-\mathrm{OCN}\right) \mathrm{B}\left(\mathrm{C}_{6} \mathrm{~F}_{5}\right)_{3}(\mathbf{4 - O C N})$

Empirical formula

Formula weight

Color, habit

Temperature

Wavelength

Crystal system

Space group

Unit cell dimensions

Volume

Z / Z"

Density (calculated)

Absorption coefficient

$\mathrm{F}(000)$

Crystal size

Theta range for data collection

Index ranges

Reflections collected

Independent reflections

Completeness to theta $=25.930^{\circ}$

Absorption correction

Max. and min. transmission

Refinement method

Data / restraints / parameters

Goodness-of-fit on $\mathrm{F}^{2}$

Final R indices $[\mathrm{I}>2 \sigma(\mathrm{I})]$

$\mathrm{R}$ indices (all data)

Largest diff. peak and hole
$\mathrm{C}_{53} \mathrm{H}_{58} \mathrm{BF}_{16} \mathrm{NOU}$

1277.84

Orange, plate

100(2) K

$0.7288 \AA$

Triclinic

$\mathrm{P}-1$

$\mathrm{a}=14.1777(5) \AA \quad \alpha=84.9080(10)^{\circ}$.

$\mathrm{b}=16.6086(6) \AA \quad \beta=77.990(2)^{\circ}$.

$\mathrm{c}=22.5965(8) \AA \quad \gamma=83.2760(10)^{\circ}$.

$5157.2(3) \AA^{3}$

$2 / 4$

$1.646 \mathrm{Mg} / \mathrm{m}^{3}$

$1.548 \mathrm{~mm}^{-1}$

2528.0

$0.080 \times 0.080 \times 0.030 \mathrm{~mm}^{3}$

1.269 to $26.042^{\circ}$

$-17 \leq \mathrm{h} \leq 17,-20 \leq \mathrm{k} \leq 20,-27 \leq 1 \leq 27$

69230

$18869\left[\mathrm{R}_{\text {int }}=0.0426\right]$

$100.0 \%$

Semi-empirical from equivalents

0.7462 and 0.6285

Full-matrix least-squares on $\mathrm{F}^{2}$

18869 / 0 / 1347

1.045

$\mathrm{R}_{1}=0.0291, w \mathrm{R}_{2}=0.0670$

$\mathrm{R}_{1}=0.0357, w \mathrm{R}_{2}=0.0702$

2.88 and $-0.89 \mathrm{e} \cdot \AA^{-3}$ 
Table S14. Crystallographic data for $\left(\mathrm{Cp}^{\mathrm{iPr} 4}\right)_{2} \mathrm{U}(\mathrm{F})\left(\mu-\eta^{1}: \eta^{1}-\mathrm{N}_{3}\right) \mathrm{B}\left(\mathrm{C}_{6} \mathrm{~F}_{5}\right)_{3}\left(\mathbf{4}-\mathrm{N}_{3}\right)$

Empirical formula

Formula weight

Color, habit

Temperature

Wavelength

Crystal system

Space group

Unit cell dimensions

Volume

Z / Z"

Density (calculated)

Absorption coefficient

$\mathrm{F}(000)$

Crystal size

Theta range for data collection

Index ranges

Reflections collected

Independent reflections

Completeness to theta $=25.930^{\circ}$

Absorption correction

Max. and min. transmission

Refinement method

Data / restraints / parameters

Goodness-of-fit on $\mathrm{F}^{2}$

Final R indices $[\mathrm{I}>2 \sigma(\mathrm{I})]$

$\mathrm{R}$ indices (all data)

Largest diff. peak and hole
$\mathrm{C}_{52} \mathrm{H}_{58} \mathrm{BF}_{16} \mathrm{~N}_{3} \mathrm{U}$

1277.85

Orange, needle

100(2) K

$0.7288 \AA$

Monoclinic

$\mathrm{P} 21 / \mathrm{c}$

$\mathrm{a}=23.1009(13) \AA \quad \alpha=90^{\circ}$.

$\mathrm{b}=16.8277(10) \AA \quad \beta=110.023(2)^{\circ}$.

$\mathrm{c}=28.5122(16) \AA \quad \gamma=90^{\circ}$.

10413.7(10) $\AA^{3}$

$4 / 8$

$1.630 \mathrm{Mg} / \mathrm{m}^{3}$

$1.533 \mathrm{~mm}^{-1}$

5056.0

$0.080 \times 0.015 \times 0.015 \mathrm{~mm}^{3}$

1.526 to $26.042^{\circ}$

$-27 \leq \mathrm{h} \leq 27,-20 \leq \mathrm{k} \leq 20,-34 \leq 1 \leq 34$

137706

$19065\left[R_{\text {int }}=0.0790\right]$

$99.9 \%$

Semi-empirical from equivalents

0.7457 and 0.5675

Full-matrix least-squares on $\mathrm{F}^{2}$

19065 / 0 / 1347

1.090

$\mathrm{R}_{1}=0.0603, w \mathrm{R}_{2}=0.1381$

$\mathrm{R}_{1}=0.0716, w \mathrm{R}_{2}=0.1432$

3.22 and $-3.38 \mathrm{e} \cdot \AA^{-3}$ 


\section{Electrochemistry}

Cyclic voltammetry (CV) experiments were performed with a Gamry Reference 600 ${ }^{\mathrm{TM}}$ potentiostat using screen-printed electrodes (SPEs) manufactured by Pine Research Instrumentation. The SPEs have platinum working and counter electrodes and a built-in silver pseudo-reference electrode. The electrodes are screen-printed onto a ceramic substrate such that the three electrodes are in close proximity to each other, without touching, at all times. The measurements were conducted in a dry N2 atmosphere glovebox with 2-3 mM analyte in $0.1 \mathrm{M}$ solutions of $\left[{ }^{n} \mathrm{Bu}_{4} \mathrm{~N}\right]\left[\mathrm{B}\left(\mathrm{C}_{6} \mathrm{~F}_{5}\right)_{4}\right]$ in THF at room temperature. Potentials were referenced versus the ferrocene $(\mathrm{Fc}) /$ ferrocenium $\left(\mathrm{Fc}^{+}\right)$redox couple by adding $\mathrm{Fc}$ (sublimed) as an internal standard for calibration at the end of each set of measurements. 


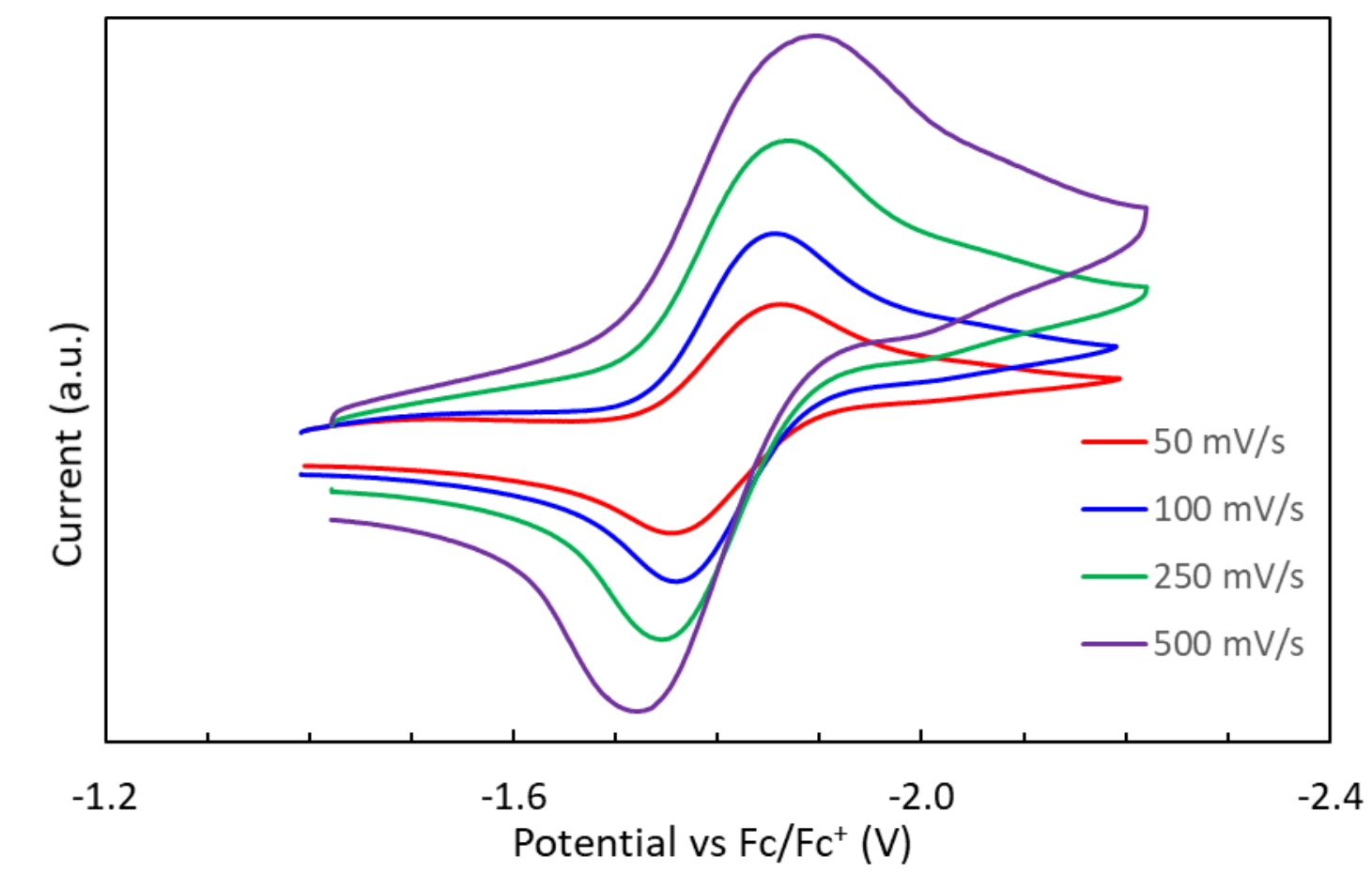

Figure S17. Scan rate dependent measurements of the uranium(III/IV) redox couple for 1-N3.

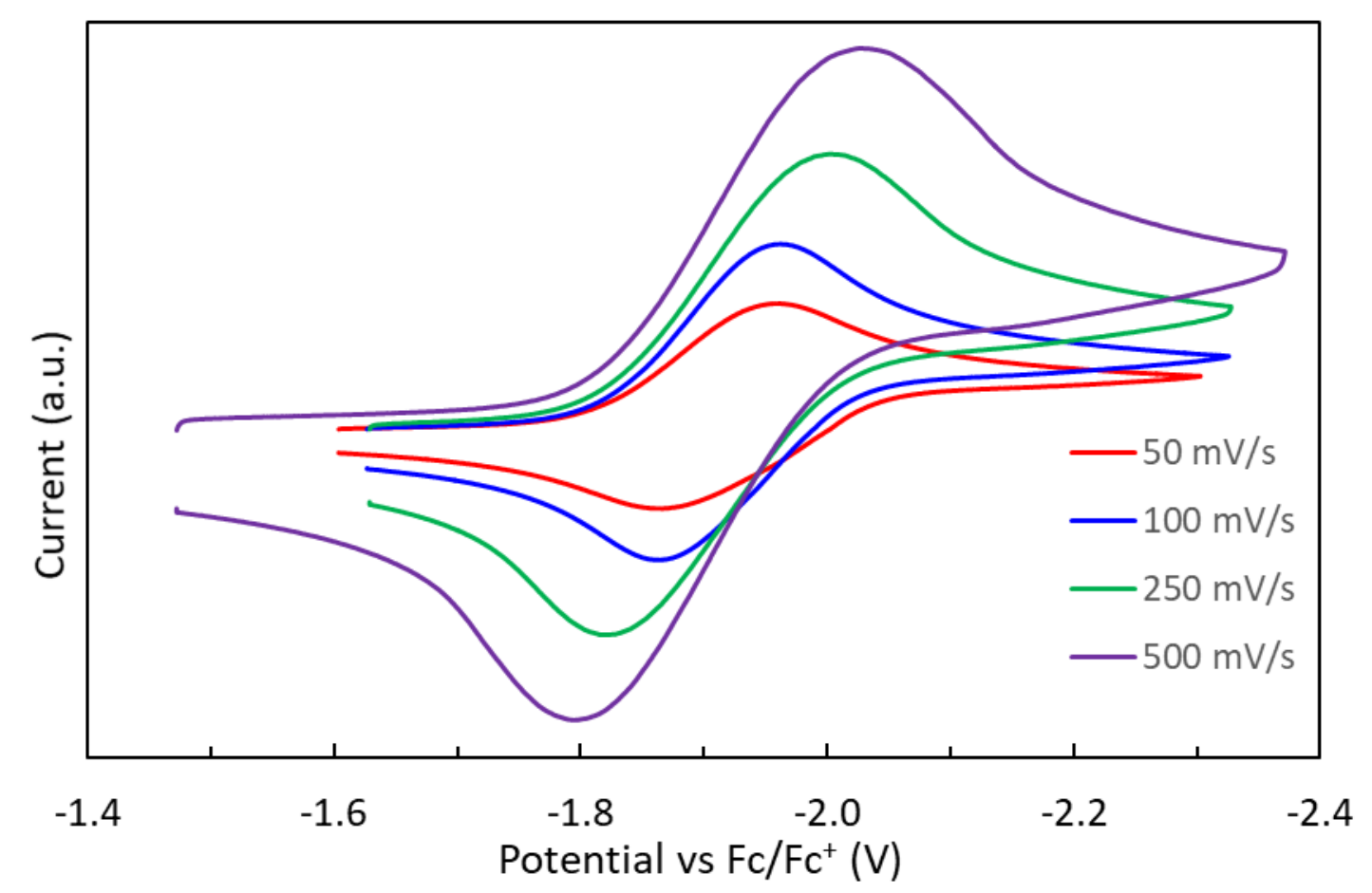

Figure S18. Scan rate dependent measurements of the uranium(II//IV) redox couple for 1-NCO. 


\section{UV-Visible Spectroscopy}

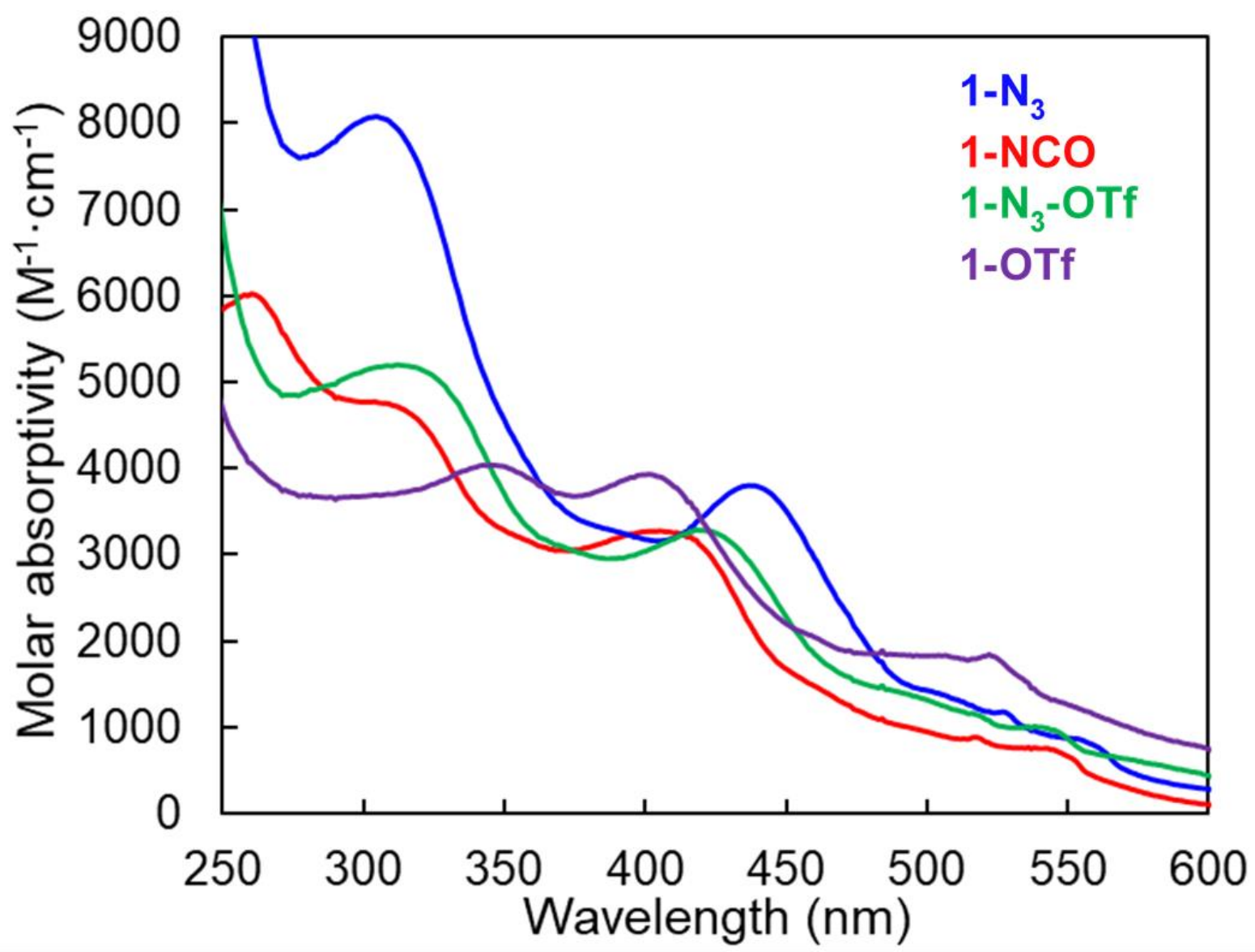

Figure S19. UV-visible spectra of 1-N3, 1-NCO, 1-N3-OTf, 1-OTf in THF.

Table S15. Experimental energies and intensities of the charge transfer bands of 1-N3, 1-NCO, 1N3-OTf, 1-OTf in THF solutions

\begin{tabular}{ccc} 
Complex & $\lambda_{\max }(\mathrm{nm}), v_{\max }\left(\mathrm{cm}^{-1}\right)$ & $\varepsilon_{\max }\left(\mathrm{M}^{-1} \cdot \mathrm{cm}^{-1}\right)$ \\
\hline 1-N3 & 437,22900 & 3800 \\
& 305,32800 & 8080 \\
1-NCO & 405,24700 & 3280 \\
& 309,32400 & 4750 \\
& 260,38500 & 6020 \\
1-N3-OTf & 418,23900 & 3290 \\
& 312,32000 & 5200 \\
1-OTf & 401,24900 & 3930 \\
& 345,29000 & 4040
\end{tabular}




\section{Computational Section}

All the structures reported in this study were fully optimized with the Becke's 3-parameter hybrid functional ${ }^{17}$ combined with the non-local correlation functional provided by Perdew/Wang (denoted as B3PW91). ${ }^{18}$ The basis set used for uranium atom was the Stuttgart-Dresden small core ECP in combination with its adapted basis set. ${ }^{19,20}$ For the rest of the atoms the 6-31G(d,p) basis set was used. ${ }^{21-23}$ In all computations no constraints were imposed on the geometry. All stationary points have been identified for minimum (number of imaginary frequencies $\mathrm{N}_{\mathrm{imag}}=0$ ) or transition states $\left(\mathrm{N}_{\mathrm{imag}}=1\right)$. The vibrational modes and the corresponding frequencies are based on a harmonic force field. Enthalpy energies were obtained at $\mathrm{T}=298.15 \mathrm{~K}$ within the harmonic approximation. GAUSSIAN09 program suite was used in all calculations. ${ }^{24}$ Density was analyzed using NBO methods. ${ }^{25}$ Alpha and beta orbitals were identical in the description of the density about the U-ligand system in all cases.

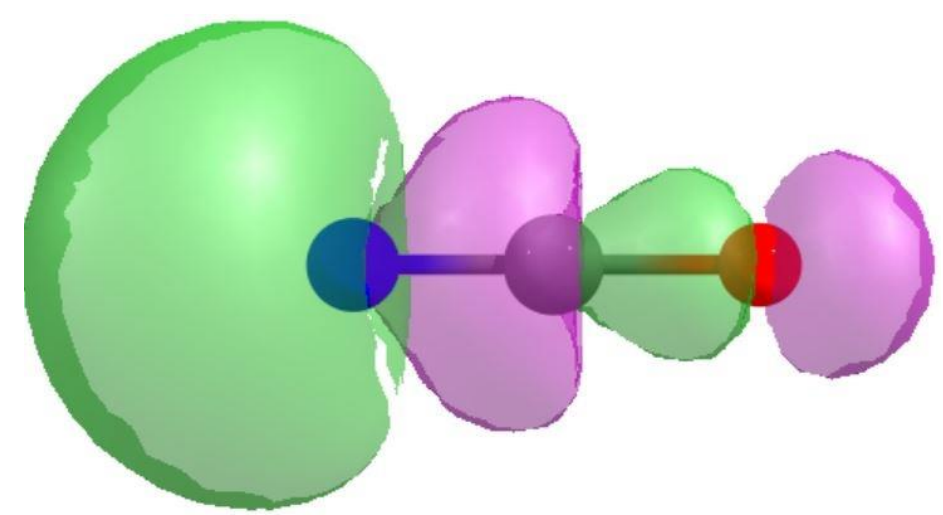

Figure S20. HOMO of the free $\mathrm{NCO}^{-}$ion. 


\section{1-NCO}

Isomer 1-N,N (Energy $=-2142.49$ Hartrees)

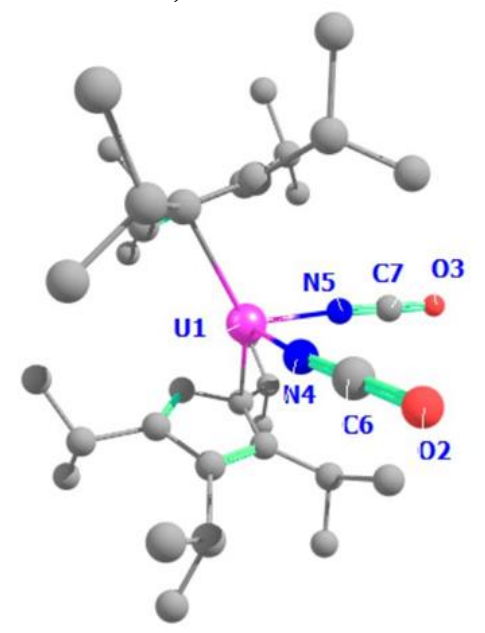

Figure S21. Optimized structure of 1-N,N (uranium: pink, carbon: gray, nitrogen: blue, oxygen: red).

Table S16. Selected calculated metrics for $\mathbf{1 - N}, \mathbf{N}$.

\begin{tabular}{|c|c|c|c|c|}
\hline Bond Coordinate & Distance $(\AA)$ & WBI & Bond Coordinate & Angle $\left(^{\circ}\right)$ \\
\hline U1-N5 & 2.23 & 0.8 & N4-U1-N5 & 94.3 \\
\hline N5-C7 & 1.21 & 1.5 & U1-N5-C7 & 171.9 \\
\hline $\mathrm{C} 7-\mathrm{O} 3$ & 1.18 & 1.4 & U1-N4-C6 & 172.0 \\
\hline $\mathrm{U} 1-\mathrm{N} 4$ & 2.23 & 0.8 & N5-C7-O3 & 179.6 \\
\hline N4-C6 & 1.21 & 1.5 & $\mathrm{O} 2-\mathrm{C} 6-\mathrm{N} 4$ & 179.6 \\
\hline $\mathrm{C} 6-\mathrm{O} 2$ & 1.18 & 1.4 & & \\
\hline
\end{tabular}


Table S17. Relevant natural bonding orbitals in 1-N,N.

\begin{tabular}{|cccc|cccc|}
\multicolumn{4}{c}{ Or } & \multicolumn{4}{c|}{ C6 } \\
\hline \multicolumn{4}{c}{$65.17 \%$} & & \multicolumn{4}{c|}{${ }^{34.83 \%}$} & $\mathrm{f}$ \\
$\mathrm{s}$ & $\mathrm{p}$ & $\mathrm{d}$ & $\mathrm{f}$ & $\mathrm{s}$ & $\mathrm{p}$ & $\mathrm{d}$ & $\mathrm{f}$ \\
$39.84 \%$ & $59.81 \%$ & $0.00 \%$ & $0.00 \%$ & $46.10 \%$ & $53.80 \%$ & $0.00 \%$ & $0.00 \%$ \\
\hline
\end{tabular}

\begin{tabular}{|cccc|cccc|}
\multicolumn{4}{c}{$\mathbf{0 3}$} & \multicolumn{4}{c|}{$\mathbf{C 7}$} \\
\hline \multicolumn{4}{|c}{$65.17 \%$} & & \multicolumn{4}{c|}{$34.83 \%$} & $\mathrm{~d}$ & $\mathrm{f}$ \\
$\mathrm{s}$ & $\mathrm{p}$ & $\mathrm{d}$ & $\mathrm{f}$ & $\mathrm{s}$ & $\mathrm{p}$ & $\mathrm{d}$ & $0.00 \%$ \\
$39.84 \%$ & $59.81 \%$ & $0.00 \%$ & $0.00 \%$ & $46.10 \%$ & $53.80 \%$ & $0.00 \%$ & $0.00 \%$ \\
\hline
\end{tabular}

\begin{tabular}{|cccc|cccc|}
\multicolumn{4}{c}{ Nu } & \multicolumn{5}{c|}{ CG } \\
\hline \multicolumn{4}{c}{$60.27 \%$} & & \multicolumn{4}{c|}{$39.73 \%$} & $\mathrm{~d}$ & $\mathrm{f}$ \\
$\mathrm{s}$ & $\mathrm{p}$ & $\mathrm{d}$ & $\mathrm{f}$ & $\mathrm{s}$ & $\mathrm{p}$ & $\mathrm{d}$ & \\
$54.79 \%$ & $45.17 \%$ & $0.00 \%$ & $0.00 \%$ & $53.66 \%$ & $46.28 \%$ & $0.00 \%$ & $0.00 \%$ \\
\hline
\end{tabular}

\begin{tabular}{|cccc|cccc|}
\hline \multicolumn{4}{|c|}{$70.58 \%$} & \multicolumn{4}{c|}{$29.42 \%$} \\
$\mathrm{~s}$ & $\mathrm{p}$ & $\mathrm{d}$ & $\mathrm{f}$ & $\mathrm{s}$ & $\mathrm{p}$ & $\mathrm{d}$ & $\mathrm{f}$ \\
$0.03 \%$ & $99.97 \%$ & $0.00 \%$ & $0.00 \%$ & $0.01 \%$ & $99.99 \%$ & $0.00 \%$ & $0.00 \%$ \\
\hline
\end{tabular}

\begin{tabular}{|cccc|cccc|}
\hline \multicolumn{4}{|c|}{$71.07 \%$} & \multicolumn{4}{c|}{$28.93 \%$} \\
$\mathrm{~s}$ & $\mathrm{p}$ & $\mathrm{d}$ & $\mathrm{f}$ & $\mathrm{s}$ & $\mathrm{p}$ & $\mathrm{d}$ & $\mathrm{f}$ \\
$0.01 \%$ & $99.91 \%$ & $0.08 \%$ & $0.00 \%$ & $0.06 \%$ & $99.67 \%$ & $0.27 \%$ & $0.00 \%$ \\
\hline
\end{tabular}

\begin{tabular}{|cccc|cccc|}
\multicolumn{4}{c}{ N5 } & \multicolumn{4}{c|}{ C7 } \\
\hline \multicolumn{4}{c}{$60.27 \%$} & & \multicolumn{4}{c|}{$39.73 \%$} & $\mathrm{~d}$ & $\mathrm{f}$ \\
$\mathrm{s}$ & $\mathrm{p}$ & $\mathrm{d}$ & $\mathrm{f}$ & $\mathrm{s}$ & $\mathrm{p}$ & $\mathrm{d}$ & \\
$54.79 \%$ & $45.17 \%$ & $0.00 \%$ & $0.00 \%$ & $53.66 \%$ & $46.28 \%$ & $0.00 \%$ & $0.00 \%$ \\
\hline
\end{tabular}

\begin{tabular}{|cccc|cccc|}
\hline \multicolumn{4}{|c|}{$70.58 \%$} & \multicolumn{4}{c|}{$29.42 \%$} \\
$\mathrm{~s}$ & $\mathrm{p}$ & $\mathrm{d}$ & $\mathrm{f}$ & $\mathrm{s}$ & $\mathrm{p}$ & $\mathrm{d}$ & $\mathrm{f}$ \\
$0.03 \%$ & $99.97 \%$ & $0.00 \%$ & $0.00 \%$ & $0.01 \%$ & $99.99 \%$ & $0.00 \%$ & $0.00 \%$ \\
\hline
\end{tabular}

\begin{tabular}{|cccc|cccc|}
\hline \multicolumn{4}{|c|}{$71.07 \%$} & \multicolumn{4}{c|}{$28.93 \%$} \\
$\mathrm{~s}$ & $\mathrm{p}$ & $\mathrm{d}$ & $\mathrm{f}$ & $\mathrm{s}$ & $\mathrm{p}$ & $\mathrm{d}$ & $\mathrm{f}$ \\
$0.01 \%$ & $99.91 \%$ & $0.08 \%$ & $0.00 \%$ & $0.06 \%$ & $99.67 \%$ & $0.27 \%$ & $0.00 \%$ \\
\hline
\end{tabular}

S40 
Isomer 1-O,0 (Energy $=-2142.43$ Hartrees $)$

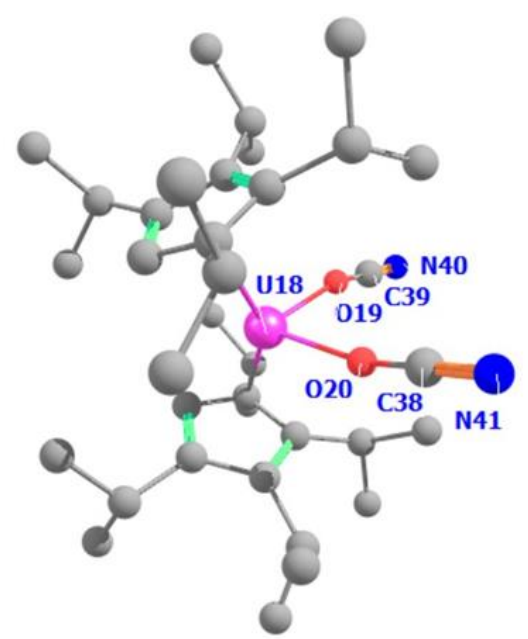

Figure S22. Optimized structure of 1-O,O (uranium: pink, carbon: gray, nitrogen: blue, oxygen: red).

Table S18. Selected calculated metrics for 1-0,0.

\begin{tabular}{lll|ll} 
Bond Coordinate & Distance $(\AA)$ & WBI & Bond Coordinate & Angle $\left(^{\circ}\right)$ \\
U18-O19 & 2.19 & 0.6 & O20-U18-O19 & 92.9 \\
U18-O20 & 2.19 & 0.6 & U18-O19-C39 & 164.4 \\
O19-C39 & 1.26 & 1.2 & U18-O20-C38 & 164.3 \\
O20-C38 & 1.26 & 1.2 & O19-C39-N40 & 179.3 \\
C39-N40 & 1.17 & 2.7 & O20-C38-N41 & 179.4 \\
C38-N41 & 1.17 & 2.7 & &
\end{tabular}
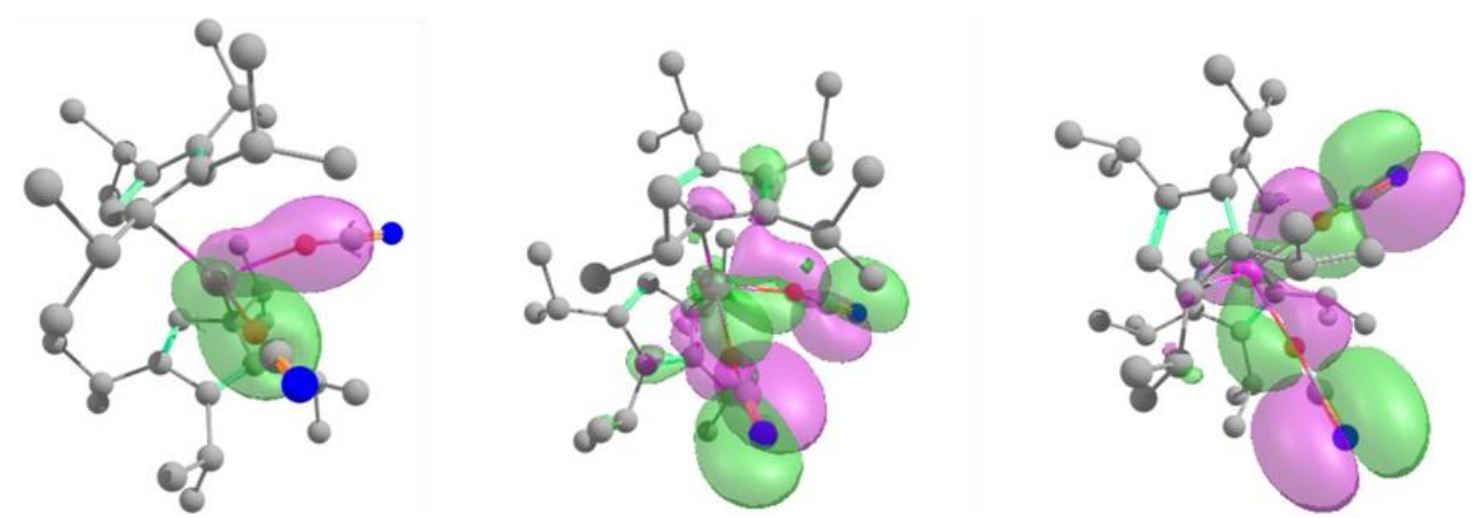

Figure S23. MOs depicting the $\sigma$ interactions between the $\mathrm{O}$ and $\mathrm{U}$ atoms (left), and the $p$ orbital interactions with the $d_{x y}$ (middle) and $d_{x 2-y 2}$ (right) of the $U$ atom in 1-O,O (uranium: pink, carbon: gray, nitrogen: blue, oxygen: red). 
Table S19. Relevant natural bonding orbitals in 1-O,O.

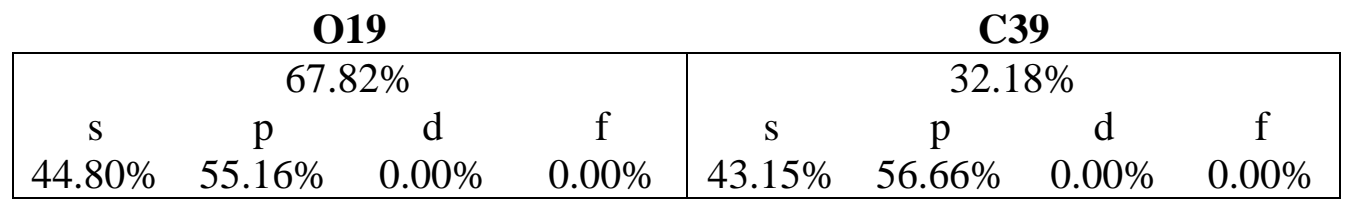

\begin{tabular}{|cccc|cccc|}
\multicolumn{4}{c}{ O20 } & \multicolumn{4}{c|}{ C38 } \\
\hline \multicolumn{4}{c}{$67.82 \%$} & & \multicolumn{4}{c|}{$32.18 \%$} & $\mathrm{~d}$ & $\mathrm{f}$ \\
$\mathrm{s}$ & $\mathrm{p}$ & $\mathrm{d}$ & $\mathrm{f}$ & $\mathrm{s}$ & $\mathrm{p}$ & $\mathrm{d}$ & \\
$44.80 \%$ & $55.16 \%$ & $0.00 \%$ & $0.00 \%$ & $43.15 \%$ & $56.66 \%$ & $0.00 \%$ & $0.00 \%$ \\
\hline
\end{tabular}

\begin{tabular}{|cccc|cccc|}
\multicolumn{4}{c}{ C38 } & \multicolumn{5}{c|}{ N41 } \\
\hline \multicolumn{4}{c}{$42.29 \%$} & & \multicolumn{4}{c|}{$57.71 \%$} & $\mathrm{~d}$ \\
$\mathrm{~s}$ & $\mathrm{p}$ & $\mathrm{d}$ & $\mathrm{f}$ & $\mathrm{s}$ & $\mathrm{p}$ & $\mathrm{d}$ & $\mathrm{f}$ \\
$55.80 \%$ & $44.18 \%$ & $0.00 \%$ & $0.00 \%$ & $41.89 \%$ & $57.84 \%$ & $0.00 \%$ & $0.00 \%$ \\
\hline
\end{tabular}

\begin{tabular}{|cccc|cccc|}
\hline \multicolumn{4}{|c|}{$43.21 \%$} & \multicolumn{4}{c|}{$56.79 \%$} \\
$\mathrm{~s}$ & $\mathrm{p}$ & $\mathrm{d}$ & $\mathrm{f}$ & $\mathrm{s}$ & $\mathrm{p}$ & $\mathrm{d}$ & $\mathrm{f}$ \\
$0.00 \%$ & $99.93 \%$ & $0.07 \%$ & $0.00 \%$ & $0.00 \%$ & $99.62 \%$ & $0.38 \%$ & $0.00 \%$ \\
\hline
\end{tabular}

\begin{tabular}{|cccc|cccc|}
\hline \multicolumn{4}{|c|}{$43.04 \%$} & \multicolumn{4}{c|}{$56.96 \%$} \\
$\mathrm{~s}$ & $\mathrm{p}$ & $\mathrm{d}$ & $\mathrm{f}$ & $\mathrm{s}$ & $\mathrm{p}$ & $\mathrm{d}$ & $\mathrm{f}$ \\
$0.89 \%$ & $99.05 \%$ & $0.06 \%$ & $0.00 \%$ & $0.72 \%$ & $98.90 \%$ & $0.27 \%$ & $0.00 \%$ \\
\hline
\end{tabular}

\begin{tabular}{|cccc|cccc|}
\multicolumn{4}{c}{ C39 } & \multicolumn{4}{c|}{$\mathbf{N 4 0}$} \\
\hline \multicolumn{4}{c}{$42.31 \%$} & & \multicolumn{4}{c|}{$57.69 \%$} & $\mathrm{~d}$ \\
$\mathrm{~s}$ & $\mathrm{p}$ & $\mathrm{d}$ & $\mathrm{f}$ & $\mathrm{s}$ & $\mathrm{p}$ & $\mathrm{d}$ & $\mathrm{f}$ \\
$56.58 \%$ & $43.40 \%$ & $0.02 \%$ & $0.00 \%$ & $42.47 \%$ & $57.26 \%$ & $0.27 \%$ & $0.00 \%$ \\
\hline
\end{tabular}

\begin{tabular}{|cccc|cccc|}
\hline \multicolumn{4}{|c|}{$43.57 \%$} & \multicolumn{4}{c|}{$56.43 \%$} \\
$\mathrm{~s}$ & $\mathrm{p}$ & $\mathrm{d}$ & $\mathrm{f}$ & $\mathrm{s}$ & $\mathrm{p}$ & $\mathrm{d}$ & $\mathrm{f}$ \\
$0.06 \%$ & $99.88 \%$ & $0.07 \%$ & $0.00 \%$ & $0.05 \%$ & $99.57 \%$ & $0.38 \%$ & $0.00 \%$ \\
\hline
\end{tabular}

\begin{tabular}{|cccc|cccc|}
\hline \multicolumn{4}{|c|}{$43.47 \%$} & \multicolumn{4}{c|}{$56.63 \%$} \\
$\mathrm{~s}$ & $\mathrm{p}$ & $\mathrm{d}$ & $\mathrm{f}$ & $\mathrm{s}$ & $\mathrm{p}$ & $\mathrm{d}$ & $\mathrm{f}$ \\
$0.09 \%$ & $99.85 \%$ & $0.07 \%$ & $0.00 \%$ & $0.09 \%$ & $99.53 \%$ & $0.38 \%$ & $0.00 \%$ \\
\hline
\end{tabular}

S42 
Isomer 1-O,N (Energy $=-2142.46$ Hartrees $)$

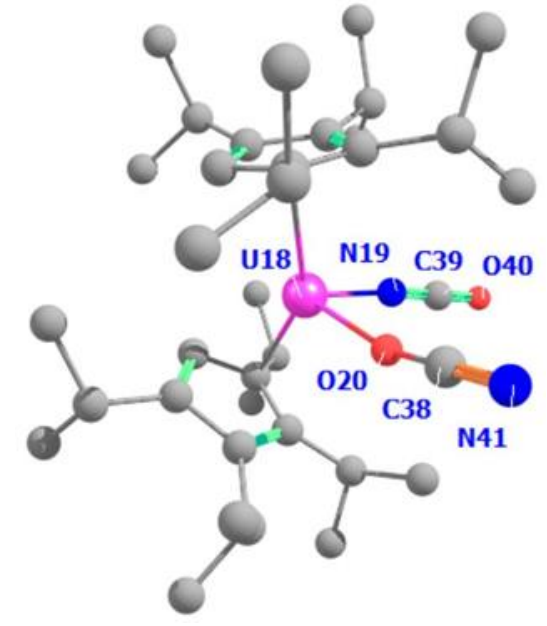

Figure S24. Optimized structure of 1-N3 (uranium: pink, carbon: gray, nitrogen: blue, oxygen: red).

Table S20. Selected calculated metrics for 1-O,N.

\begin{tabular}{lll|ll} 
Bond Coordinate & Distance $(\mathbf{A})$ & WBI & Bond Coordinate & Angle $\left(^{\circ}\right)$ \\
U18-N19 & 2.23 & 0.7 & O20-U18-N19 & 93.5 \\
U18-O20 & 2.19 & 0.6 & U18-N19-C39 & 172.0 \\
N19-C39 & 1.21 & 1.9 & N19-C39-O40 & 179.5 \\
C39-O40 & 1.18 & 1.8 & O20-C38-N41 & 179.5 \\
O20-C38 & 1.26 & 1.2 & U18-O20-C38 & 164.6 \\
C96-N41 & 1.17 & 2.7 & &
\end{tabular}

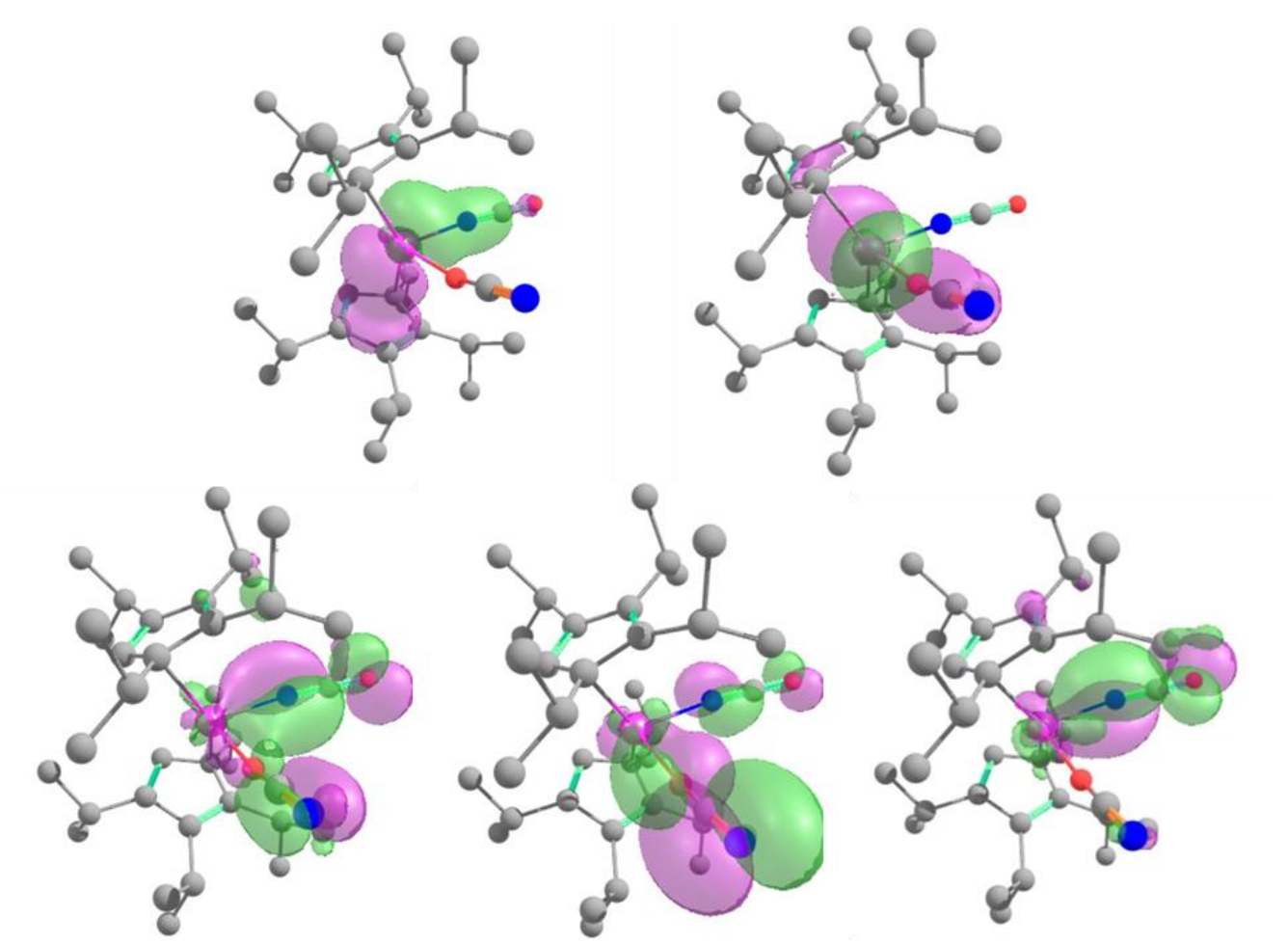

Figure S25. Bonding MOs for 1-O,N (uranium: pink, carbon: gray, nitrogen: blue, oxygen: red). 
Table S21. Relevant natural bonding orbitals in 1-O,N.

\begin{tabular}{|cccc|cccc|}
\multicolumn{4}{c}{ C39 } & \multicolumn{4}{c|}{ O40 } \\
\hline \multicolumn{4}{c}{$34.83 \% .17 \%$} & $\mathrm{~d}$ & $\mathrm{f}$ \\
$\mathrm{s}$ & $\mathrm{p}$ & $\mathrm{d}$ & $\mathrm{f}$ & $\mathrm{s}$ & $\mathrm{p}$ & $\mathrm{d}$ & $0.00 \%$ \\
$46.12 \%$ & $53.78 \%$ & $0.10 \%$ & $0.00 \%$ & $39.88 \%$ & $59.78 \%$ & $0.35 \%$ & $0.00 \%$ \\
\hline
\end{tabular}

\begin{tabular}{|cccc|cccc|}
\multicolumn{4}{c}{ O20 } & \multicolumn{4}{c|}{ C38 } \\
\hline \multicolumn{4}{c}{$67.77 \%$} & & \multicolumn{4}{c|}{$32.23 \%$} & $\mathrm{~d}$ & $\mathrm{f}$ \\
$\mathrm{s}$ & $\mathrm{p}$ & $\mathrm{d}$ & $\mathrm{f}$ & $\mathrm{s}$ & $\mathrm{p}$ & $\mathrm{d}$ & \\
$44.97 \%$ & $54.98 \%$ & $0.04 \%$ & $0.00 \%$ & $43.18 \%$ & $56.63 \%$ & $0.19 \%$ & $0.00 \%$ \\
\hline
\end{tabular}

\begin{tabular}{|cccc|cccc|}
\multicolumn{4}{c}{ N19 } & \multicolumn{4}{c|}{ C39 } \\
\hline \multicolumn{4}{c}{$60.28 \%$} & & \multicolumn{4}{c|}{$39.72 \%$} & $\mathrm{~d}$ \\
$\mathrm{~s}$ & $\mathrm{p}$ & $\mathrm{d}$ & $\mathrm{f}$ & $\mathrm{s}$ & $\mathrm{p}$ & $\mathrm{d}$ & $\mathrm{f}$ \\
$54.56 \%$ & $45.40 \%$ & $0.04 \%$ & $0.00 \%$ & $53.66 \%$ & $46.28 \%$ & $0.05 \%$ & $0.00 \%$ \\
\hline
\end{tabular}

\begin{tabular}{|cccc|cccc|}
\hline \multicolumn{4}{c}{$70.90 \%$} & \multicolumn{4}{c|}{$29.10 \%$} \\
$\mathrm{~s}$ & $\mathrm{p}$ & $\mathrm{d}$ & $\mathrm{f}$ & $\mathrm{s}$ & $\mathrm{p}$ & $\mathrm{d}$ & $\mathrm{f}$ \\
$0.02 \%$ & $99.89 \%$ & $0.09 \%$ & $0.00 \%$ & $0.00 \%$ & $99.72 \%$ & $0.28 \%$ & $0.00 \%$ \\
\hline
\end{tabular}

\begin{tabular}{|cccc|cccc|}
\hline \multicolumn{4}{|c|}{$71.14 \%$} & \multicolumn{4}{c|}{$28.86 \%$} \\
$\mathrm{~s}$ & $\mathrm{p}$ & $\mathrm{d}$ & $\mathrm{f}$ & $\mathrm{s}$ & $\mathrm{p}$ & $\mathrm{d}$ & $\mathrm{f}$ \\
$0.00 \%$ & $99.68 \%$ & $0.08 \%$ & $0.00 \%$ & $0.04 \%$ & $99.68 \%$ & $0.28 \%$ & $0.00 \%$ \\
\hline
\end{tabular}

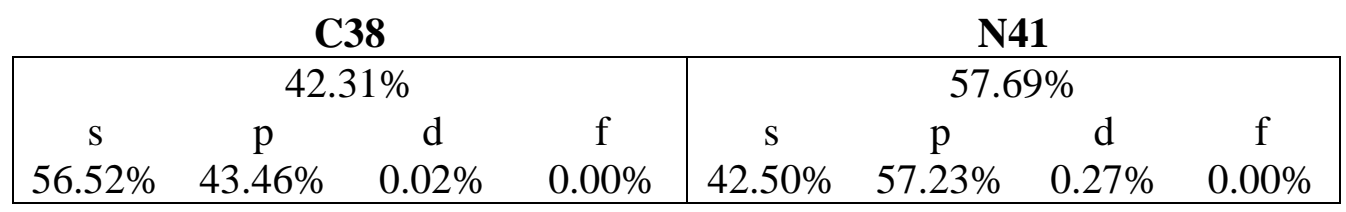

\begin{tabular}{|cccc|cccc|}
\hline \multicolumn{4}{|c|}{$43.47 \%$} & \multicolumn{4}{c|}{$56.53 \%$} \\
$\mathrm{~s}$ & $\mathrm{p}$ & $\mathrm{d}$ & $\mathrm{f}$ & $\mathrm{s}$ & $\mathrm{p}$ & $\mathrm{d}$ & $\mathrm{f}$ \\
$0.04 \%$ & $99.89 \%$ & $0.07 \%$ & $0.00 \%$ & $0.04 \%$ & $99.58 \%$ & $0.38 \%$ & $0.00 \%$ \\
\hline
\end{tabular}

\begin{tabular}{|cccc|cccc|}
\hline \multicolumn{4}{|c|}{$43.23 \%$} & \multicolumn{4}{c|}{$56.77 \%$} \\
$\mathrm{~s}$ & $\mathrm{p}$ & $\mathrm{d}$ & $\mathrm{f}$ & $\mathrm{s}$ & $\mathrm{p}$ & $\mathrm{d}$ & $\mathrm{f}$ \\
$0.10 \%$ & $99.83 \%$ & $0.07 \%$ & $0.00 \%$ & $0.09 \%$ & $99.53 \%$ & $0.38 \%$ & $0.00 \%$ \\
\hline
\end{tabular}

S44 
1-N3 (Energy $=-2134.70$ Hartrees)

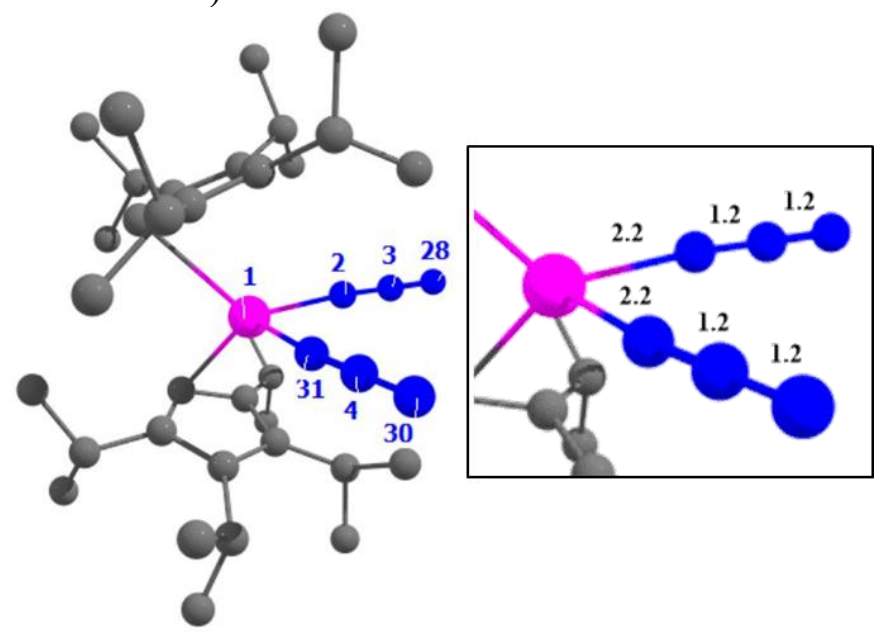

Figure S26. Optimized structure of 1-N3 (atom labels on the left, bond distances in $\AA$ on the right; uranium: pink, carbon: gray, nitrogen: blue).

Table S22. Calculated WBIs for selected bonds in 1-N3.

$\begin{array}{cl}\text { Bond } & \text { WBI } \\ \text { U1-N2 } & 0.8 \\ \mathrm{U} 1-\mathrm{N} 31 & 0.8 \\ \mathrm{~N} 2-\mathrm{N} 3 & 1.6 \\ \mathrm{~N} 31-\mathrm{N} 4 & 1.6 \\ \mathrm{~N} 3-\mathrm{N} 28 & 2.2 \\ \mathrm{~N} 4-\mathrm{N} 30 & 2.2\end{array}$

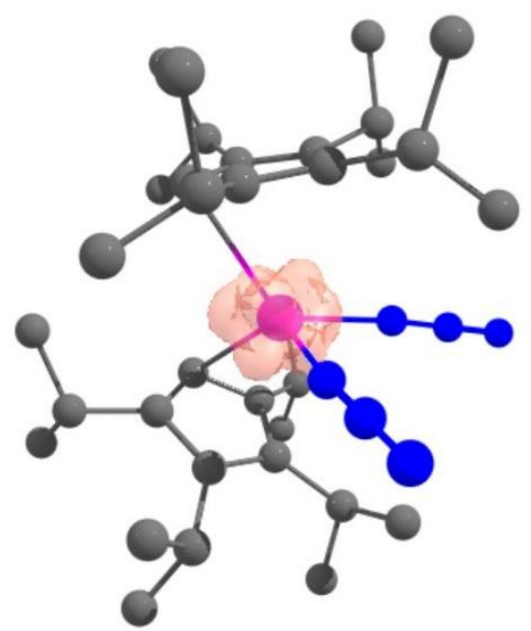

Figure S27. Unpaired spin density plot of 1-N3 demonstrating localization of spin density to the uranium center (uranium: pink, carbon: gray, nitrogen: blue). 
Table S23. Relevant natural bonding orbitals in $\mathbf{1 - N}$
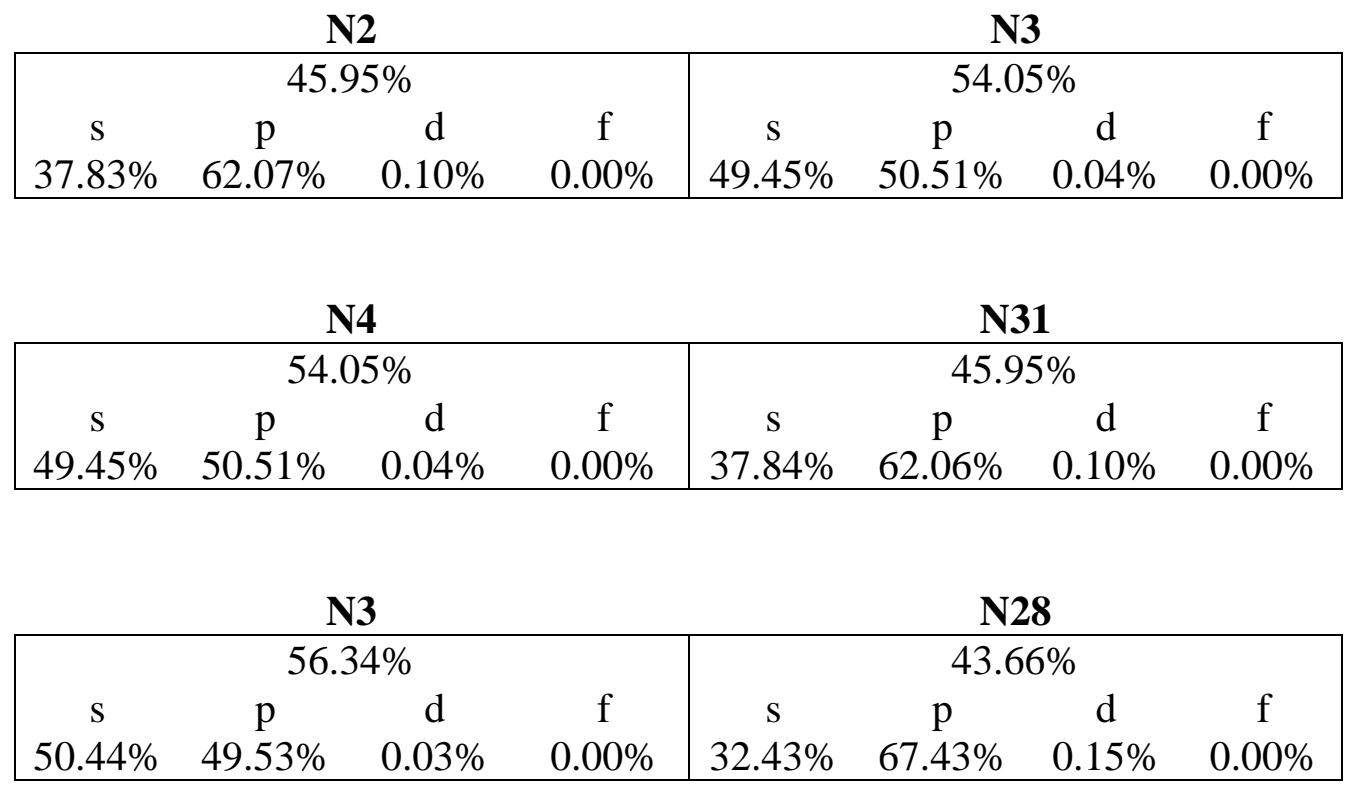

\begin{tabular}{|cccc|cccc|}
\hline \multicolumn{4}{|c|}{$53.04 \%$} & \multicolumn{4}{c|}{$46.96 \%$} \\
$\mathrm{~s}$ & $\mathrm{p}$ & $\mathrm{d}$ & $\mathrm{f}$ & $\mathrm{s}$ & $\mathrm{p}$ & $\mathrm{d}$ & $\mathrm{f}$ \\
$0.00 \%$ & $99.84 \%$ & $0.16 \%$ & $0.00 \%$ & $0.00 \%$ & $99.62 \%$ & $0.38 \%$ & $0.00 \%$ \\
\hline
\end{tabular}

\begin{tabular}{|cccc|cccc|}
\hline \multicolumn{4}{|c|}{$53.39 \%$} & \multicolumn{4}{c|}{$46.51 \%$} \\
$\mathrm{~s}$ & $\mathrm{p}$ & $\mathrm{d}$ & $\mathrm{f}$ & $\mathrm{s}$ & $\mathrm{p}$ & $\mathrm{d}$ & $\mathrm{f}$ \\
$0.0 \%$ & $99.84 \%$ & $0.11 \%$ & $0.00 \%$ & $0.05 \%$ & $99.69 \%$ & $0.25 \%$ & $0.00 \%$ \\
\hline
\end{tabular}

\begin{tabular}{|cccc|cccc|}
\multicolumn{4}{c}{$\mathbf{N 4}$} & \multicolumn{4}{c|}{$\mathbf{N 3 0}$} \\
\hline \multicolumn{4}{c}{$56.34 \%$} & & \multicolumn{4}{c|}{$46 \%$} & $\mathrm{f}$ \\
$\mathrm{s}$ & $\mathrm{p}$ & $\mathrm{d}$ & $\mathrm{f}$ & $\mathrm{s}$ & $\mathrm{p}$ & $\mathrm{d}$ & $\mathrm{f}$ \\
$50.44 \%$ & $49.53 \%$ & $0.03 \%$ & $0.00 \%$ & $32.43 \%$ & $67.43 \%$ & $0.15 \%$ & $0.00 \%$ \\
\hline
\end{tabular}

\begin{tabular}{|cccc|cccc|}
\hline \multicolumn{4}{|c|}{$53.04 \%$} & \multicolumn{4}{c|}{$46.96 \%$} \\
$\mathrm{~s}$ & $\mathrm{p}$ & $\mathrm{d}$ & $\mathrm{f}$ & $\mathrm{s}$ & $\mathrm{p}$ & $\mathrm{d}$ & $\mathrm{f}$ \\
$0.00 \%$ & $99.84 \%$ & $0.16 \%$ & $0.00 \%$ & $0.00 \%$ & $99.62 \%$ & $0.38 \%$ & $0.00 \%$ \\
\hline
\end{tabular}

\begin{tabular}{|cccc|cccc|}
\hline \multicolumn{4}{c}{$53.49 \%$} & \multicolumn{4}{c|}{$46.51 \%$} \\
$\mathrm{~s}$ & $\mathrm{p}$ & $\mathrm{d}$ & $\mathrm{f}$ & $\mathrm{s}$ & $\mathrm{p}$ & $\mathrm{d}$ & $\mathrm{f}$ \\
$0.00 \%$ & $99.84 \%$ & $0.16 \%$ & $0.00 \%$ & $0.00 \%$ & $99.69 \%$ & $0.38 \%$ & $0.00 \%$ \\
\hline
\end{tabular}

S46 


\section{2-NCO}

Isomer 2-N,O (Energy $=-3195.32$ Hartrees)
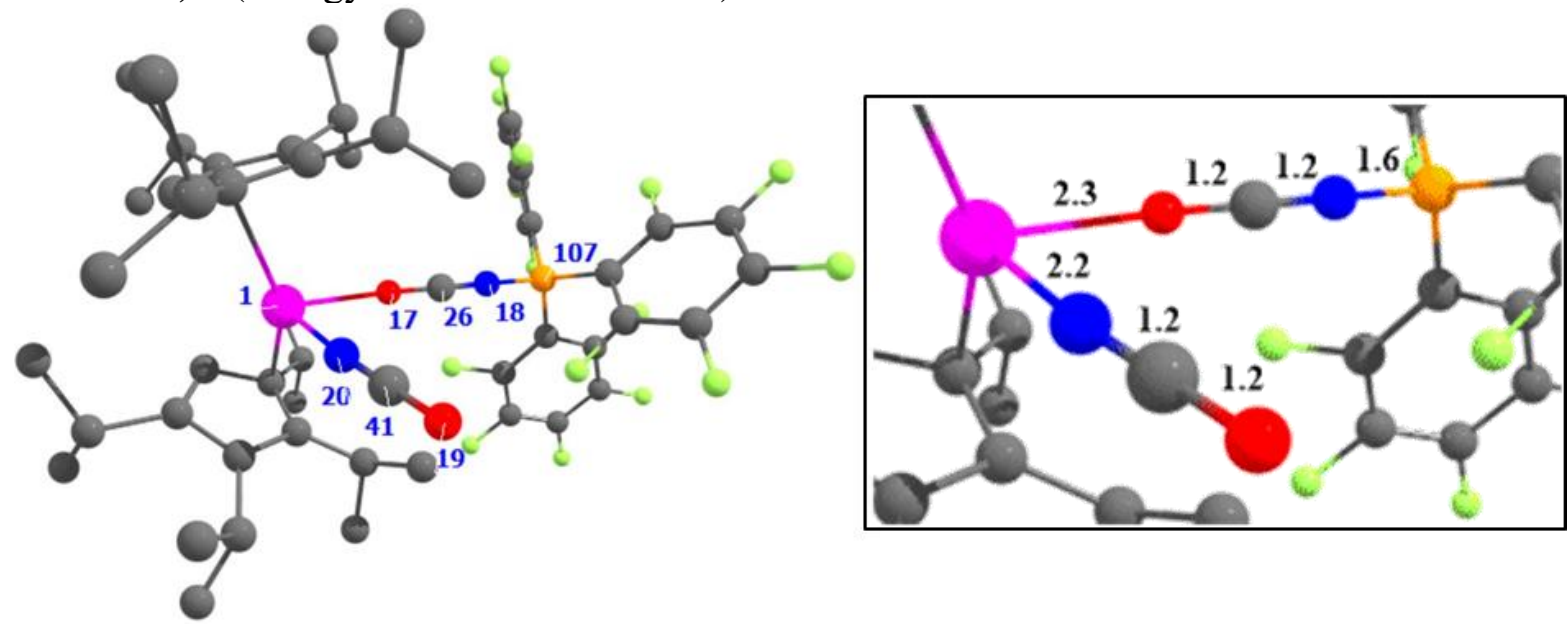

Figure S28. Optimized structure of 2-N,O (atom labels on the left, bond distances in $\AA$ on the right; uranium: pink, carbon: gray, nitrogen: blue, oxygen: red, boron: orange, fluorine: green).

Table S24. Calculated WBIs for selected bonds in 2-N,O.

Bond WBI

U1-O17 0.5

U1-N20 0.8

N20-C41 1.9

C41-O19 1.9

O17-C26 1.4

C26-N18 2.3

N18-B107 0.7

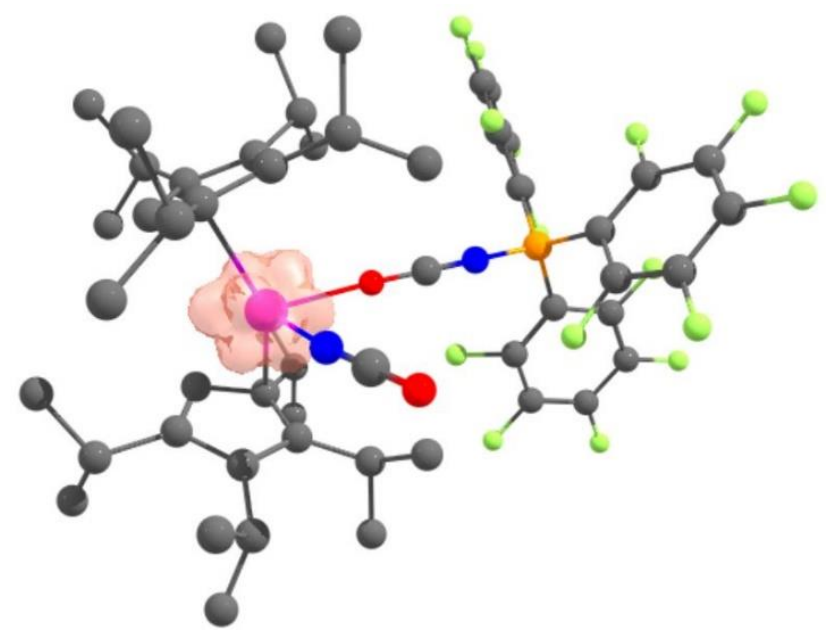

Figure S29. Unpaired spin density plot of 2-N,O demonstrating localization of spin density to the uranium center (uranium: pink, carbon: gray, nitrogen: blue, oxygen: red, boron: orange, fluorine: green). 
Table S25. Relevant natural bonding orbitals in 2-N,O.

\begin{tabular}{|cccc|cccc|}
\multicolumn{4}{c}{ O17 } & \multicolumn{4}{c|}{ C26 } \\
\hline \multicolumn{4}{c}{$63.15 \%$} & & \\
$\mathrm{~s}$ & $\mathrm{p}$ & $\mathrm{d}$ & $\mathrm{f}$ & $\mathrm{s}$ & $\mathrm{p}$ & $\mathrm{d}$ & $\mathrm{f}$ \\
$44.66 \%$ & $55.29 \%$ & $0.04 \%$ & $0.00 \%$ & $44.24 \%$ & $55.57 \%$ & $0.19 \%$ & $0.00 \%$ \\
\hline
\end{tabular}

\begin{tabular}{|cccc|cccc|}
\multicolumn{4}{c}{$\mathbf{N 1 8}$} & \multicolumn{4}{c|}{ C26 } \\
\hline \multicolumn{4}{c}{$61.92 \% .08 \%$} & $\mathrm{p}$ & $\mathrm{d}$ \\
$\mathrm{s}$ & $\mathrm{p}$ & $\mathrm{d}$ & $\mathrm{f}$ & $\mathrm{s}$ & $\mathrm{p}$ & $\mathrm{d}$ & $\mathrm{f}$ \\
$42.32 \%$ & $57.65 \%$ & $0.03 \%$ & $0.00 \%$ & $55.45 \%$ & $44.49 \%$ & $0.06 \%$ & $0.00 \%$ \\
\hline
\end{tabular}

\begin{tabular}{|cccc|cccc|}
\hline \multicolumn{4}{|c|}{$65.73 \%$} & \multicolumn{4}{c|}{$34.27 \%$} \\
$\mathrm{~s}$ & $\mathrm{p}$ & $\mathrm{d}$ & $\mathrm{f}$ & $\mathrm{s}$ & $\mathrm{p}$ & $\mathrm{d}$ & $\mathrm{f}$ \\
$0.03 \%$ & $99.86 \%$ & $0.11 \%$ & $0.00 \%$ & $0.05 \%$ & $99.69 \%$ & $0.25 \%$ & $0.00 \%$ \\
\hline
\end{tabular}

\begin{tabular}{|cccc|cccc|}
\hline \multicolumn{4}{|c|}{$65.94 \%$} & \multicolumn{4}{c|}{$34.06 \%$} \\
$\mathrm{~s}$ & $\mathrm{p}$ & $\mathrm{d}$ & $\mathrm{f}$ & $\mathrm{s}$ & $\mathrm{p}$ & $\mathrm{d}$ & $\mathrm{f}$ \\
$0.03 \%$ & $99.86 \%$ & $0.11 \%$ & $0.00 \%$ & $0.04 \%$ & $99.70 \%$ & $0.26 \%$ & $0.00 \%$ \\
\hline
\end{tabular}

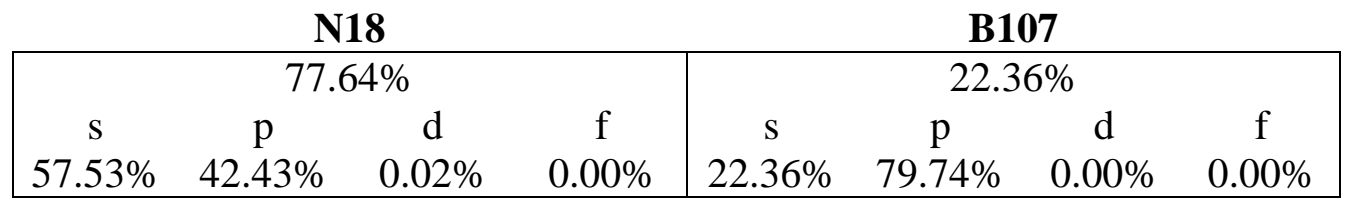

\begin{tabular}{|cccc|cccc|}
\multicolumn{4}{c}{ O19 } & \multicolumn{4}{c|}{ C41 } \\
\hline \multicolumn{4}{c}{$65.21 \%$} & & \multicolumn{4}{c|}{$34.79 \%$} & $\mathrm{~d}$ \\
$\mathrm{~s}$ & $\mathrm{p}$ & $\mathrm{d}$ & $\mathrm{f}$ & $\mathrm{s}$ & $\mathrm{p}$ & $\mathrm{d}$ & $\mathrm{f}$ \\
$54.14 \%$ & $45.82 \%$ & $0.04 \%$ & $0.00 \%$ & $53.62 \%$ & $46.33 \%$ & $0.05 \%$ & $0.00 \%$ \\
\hline
\end{tabular}

\begin{tabular}{|cccc|cccc|}
\multicolumn{4}{c}{ N20 } & \multicolumn{4}{c|}{ C41 } \\
\hline \multicolumn{4}{c}{$60.35 \%$} & & \multicolumn{4}{c|}{$34.79 \%$} & $\mathrm{~d}$ \\
$\mathrm{~s}$ & $\mathrm{p}$ & $\mathrm{d}$ & $\mathrm{f}$ & $\mathrm{s}$ & $\mathrm{p}$ & $\mathrm{d}$ & $\mathrm{f}$ \\
$54.14 \%$ & $45.82 \%$ & $0.04 \%$ & $0.00 \%$ & $53.62 \%$ & $46.33 \%$ & $0.05 \%$ & $0.00 \%$ \\
\hline
\end{tabular}

\begin{tabular}{|cccc|cccc|}
\hline \multicolumn{4}{c}{$71.65 \%$} & \multicolumn{4}{c|}{$28.35 \%$} \\
$\mathrm{~s}$ & $\mathrm{p}$ & $\mathrm{d}$ & $\mathrm{f}$ & $\mathrm{s}$ & $\mathrm{p}$ & $\mathrm{d}$ & $\mathrm{f}$ \\
$0.08 \%$ & $99.84 \%$ & $0.08 \%$ & $0.00 \%$ & $0.02 \%$ & $99.70 \%$ & $0.28 \%$ & $0.00 \%$ \\
\hline
\end{tabular}

\begin{tabular}{|cccc|cccc|}
\hline \multicolumn{4}{|c|}{$71.74 \%$} & \multicolumn{4}{c|}{$28.26 \%$} \\
$\mathrm{~s}$ & $\mathrm{p}$ & $\mathrm{d}$ & $\mathrm{f}$ & $\mathrm{s}$ & $\mathrm{p}$ & $\mathrm{d}$ & $\mathrm{f}$ \\
$0.00 \%$ & $99.92 \%$ & $0.07 \%$ & $0.00 \%$ & $0.01 \%$ & $99.72 \%$ & $0.27 \%$ & $0.00 \%$ \\
\hline
\end{tabular}

S48 
Isomer 2-0,0 (Energy $=-3195.29$ Hartrees)

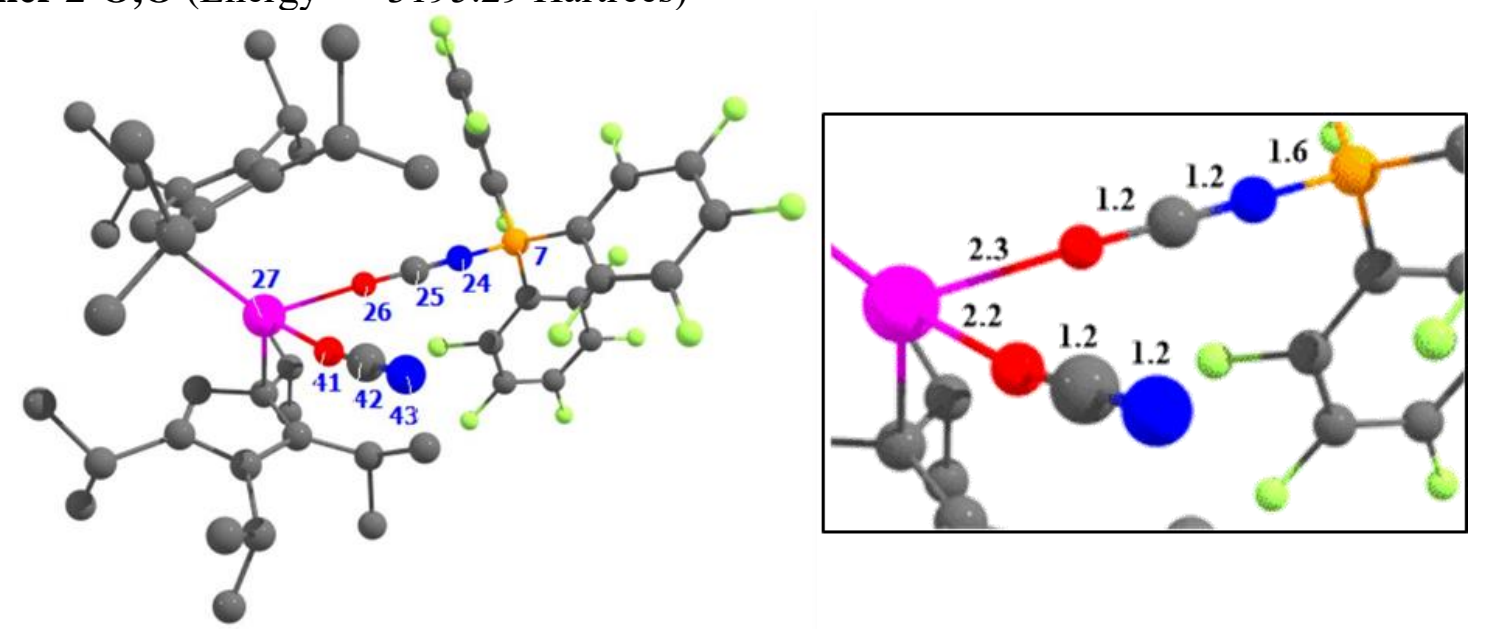

Figure S30. Optimized structure of 2-0,0 (atom labels on the left, bond distances in $\AA$ on the right; uranium: pink, carbon: gray, nitrogen: blue, oxygen: red, boron: orange, fluorine: green).

Table S26. Calculated WBIs for selected bonds in 2-0,0.

$\begin{array}{cl}\text { Bond } & \text { WBI } \\ 27-\mathrm{O} 26 & 0.4 \\ 27-\mathrm{O} 41 & 0.6 \\ 41-\mathrm{C} 42 & 1.1 \\ 42-\mathrm{N} 43 & 2.7 \\ \text { 26-C25 } & 1.4 \\ \text { 25-N24 } & 2.3 \\ \text { 24-B7 } & 0.7\end{array}$

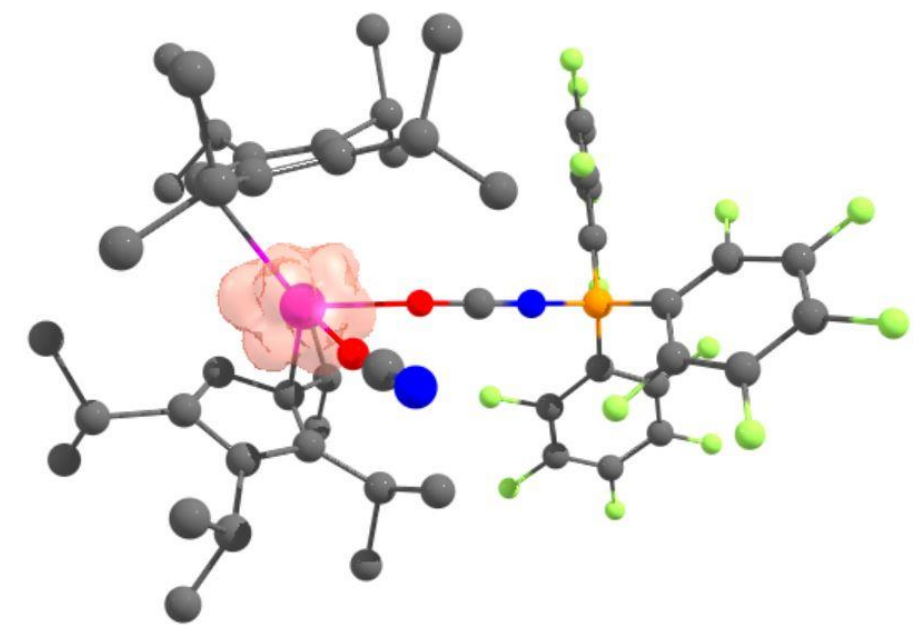

Figure S31. Unpaired spin density plot of 2-O,O demonstrating localization of spin density to the uranium center (uranium: pink, carbon: gray, nitrogen: blue, oxygen: red, boron: orange, fluorine: green). 
Table S27. Relevant natural bonding orbitals in 2-0,0.

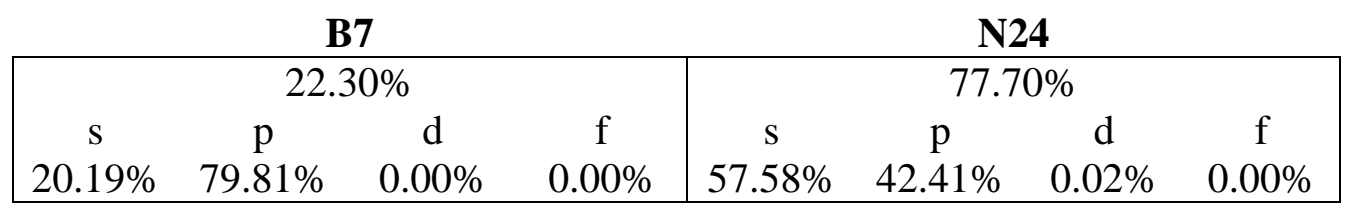

\begin{tabular}{|cccc|cccc|}
\multicolumn{4}{c}{$\mathbf{N 2 4}$} & \multicolumn{4}{c|}{ C25 } \\
\hline \multicolumn{4}{c}{$61.91 \%$} & & \multicolumn{4}{c|}{$389 \%$} & $\mathrm{~d}$ \\
$\mathrm{~s}$ & $\mathrm{p}$ & $\mathrm{d}$ & $\mathrm{f}$ & $\mathrm{s}$ & $\mathrm{p}$ & $\mathrm{d}$ & $\mathrm{f}$ \\
$42.26 \%$ & $57.71 \%$ & $0.03 \%$ & $0.00 \%$ & $55.52 \%$ & $44.42 \%$ & $0.06 \%$ & $0.00 \%$ \\
\hline
\end{tabular}

\begin{tabular}{|cccc|cccc|}
\hline \multicolumn{4}{|c|}{$65.53 \%$} & \multicolumn{4}{c|}{$34.47 \%$} \\
$\mathrm{~s}$ & $\mathrm{p}$ & $\mathrm{d}$ & $\mathrm{f}$ & $\mathrm{s}$ & $\mathrm{p}$ & $\mathrm{d}$ & $\mathrm{f}$ \\
$0.01 \%$ & $99.88 \%$ & $0.11 \%$ & $0.00 \%$ & $0.03 \%$ & $99.72 \%$ & $0.25 \%$ & $0.00 \%$ \\
\hline
\end{tabular}

\begin{tabular}{|cccc|cccc|}
\hline \multicolumn{4}{|c|}{$65.80 \%$} & \multicolumn{4}{c|}{$34.20 \%$} \\
$\mathrm{~s}$ & $\mathrm{p}$ & $\mathrm{d}$ & $\mathrm{f}$ & $\mathrm{s}$ & $\mathrm{p}$ & $\mathrm{d}$ & $\mathrm{f}$ \\
$0.06 \%$ & $99.83 \%$ & $0.11 \%$ & $0.00 \%$ & $0.05 \%$ & $99.69 \%$ & $0.25 \%$ & $0.00 \%$ \\
\hline
\end{tabular}

\begin{tabular}{|cccc|cccc|}
\multicolumn{4}{c}{ C25 } & \multicolumn{5}{c|}{ O26 } \\
\hline \multicolumn{3}{c}{$33.09 \%$} & & \multicolumn{4}{c|}{$61 \%$} \\
$\mathrm{~s}$ & $\mathrm{p}$ & $\mathrm{d}$ & $\mathrm{f}$ & $\mathrm{s}$ & $\mathrm{p}$ & $\mathrm{d}$ & $\mathrm{f}$ \\
$44.18 \%$ & $55.63 \%$ & $0.19 \%$ & $0.00 \%$ & $44.61 \%$ & $55.35 \%$ & $0.04 \%$ & $0.00 \%$ \\
\hline
\end{tabular}

\begin{tabular}{|cccc|cccc|}
\multicolumn{4}{c}{ O41 } & \multicolumn{5}{c|}{$\mathbf{C 4 2}$} \\
\hline \multicolumn{3}{c}{$68.00 \%$} & & \multicolumn{4}{c|}{$32.00 \%$} \\
$\mathrm{~s}$ & $\mathrm{p}$ & $\mathrm{d}$ & $\mathrm{f}$ & $\mathrm{s}$ & $\mathrm{p}$ & $\mathrm{d}$ & $\mathrm{f}$ \\
$44.58 \%$ & $55.38 \%$ & $0.04 \%$ & $0.00 \%$ & $43.00 \%$ & $56.81 \%$ & $0.19 \%$ & $0.00 \%$ \\
\hline
\end{tabular}

\begin{tabular}{|cccc|cccc|}
\multicolumn{4}{c}{$\mathbf{C 4 2}$} & \multicolumn{4}{c|}{$\mathbf{N 4 3}$} \\
\hline \multicolumn{4}{c}{$42.35 \%$} & $\mathrm{p}$ & \multicolumn{4}{c|}{$\mathrm{s} \%$} \\
$\mathrm{~s}$ & $\mathrm{p}$ & $\mathrm{d}$ & $\mathrm{f}$ & $\mathrm{s}$ & $\mathrm{p}$ & $\mathrm{d}$ & $\mathrm{f}$ \\
$56.73 \%$ & $43.25 \%$ & $0.02 \%$ & $0.00 \%$ & $42.37 \%$ & $57.36 \%$ & $0.27 \%$ & $0.00 \%$ \\
\hline
\end{tabular}

\begin{tabular}{|cccc|cccc|}
\hline \multicolumn{4}{|c|}{$44.12 \%$} & \multicolumn{4}{c|}{$55.88 \%$} \\
$\mathrm{~s}$ & $\mathrm{p}$ & $\mathrm{d}$ & $\mathrm{f}$ & $\mathrm{s}$ & $\mathrm{p}$ & $\mathrm{d}$ & $\mathrm{f}$ \\
$0.08 \%$ & $99.86 \%$ & $0.06 \%$ & $0.00 \%$ & $0.07 \%$ & $99.54 \%$ & $0.39 \%$ & $0.00 \%$ \\
\hline
\end{tabular}

\begin{tabular}{|cccc|cccc|}
\hline \multicolumn{4}{|c|}{$44.09 \%$} & \multicolumn{4}{c|}{$55.91 \%$} \\
$\mathrm{~s}$ & $\mathrm{p}$ & $\mathrm{d}$ & $\mathrm{f}$ & $\mathrm{s}$ & $\mathrm{p}$ & $\mathrm{d}$ & $\mathrm{f}$ \\
$0.03 \%$ & $99.90 \%$ & $0.39 \%$ & $0.00 \%$ & $0.03 \%$ & $99.58 \%$ & $0.39 \%$ & $0.00 \%$ \\
\hline
\end{tabular}

S50 
Isomer 2-N,N (Energy $=-3195.31$ Hartrees $)$
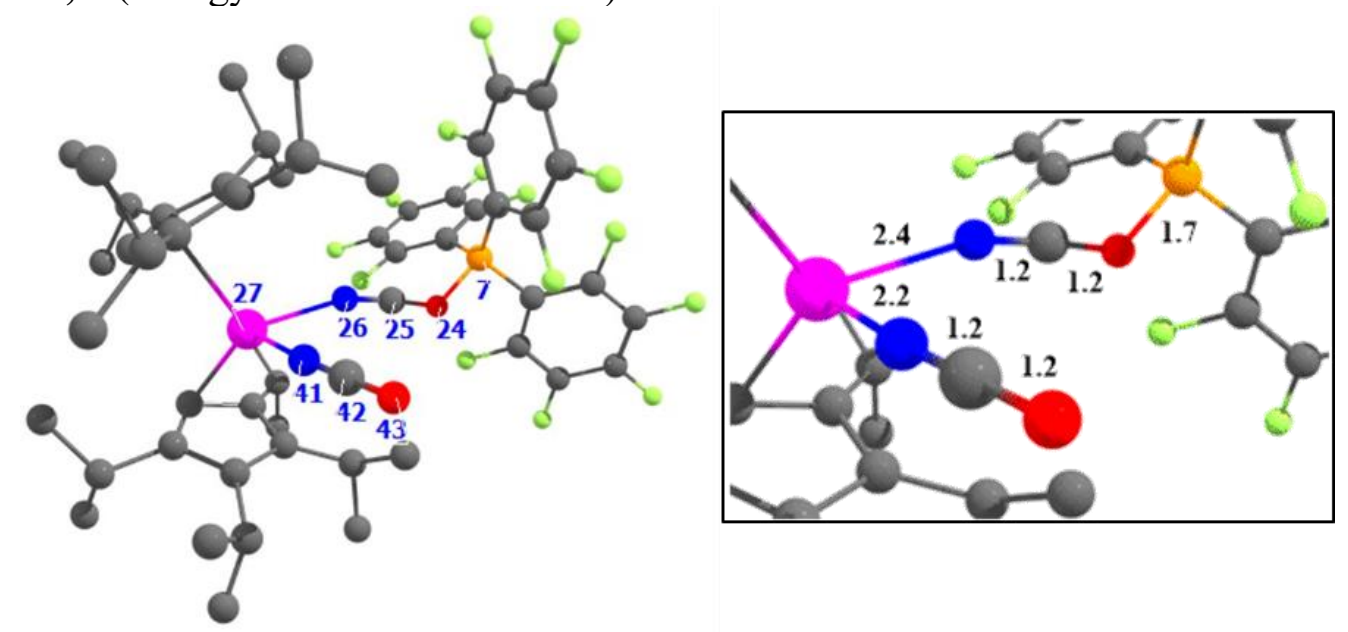

Figure S32. Optimized structure of $\mathbf{2 - N}, \mathbf{N}$ (atom labels on the left, bond distances in $\AA$ on the right; uranium: pink, carbon: gray, nitrogen: blue, oxygen: red, boron: orange, fluorine: green).

Table S28. Calculated WBIs for selected bonds in 2-N,N.

\section{Bond WBI}

$\mathrm{U} 27-\mathrm{N} 41 \quad 0.8$

$\mathrm{U} 27-\mathrm{N} 26 \quad 0.5$

$\mathrm{N} 41-\mathrm{C} 42 \quad 1.9$

$\mathrm{C} 42-\mathrm{O} 43 \quad 1.9$

$\mathrm{N} 26-\mathrm{C} 25 \quad 2.3$

$\mathrm{C} 25-\mathrm{O} 24 \quad 1.4$

$\mathrm{O} 24-\mathrm{B} 7 \quad 0.5$

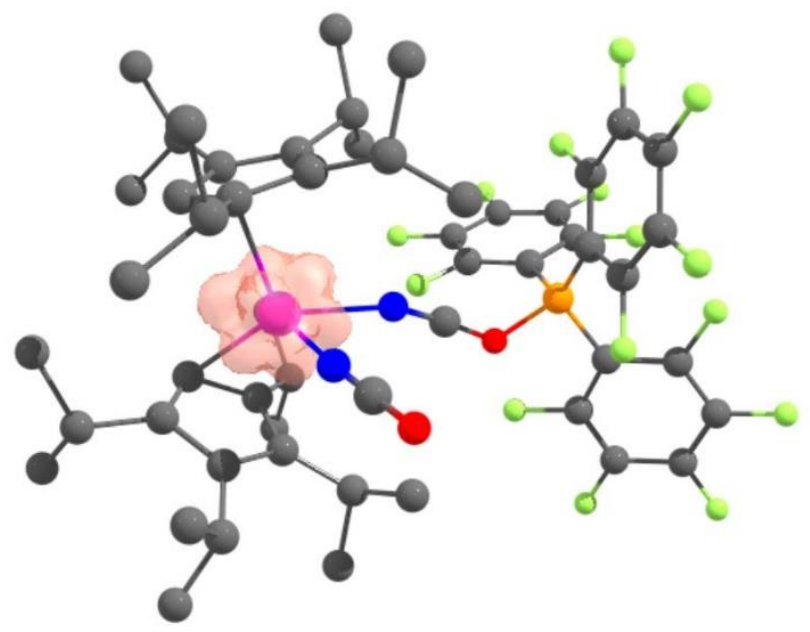

Figure S33. Unpaired spin density plot of $\mathbf{2}-\mathbf{N}, \mathbf{N}$ demonstrating localization of spin density to the uranium center (uranium: pink, carbon: gray, nitrogen: blue, oxygen: red, boron: orange, fluorine: green). 
Table S29. Relevant natural bonding orbitals in $\mathbf{2 - N}, \mathbf{N}$.

\begin{tabular}{|cccc|cccc|}
\multicolumn{4}{c}{$\mathbf{O 2 4}$} & \multicolumn{4}{c|}{ C25 } \\
\hline \multicolumn{4}{c}{$63.20 \%$} & $\mathrm{p}$ & $\mathrm{f}$ \\
$\mathrm{s}$ & $\mathrm{p}$ & $\mathrm{d}$ & $\mathrm{f}$ & $\mathrm{s}$ & $\mathrm{p}$ & $\mathrm{d}$ & $\mathrm{f}$ \\
$41.26 \%$ & $58.69 \%$ & $0.05 \%$ & $0.00 \%$ & $45.29 \%$ & $54.51 \%$ & $0.19 \%$ & $0.00 \%$ \\
\hline
\end{tabular}

\begin{tabular}{|cccc|cccc|}
\multicolumn{4}{c}{ C25 } & \multicolumn{5}{c|}{ N26 } \\
\hline \multicolumn{4}{c}{$39.90 \%$} & & \multicolumn{4}{c|}{$60.10 \%$} & $\mathrm{~d}$ \\
$\mathrm{~s}$ & $\mathrm{p}$ & $\mathrm{d}$ & $\mathrm{f}$ & $\mathrm{s}$ & $\mathrm{p}$ & $\mathrm{d}$ & $\mathrm{f}$ \\
$52.93 \%$ & $47.01 \%$ & $0.06 \%$ & $0.00 \%$ & $52.44 \%$ & $47.52 \%$ & $0.04 \%$ & $0.00 \%$ \\
\hline
\end{tabular}

\begin{tabular}{|cccc|cccc|}
\hline \multicolumn{4}{|c|}{$35.81 \%$} & \multicolumn{4}{c|}{$64.19 \%$} \\
$\mathrm{~s}$ & $\mathrm{p}$ & $\mathrm{d}$ & $\mathrm{f}$ & $\mathrm{s}$ & $\mathrm{p}$ & $\mathrm{d}$ & $\mathrm{f}$ \\
$1.46 \%$ & $98.33 \%$ & $0.21 \%$ & $0.00 \%$ & $1.26 \%$ & $98.60 \%$ & $0.14 \%$ & $0.00 \%$ \\
\hline
\end{tabular}

\begin{tabular}{|cccc|cccc|}
\hline \multicolumn{4}{|c|}{$33.55 \%$} & \multicolumn{4}{c|}{$66.45 \%$} \\
$\mathrm{~s}$ & $\mathrm{p}$ & $\mathrm{d}$ & $\mathrm{f}$ & $\mathrm{s}$ & $\mathrm{p}$ & $\mathrm{d}$ & $\mathrm{f}$ \\
$0.03 \%$ & $99.72 \%$ & $0.25 \%$ & $0.00 \%$ & $0.05 \%$ & $99.82 \%$ & $0.13 \%$ & $0.00 \%$ \\
\hline
\end{tabular}

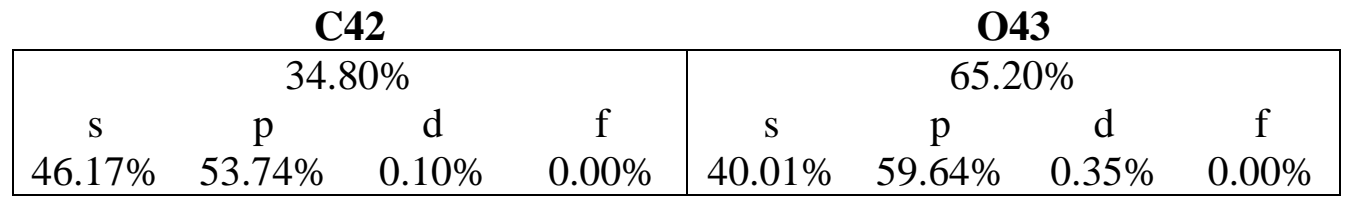

\begin{tabular}{|cccc|cccc|}
\multicolumn{4}{c}{ N41 } & \multicolumn{5}{c|}{$\mathbf{C 4 2}$} \\
\hline \multicolumn{4}{c}{$60.33 \%$} & & \multicolumn{4}{c|}{$39.67 \%$} & $\mathrm{~d}$ & $\mathrm{f}$ \\
$\mathrm{s}$ & $\mathrm{p}$ & $\mathrm{d}$ & $\mathrm{f}$ & $\mathrm{s}$ & $\mathrm{p}$ & $\mathrm{d}$ & \\
$54.38 \%$ & $45.58 \%$ & $0.04 \%$ & $0.00 \%$ & $53.64 \%$ & $46.30 \%$ & $0.05 \%$ & $0.00 \%$ \\
\hline
\end{tabular}

\begin{tabular}{|cccc|cccc|}
\hline \multicolumn{4}{|c|}{$71.36 \%$} & \multicolumn{4}{c|}{$28.64 \%$} \\
$\mathrm{~s}$ & $\mathrm{p}$ & $\mathrm{d}$ & $\mathrm{f}$ & $\mathrm{s}$ & $\mathrm{p}$ & $\mathrm{d}$ & $\mathrm{f}$ \\
$0.06 \%$ & $99.86 \%$ & $0.08 \%$ & $0.00 \%$ & $0.01 \%$ & $99.71 \%$ & $0.28 \%$ & $0.00 \%$ \\
\hline
\end{tabular}

\begin{tabular}{|cccc|cccc|}
\hline \multicolumn{4}{|c|}{$71.60 \%$} & \multicolumn{4}{c|}{$28.40 \%$} \\
$\mathrm{~s}$ & $\mathrm{p}$ & $\mathrm{d}$ & $\mathrm{f}$ & $\mathrm{s}$ & $\mathrm{p}$ & $\mathrm{d}$ & $\mathrm{f}$ \\
$0.00 \%$ & $99.92 \%$ & $0.08 \%$ & $0.00 \%$ & $0.01 \%$ & $99.72 \%$ & $0.27 \%$ & $0.00 \%$ \\
\hline
\end{tabular}

S52 
Isomer 2-O,N (Energy $=-3195.28$ Hartrees)

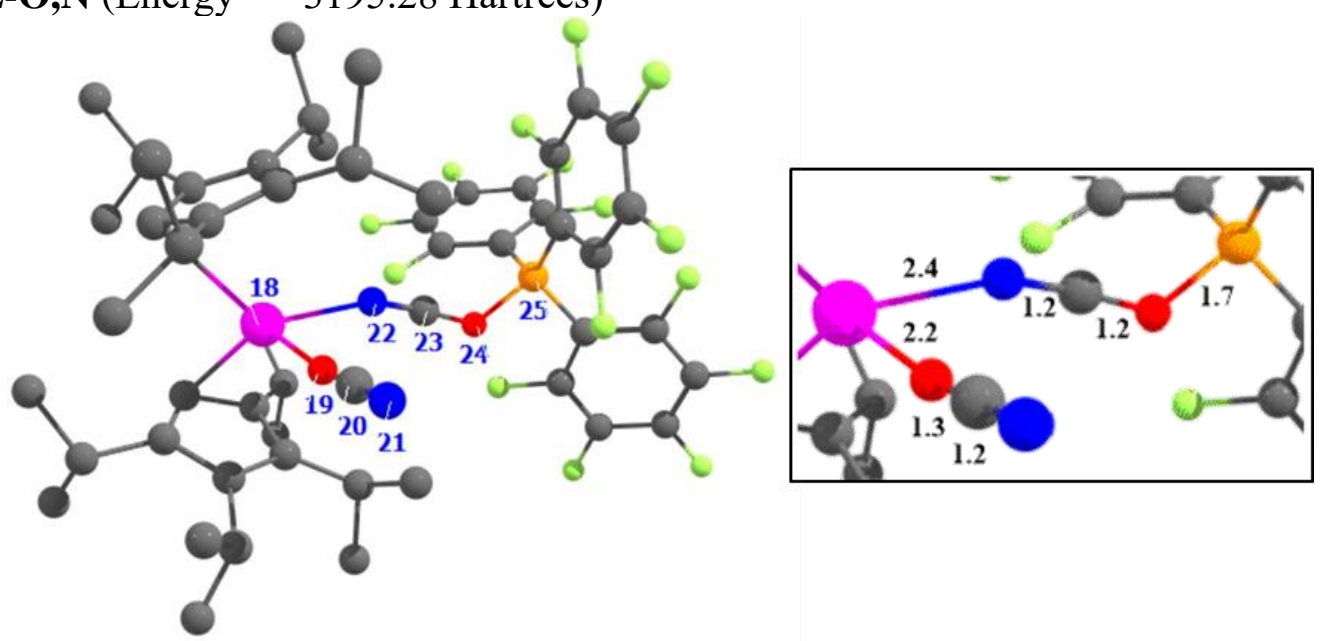

Figure S34. Optimized structure of 2-O,N (atom labels on the left, bond distances in $\AA$ on the right; uranium: pink, carbon: gray, nitrogen: blue, oxygen: red, boron: orange, fluorine: green).

Table S30. Calculated WBIs for selected bonds in 2-O,N.

$\begin{array}{cl}\text { Bond } & \text { WBI } \\ 18-\mathrm{O} 19 & 0.5 \\ 18-\mathrm{N} 22 & 0.8 \\ 22-\mathrm{C} 23 & 1.9 \\ 23-\mathrm{O} 24 & 1.9 \\ 24-\mathrm{B} 25 & 0.7 \\ 19-\mathrm{C} 20 & 2.3 \\ 20-\mathrm{N} 21 & 1.4\end{array}$

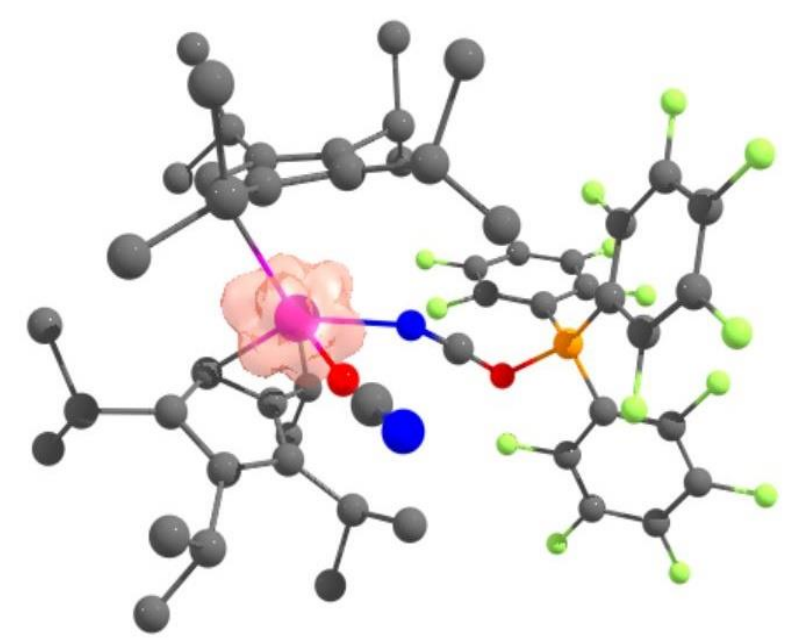

Figure S35. Unpaired spin density plot of $\mathbf{2 - O}, \mathbf{N}$ demonstrating localization of spin density to the uranium center (uranium: pink, carbon: gray, nitrogen: blue, oxygen: red, boron: orange, fluorine: green). 
Table S31. Relevant natural bonding orbitals in $\mathbf{2 - O}, \mathbf{N}$.

\begin{tabular}{|cccc|cccc|}
\multicolumn{4}{c}{ O19 } & \multicolumn{4}{c|}{ C20 } \\
\hline \multicolumn{4}{c}{$67.94 \%$} & & \multicolumn{4}{c|}{} \\
$\mathrm{s}$ & $\mathrm{p}$ & $\mathrm{d}$ & $\mathrm{f}$ & $\mathrm{s}$ & $\mathrm{p}$ & $\mathrm{d}$ & $\mathrm{f}$ \\
$44.89 \%$ & $55.27 \%$ & $0.04 \%$ & $0.00 \%$ & $43.05 \%$ & $56.76 \%$ & $0.19 \%$ & $0.00 \%$ \\
\hline
\end{tabular}

\begin{tabular}{|cccc|cccc|}
\multicolumn{4}{c}{ C20 } & \multicolumn{4}{c|}{ N21 } \\
\hline \multicolumn{4}{c}{$42.35 \%$} & $\mathrm{p}$ & \multicolumn{4}{c|}{$\mathrm{s}$} & $\mathrm{p}$ & $\mathrm{d}$ & $\mathrm{f}$ \\
$\mathrm{s}$ & $\mathrm{p}$ & $\mathrm{d}$ & $\mathrm{f}$ & $\mathrm{s}$ & $\mathrm{p}$ & \\
$56.62 \%$ & $43.36 \%$ & $0.02 \%$ & $0.00 \%$ & $42.37 \%$ & $57.36 \%$ & $0.27 \%$ & $0.00 \%$ \\
\hline
\end{tabular}

\begin{tabular}{|cccc|cccc|}
\hline \multicolumn{4}{|c|}{$44.01 \%$} & \multicolumn{4}{c|}{$55.99 \%$} \\
$\mathrm{~s}$ & $\mathrm{p}$ & $\mathrm{d}$ & $\mathrm{f}$ & $\mathrm{s}$ & $\mathrm{p}$ & $\mathrm{d}$ & $\mathrm{f}$ \\
$0.14 \%$ & $99.80 \%$ & $0.06 \%$ & $0.00 \%$ & $0.12 \%$ & $99.50 \%$ & $0.31 \%$ & $0.00 \%$ \\
\hline
\end{tabular}

\begin{tabular}{|cccc|cccc|}
\hline \multicolumn{4}{|c|}{$43.93 \%$} & \multicolumn{4}{c|}{$56.07 \%$} \\
$\mathrm{~s}$ & $\mathrm{p}$ & $\mathrm{d}$ & $\mathrm{f}$ & $\mathrm{s}$ & $\mathrm{p}$ & $\mathrm{d}$ & $\mathrm{f}$ \\
$0.03 \%$ & $99.91 \%$ & $0.06 \%$ & $0.00 \%$ & $0.03 \%$ & $99.59 \%$ & $0.38 \%$ & $0.00 \%$ \\
\hline
\end{tabular}

\begin{tabular}{|cccc|cccc|}
\multicolumn{4}{c}{ C23 } & \multicolumn{5}{c|}{ O24 } \\
\hline \multicolumn{4}{c}{$33.21 \%$} & & \multicolumn{4}{c|}{$66.79 \%$} & $\mathrm{~d}$ & $\mathrm{f}$ \\
$\mathrm{s}$ & $\mathrm{p}$ & $\mathrm{d}$ & $\mathrm{f}$ & $\mathrm{s}$ & $\mathrm{p}$ & $\mathrm{d}$ & \\
$45.35 \%$ & $54.46 \%$ & $0.19 \%$ & $0.00 \%$ & $41.30 \%$ & $58.65 \%$ & $0.05 \%$ & $0.00 \%$ \\
\hline
\end{tabular}

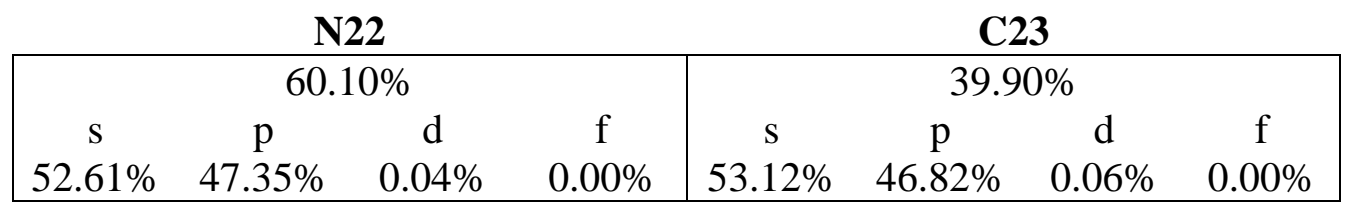

\begin{tabular}{|cccc|cccc|}
\hline \multicolumn{4}{|c|}{$65.76 \%$} & \multicolumn{4}{c|}{$34.24 \%$} \\
$\mathrm{~s}$ & $\mathrm{p}$ & $\mathrm{d}$ & $\mathrm{f}$ & $\mathrm{s}$ & $\mathrm{p}$ & $\mathrm{d}$ & $\mathrm{f}$ \\
$0.21 \%$ & $99.65 \%$ & $0.14 \%$ & $0.00 \%$ & $0.38 \%$ & $99.38 \%$ & $0.24 \%$ & $0.00 \%$ \\
\hline
\end{tabular}

\begin{tabular}{|cccc|cccc|}
\hline \multicolumn{4}{|c|}{$65.31 \%$} & \multicolumn{4}{c|}{$34.69 \%$} \\
$\mathrm{~s}$ & $\mathrm{p}$ & $\mathrm{d}$ & $\mathrm{f}$ & $\mathrm{s}$ & $\mathrm{p}$ & $\mathrm{d}$ & $\mathrm{f}$ \\
$0.82 \%$ & $99.05 \%$ & $0.13 \%$ & $0.00 \%$ & $0.87 \%$ & $98.89 \%$ & $0.23 \%$ & $0.00 \%$ \\
\hline
\end{tabular}

S54 
2-N3 (Energy $=-3187.53$ Hartrees)

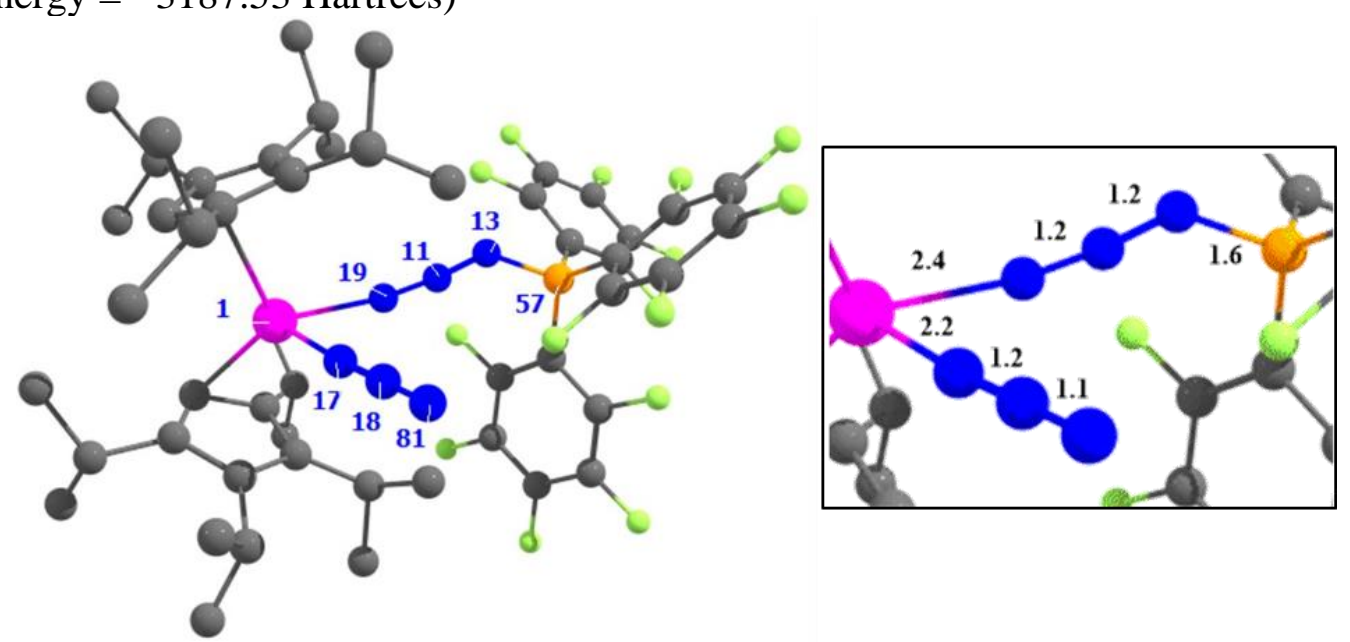

Figure S36. Optimized structure of 2-N3 (atom labels on the left, bond distances in $\AA$ on the right; uranium: pink, carbon: gray, nitrogen: blue, boron: orange, fluorine: green).

Table S32. Calculated WBIs for selected bonds in $\mathbf{2}-\mathbf{N}_{3}$.

$\begin{array}{ll}\text { Bond } & \text { WBI } \\ \text { U1-N19 } & 0.6 \\ \text { U1-N17 } & 0.9 \\ \text { N17-N18 } & 1.6 \\ \text { N18-N81 } & 2.3 \\ \text { N19-N11 } & 1.9 \\ \text { N11-N13 } & 1.9 \\ \text { N13-B57 } & 0.7\end{array}$

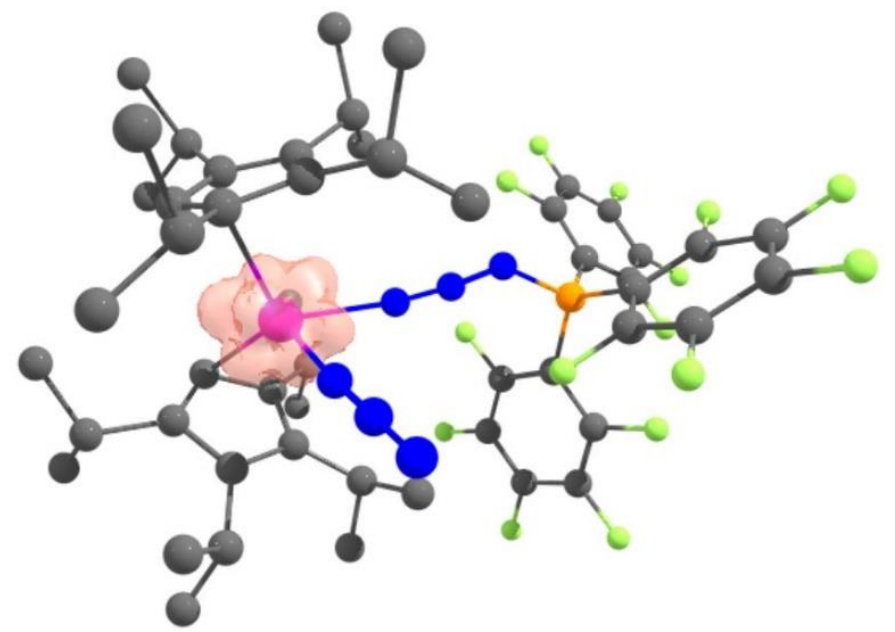

Figure S37. Unpaired spin density plot of 2-O,N demonstrating localization of spin density to the uranium center (uranium: pink, carbon: gray, nitrogen: blue, boron: orange, fluorine: green). 
Table S33. Relevant natural bonding orbitals in $\mathbf{2}-\mathbf{N}_{3}$.

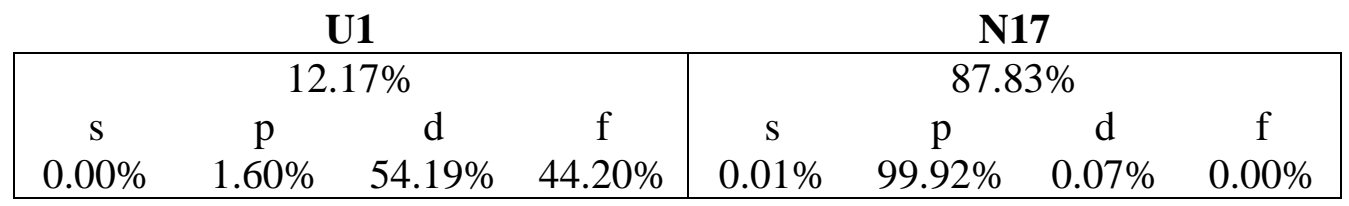

\begin{tabular}{|cccc|cccc|}
\hline \multicolumn{4}{|c|}{$12.56 \%$} & \multicolumn{4}{c|}{$87.44 \%$} \\
$\mathrm{~s}$ & $\mathrm{p}$ & $\mathrm{d}$ & $\mathrm{f}$ & $\mathrm{s}$ & $\mathrm{p}$ & $\mathrm{d}$ & $\mathrm{f}$ \\
$0.01 \%$ & $0.34 \%$ & $33.76 \%$ & $65.88 \%$ & $0.05 \%$ & $99.88 \%$ & $0.07 \%$ & $0.00 \%$ \\
\hline
\end{tabular}

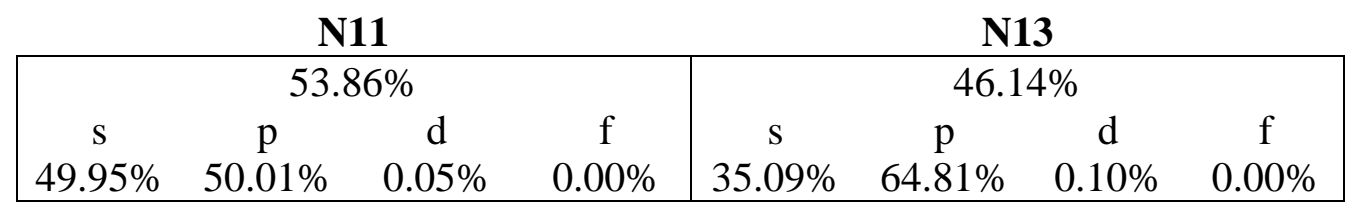

\begin{tabular}{|cccc|cccc|}
\multicolumn{4}{c}{ N11 } & \multicolumn{5}{c|}{ N19 } \\
\hline \multicolumn{4}{c}{$54.27 \%$} \\
$\mathrm{~s}$ & $\mathrm{p}$ & $\mathrm{d}$ & $\mathrm{f}$ & $\mathrm{s}$ & $\mathrm{p}$ & $\mathrm{d}$ & $\mathrm{f}$ \\
$49.87 \%$ & $50.01 \%$ & $0.04 \%$ & $0.00 \%$ & $37.78 \%$ & $62.12 \%$ & $0.04 \%$ & $0.00 \%$ \\
\hline
\end{tabular}

\begin{tabular}{|cccc|cccc|}
\hline \multicolumn{4}{|c|}{$48.17 \%$} & \multicolumn{4}{c|}{$51.83 \%$} \\
$\mathrm{~s}$ & $\mathrm{p}$ & $\mathrm{d}$ & $\mathrm{f}$ & $\mathrm{s}$ & $\mathrm{p}$ & $\mathrm{d}$ & $\mathrm{f}$ \\
$0.09 \%$ & $99.75 \%$ & $0.16 \%$ & $0.00 \%$ & $0.05 \%$ & $99.71 \%$ & $0.24 \%$ & $0.00 \%$ \\
\hline
\end{tabular}

\begin{tabular}{|cccc|cccc|}
\multicolumn{4}{c}{ N13 } & \multicolumn{5}{c|}{ B57 } \\
\hline \multicolumn{4}{c}{$81.62 \%$} & & \multicolumn{4}{c|}{$18.38 \%$} \\
$\mathrm{~s}$ & $\mathrm{p}$ & $\mathrm{d}$ & $\mathrm{f}$ & $\mathrm{s}$ & $\mathrm{p}$ & $\mathrm{d}$ & $\mathrm{f}$ \\
$11.01 \%$ & $89.92 \%$ & $0.07 \%$ & $0.00 \%$ & $19.80 \%$ & $80.20 \%$ & $0.00 \%$ & $0.00 \%$ \\
\hline
\end{tabular}

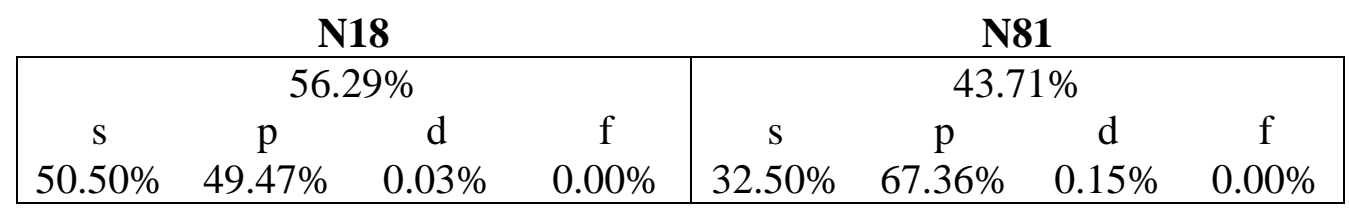

\begin{tabular}{|cccc|cccc|}
\hline \multicolumn{4}{|c|}{$54.53 \%$} & \multicolumn{4}{c|}{$45.47 \%$} \\
$\mathrm{~s}$ & $\mathrm{p}$ & $\mathrm{d}$ & $\mathrm{f}$ & $\mathrm{s}$ & $\mathrm{p}$ & $\mathrm{d}$ & $\mathrm{f}$ \\
$0.00 \%$ & $99.85 \%$ & $0.15 \%$ & $0.00 \%$ & $0.00 \%$ & $99.60 \%$ & $0.40 \%$ & $0.00 \%$ \\
\hline
\end{tabular}

\begin{tabular}{|cccc|cccc|}
\hline \multicolumn{4}{|c|}{$54.56 \%$} & \multicolumn{4}{c|}{$45.44 \%$} \\
$\mathrm{~s}$ & $\mathrm{p}$ & $\mathrm{d}$ & $\mathrm{f}$ & $\mathrm{s}$ & $\mathrm{p}$ & $\mathrm{d}$ & $\mathrm{f}$ \\
$0.00 \%$ & $99.84 \%$ & $0.15 \%$ & $0.00 \%$ & $0.00 \%$ & $99.61 \%$ & $0.39 \%$ & $0.00 \%$ \\
\hline
\end{tabular}

S56 


\begin{tabular}{|cccc|cccc|}
\hline \multicolumn{4}{c}{ N17 } & \multicolumn{4}{c|}{ N18 } \\
\hline \multicolumn{4}{c}{$45.95 \%$} & & \multicolumn{4}{c|}{} & $\mathrm{p} \%$ & $\mathrm{~d}$ & $\mathrm{f}$ \\
$\mathrm{s}$ & $\mathrm{p}$ & $\mathrm{d}$ & $\mathrm{f}$ & $\mathrm{s}$ & $\mathrm{p}$ & $\mathrm{d}$ & \\
$37.82 \%$ & $62.08 \%$ & $0.10 \%$ & $0.00 \%$ & $49.39 \%$ & $50.57 \%$ & $0.04 \%$ & $0.00 \%$ \\
\hline
\end{tabular}

Table S34. Calculated azide vibrational frequencies $\left(\mathrm{cm}^{-1}\right)$ for free $\mathrm{N}_{3}^{-}, \mathbf{1 - N _ { 3 }}$, and $\mathbf{2}-\mathrm{N}_{3}$.

\begin{tabular}{llll} 
Type of vibration & ${\mathbf{F r e e ~} \mathbf{N}^{-}}^{-}$ & $\mathbf{1 - N _ { 3 }}$ & \multicolumn{1}{c}{$\mathbf{2 - N _ { 3 }}$} \\
\hline Wiggle & 646 & 611 & $\begin{array}{l}\text { Uncapped: } 604,608 \\
\text { Capped: } 590,645\end{array}$ \\
Symmetric stretch & 1466 & 1449 & $\begin{array}{l}\text { Uncapped: } 1443 \\
\text { Capped: } 1524\end{array}$ \\
Asymmetric stretch & 2340 & 2265 & $\begin{array}{l}\text { Uncapped: } 2278 \\
\text { Capped: } 2341\end{array}$
\end{tabular}

Table S35. Calculated cyanate/isocyanate vibrational frequencies $\left(\mathrm{cm}^{-1}\right)$ for free $\mathrm{NCO}^{-}, \mathbf{1 - N C O}$, and 2-NCO. ${ }^{\mathrm{a}}$

\begin{tabular}{llll}
\multicolumn{1}{c}{ Type of vibration } & Free NCO $^{-}$ & 1-NCO & \multicolumn{1}{c}{ 2-NCO } \\
\hline Wiggle & 628 & 623 & $\begin{array}{l}\text { Uncapped: } 622,626,636 \\
\text { Both: } 635\end{array}$ \\
Pseudo-symmetric stretch & 1274 & 1417 & $\begin{array}{l}\text { Capped: } 1420 \\
\text { Both: } 1423,1418\end{array}$ \\
Pseudo-asymmetric stretch & 2272 & 2325 & $\begin{array}{l}\text { Uncapped: } 2324 \\
\text { Capped: } 2504\end{array}$
\end{tabular}

a'Both' refers to the fact that the vibrational mode involves both the capped and uncapped fragments.

Table S36. Coordinates for optimized structure of 1-N,N.

$\begin{array}{rrrr}\mathrm{U} & 0.000053 & -0.000138 & -0.186368 \\ \mathrm{O} & 0.395775 & 3.131935 & -3.550437 \\ \mathrm{O} & -0.397822 & -3.129388 & -3.552686 \\ \mathrm{~N} & 0.241105 & 1.618878 & -1.705683 \\ \mathrm{~N} & -0.241211 & -1.619213 & -1.705727 \\ \mathrm{C} & 0.316972 & 2.381064 & -2.641056 \\ \mathrm{C} & -0.318103 & -2.379939 & -2.642209 \\ \mathrm{C} & -1.794782 & 0.914744 & 1.645717 \\ \mathrm{H} & -1.406513 & 1.351931 & 2.557088 \\ \mathrm{C} & -2.078040 & 1.619219 & 0.445934 \\ \mathrm{C} & -2.666696 & 0.683567 & -0.465519 \\ \mathrm{C} & -2.785204 & -0.582892 & 0.211926 \\ \mathrm{C} & -2.258645 & -0.424646 & 1.530707\end{array}$




$\begin{array}{lrrr}\mathrm{C} & -1.957108 & 3.119135 & 0.257304 \\ \mathrm{H} & -1.725750 & 3.317802 & -0.795789 \\ \mathrm{C} & -0.838514 & 3.746461 & 1.091366 \\ \mathrm{H} & -1.043112 & 3.660004 & 2.164296 \\ \mathrm{H} & -0.747231 & 4.813630 & 0.864405 \\ \mathrm{H} & 0.132732 & 3.284831 & 0.887545 \\ \mathrm{C} & -3.292619 & 3.808892 & 0.583695 \\ \mathrm{H} & -4.104000 & 3.440102 & -0.048787 \\ \mathrm{H} & -3.214353 & 4.891618 & 0.433602 \\ \mathrm{H} & -3.574280 & 3.630468 & 1.627407 \\ \mathrm{C} & -3.238080 & 1.094700 & -1.818781 \\ \mathrm{H} & -2.903601 & 2.127755 & -1.970506 \\ \mathrm{C} & -4.777464 & 1.136585 & -1.802263 \\ \mathrm{H} & -5.208184 & 0.130919 & -1.806677 \\ \mathrm{H} & -5.148976 & 1.653883 & -2.693832 \\ \mathrm{H} & -5.162358 & 1.659971 & -0.922004 \\ \mathrm{C} & -2.754338 & 0.319094 & -3.053379 \\ \mathrm{H} & -1.666372 & 0.331252 & -3.138140 \\ \mathrm{H} & -3.166491 & 0.781962 & -3.956951 \\ \mathrm{H} & -3.077045 & -0.725964 & -3.050534 \\ \mathrm{C} & -3.588883 & -1.764090 & -0.319430 \\ \mathrm{H} & -3.739660 & -1.571336 & -1.385193 \\ \mathrm{C} & -4.989320 & -1.813958 & 0.321362 \\ \mathrm{H} & -4.939161 & -2.134587 & 1.366415 \\ \mathrm{H} & -5.621758 & -2.533851 & -0.210505 \\ \mathrm{C} & -5.486415 & -0.840546 & 0.293415 \\ \mathrm{C} & -2.935368 & -3.149653 & -0.230819 \\ \mathrm{H} & -1.950298 & -3.157529 & -0.698016 \\ \mathrm{H} & -3.560721 & -3.884356 & -0.750072 \\ \mathrm{H} & -2.826695 & -3.498414 & 0.800657 \\ \mathrm{C} & -2.508060 & -1.330632 & 2.725835 \\ \mathrm{H} & -2.854418 & -2.298428 & 2.352278 \\ \mathrm{C} & -3.643530 & -0.731886 & 3.578289 \\ \mathrm{H} & -3.323138 & 0.207622 & 4.041545 \\ \mathrm{H} & -3.929270 & -1.421685 & 4.380658 \\ \mathrm{H} & -4.531642 & -0.518333 & 2.977736 \\ \mathrm{H} & -1.293773 & -1.596615 & 3.617089 \\ \mathrm{H} & -0.509759 & -2.131846 & 3.078299 \\ \mathrm{H} & -1.582305 & -2.207913 & 4.479082 \\ \mathrm{H} & -0.663840 & 4.004759 \\ \mathrm{H} & -0.914766 & 1.645583 \\ \mathrm{H} & -1.351896 & 2.557001 \\ \mathrm{H} & -1.619320 & 0.445814 \\ \mathrm{H} & 0.683750 & -0.465803 \\ \mathrm{H} & 0.424609 & 1.530409\end{array}$




$\begin{array}{lrrr}\mathrm{C} & 1.957045 & -3.119212 & 0.257192 \\ \mathrm{H} & 1.725327 & -3.317695 & -0.795850 \\ \mathrm{C} & 3.292502 & -3.809245 & 0.583166 \\ \mathrm{H} & 4.103771 & -3.440644 & -0.049573 \\ \mathrm{H} & 3.213954 & -4.891957 & 0.433104 \\ \mathrm{H} & 3.574538 & -3.630884 & 1.626788 \\ \mathrm{C} & 0.838558 & -3.746310 & 1.091576 \\ \mathrm{H} & 1.043493 & -3.659897 & 2.164446 \\ \mathrm{H} & 0.746945 & -4.813458 & 0.864641 \\ \mathrm{H} & -0.132653 & -3.284463 & 0.888062 \\ \mathrm{C} & 3.237984 & -1.094929 & -1.819106 \\ \mathrm{H} & 2.903438 & -2.127965 & -1.970828 \\ \mathrm{C} & 4.777367 & -1.136936 & -1.802698 \\ \mathrm{H} & 5.208217 & -0.131324 & -1.807129 \\ \mathrm{H} & 5.148769 & -1.654264 & -2.694297 \\ \mathrm{H} & 5.162255 & -1.660377 & -0.922469 \\ \mathrm{C} & 2.754112 & -0.319283 & -3.053622 \\ \mathrm{H} & 1.666154 & -0.331836 & -3.138406 \\ \mathrm{H} & 3.166456 & -0.781889 & -3.957242 \\ \mathrm{H} & 3.076434 & 0.725888 & -3.050592 \\ \mathrm{C} & 3.589200 & 1.763838 & -0.319866 \\ \mathrm{H} & 3.740072 & 1.570973 & -1.385595 \\ \mathrm{C} & 4.989590 & 1.813622 & 0.321029 \\ \mathrm{H} & 4.939416 & 2.134335 & 1.366056 \\ \mathrm{H} & 5.622154 & 2.533393 & -0.210852 \\ \mathrm{H} & 5.486562 & 0.840144 & 0.293187 \\ \mathrm{C} & 2.935780 & 3.149473 & -0.231527 \\ \mathrm{H} & 1.950937 & 3.157467 & -0.699199 \\ \mathrm{H} & 3.561459 & 3.884097 & -0.750504 \\ \mathrm{H} & 2.826662 & 3.498285 & 0.799888 \\ \mathrm{C} & 2.508596 & 1.330711 & 2.725417 \\ \mathrm{H} & 2.855491 & 2.298253 & 2.351705 \\ \mathrm{C} & 3.643590 & 0.731601 & 3.578237 \\ \mathrm{H} & 3.322643 & -0.207656 & 4.041615 \\ \mathrm{H} & 3.929469 & 1.421441 & 4.380518 \\ \mathrm{H} & 4.531738 & 0.517501 & 2.977928 \\ \mathrm{C} & 1.294232 & 1.597513 & 3.616319 \\ \mathrm{H} & 0.510745 & 2.133231 & 3.077254 \\ \mathrm{H} & 1.582880 & 2.208669 & 4.478376 \\ \mathrm{H} & 0.871695 & 0.665050 & 4.003856\end{array}$

Table S37. Coordinates for optimized structure of 1-O,O.
$\begin{array}{llll}\text { C } & 2.648130 & -0.627126 & -0.499319\end{array}$
$\begin{array}{llll}\text { C } & 2.093607 & -1.575639 & 0.421465\end{array}$ 


$\begin{array}{rrrr}\mathrm{C} & 1.809748 & -0.875421 & 1.623630 \\ \mathrm{C} & 2.243511 & 0.474612 & 1.502111 \\ \mathrm{C} & 2.751034 & 0.642288 & 0.176937 \\ \mathrm{C} & 1.993646 & -3.077320 & 0.231604 \\ \mathrm{C} & 0.895699 & -3.723553 & 1.079364 \\ \mathrm{C} & 2.480666 & 1.388507 & 2.693891 \\ \mathrm{C} & 3.646680 & 0.823829 & 3.528326 \\ \mathrm{C} & 3.517217 & 1.842061 & -0.368868 \\ \mathrm{C} & 4.913246 & 1.940091 & 0.275368 \\ \mathrm{C} & 3.192129 & -1.017161 & -1.870109 \\ \mathrm{C} & 4.732078 & -1.023199 & -1.889651 \\ \mathrm{C} & 3.346134 & -3.740303 & 0.544689 \\ \mathrm{C} & 2.656068 & -0.237046 & -3.080836 \\ \mathrm{C} & 2.818393 & 3.206630 & -0.302133 \\ \mathrm{C} & 1.271038 & 1.611923 & 3.603148 \\ \mathrm{U} & 0.000104 & 0.000197 & -0.184759 \\ \mathrm{O} & -0.221545 & -1.570300 & -1.691036 \\ \mathrm{O} & 0.221416 & 1.570233 & -1.691862 \\ \mathrm{C} & -1.809458 & 0.875571 & 1.623846 \\ \mathrm{C} & -2.242916 & -0.474576 & 1.502517 \\ \mathrm{C} & -2.750530 & -0.642545 & 0.177416 \\ \mathrm{C} & -2.647919 & 0.626850 & -0.499000 \\ \mathrm{C} & -2.093588 & 1.575594 & 0.421637 \\ \mathrm{C} & -2.479688 & -1.388542 & 2.694339 \\ \mathrm{C} & -3.646928 & -0.825175 & 3.527937 \\ \mathrm{C} & -1.993984 & 3.077278 & 0.231549 \\ \mathrm{C} & -3.346595 & 3.740037 & 0.544612 \\ \mathrm{C} & -3.192223 & 1.016613 & -1.869733 \\ \mathrm{C} & -4.732183 & 1.022220 & -1.889010 \\ \mathrm{C} & -3.516594 & -1.842475 & -0.368287 \\ \mathrm{C} & -4.912388 & -1.940957 & 0.276398 \\ \mathrm{C} & -0.896166 & 3.723893 & 1.079215 \\ \mathrm{C} & -2.656153 & 0.236587 & -3.080518 \\ \mathrm{C} & -2.817530 & -3.206984 & -0.302190 \\ \mathrm{C} & -1.270506 & -1.610252 & 3.604626 \\ \mathrm{H} & 1.441073 & -1.319592 & 2.539680 \\ \mathrm{H} & 1.760230 & -3.291271 & -0.818128 \\ \mathrm{H} & 1.111858 & -3.637187 & 2.150102 \\ \mathrm{H} & 0.818481 & -4.790264 & 0.847705 \\ \mathrm{H} & -0.088125 & -3.280616 & 0.891099 \\ \mathrm{H} & 4.142458 & -3.356042 & -0.097531 \\ \mathrm{H} & 3.285378 & -4.823351 & 0.392478 \\ \mathrm{H} & 3.635543 & -3.557566 & 1.585569 \\ \mathrm{H} & 2.878036 & -2.055434 & -2.027061 \\ \mathrm{H} & 5.141573 & -0.008749 & -1.886657 \\ \mathrm{H} & 5.091589 & -1.518353 & -2.798019\end{array}$




\begin{tabular}{|c|c|c|c|}
\hline & & & \\
\hline & & & \\
\hline & 376 & 486 & \\
\hline & & & \\
\hline & 0731 & 294 & \\
\hline & & & \\
\hline & 6035 & 7264 & \\
\hline & & & \\
\hline & & & \\
\hline & & & \\
\hline & & & \\
\hline & & & \\
\hline & & & \\
\hline & & & \\
\hline & & & \\
\hline & & & \\
\hline & & & \\
\hline & & & \\
\hline & & & \\
\hline & & & \\
\hline & & & \\
\hline & & & \\
\hline & & & \\
\hline & & & \\
\hline & & & \\
\hline & & & \\
\hline & & & \\
\hline & & & \\
\hline & & & \\
\hline & & & \\
\hline & & & \\
\hline & -3.0 & & \\
\hline & -2.9 & 02 & \\
\hline & -3.6 & & \\
\hline & & & \\
\hline & -5.5 & & \\
\hline & & & \\
\hline & & & \\
\hline & -3.4 & & \\
\hline & -2.6 & -3 & \\
\hline & -2.7 & -2. & \\
\hline & -3.3 & & \\
\hline & -3.9 & -1.5 & \\
\hline & & & \\
\hline & -0.461256 & -2.124068 & 3.082295 \\
\hline & -1.55299 & -2.226205 & 4.46499 \\
\hline
\end{tabular}




$\begin{array}{rrrr}\mathrm{H} & -0.885587 & -0.662533 & 3.994495 \\ \mathrm{C} & 0.013786 & 2.520769 & -2.496855 \\ \mathrm{C} & -0.015536 & -2.520118 & -2.497279 \\ \mathrm{~N} & 0.174624 & -3.410429 & -3.235548 \\ \mathrm{~N} & -0.177967 & 3.411683 & -3.233988\end{array}$

Table S38. Coordinates for optimized structure of 1-O,N.

$\begin{array}{lrrr}\mathrm{C} & 2.665265 & -0.680719 & -0.493611 \\ \mathrm{C} & 2.120653 & -1.573137 & 0.485730 \\ \mathrm{C} & 1.856923 & -0.806030 & 1.651098 \\ \mathrm{C} & 2.294658 & 0.532131 & 1.449371 \\ \mathrm{C} & 2.780750 & 0.623770 & 0.108688 \\ \mathrm{C} & 2.020260 & -3.083152 & 0.387253 \\ \mathrm{C} & 0.925096 & -3.675942 & 1.276409 \\ \mathrm{C} & 2.558631 & 1.509534 & 2.583607 \\ \mathrm{C} & 3.729549 & 0.981568 & 3.434959 \\ \mathrm{C} & 3.541714 & 1.789024 & -0.513878 \\ \mathrm{C} & 4.943627 & 1.928433 & 0.109501 \\ \mathrm{C} & 3.199464 & -1.147857 & -1.843153 \\ \mathrm{C} & 4.739256 & -1.164147 & -1.873916 \\ \mathrm{C} & 3.372256 & -3.731814 & 0.729154 \\ \mathrm{C} & 2.658484 & -0.433920 & -3.091597 \\ \mathrm{C} & 2.838941 & 3.153070 & -0.522452 \\ \mathrm{C} & 1.365327 & 1.803394 & 3.494352 \\ \mathrm{U} & 0.010206 & -0.026135 & -0.185091 \\ \mathrm{~N} & -0.244513 & -1.738733 & -1.586634 \\ \mathrm{O} & 0.201266 & 1.441889 & -1.800930 \\ \mathrm{C} & -1.750626 & 0.988544 & 1.608641 \\ \mathrm{C} & -2.207492 & -0.358678 & 1.578750 \\ \mathrm{C} & -2.752075 & -0.597230 & 0.279512 \\ \mathrm{C} & -2.648340 & 0.627996 & -0.473734 \\ \mathrm{C} & -2.054932 & 1.619653 & 0.374293 \\ \mathrm{C} & -2.432226 & -1.191450 & 2.830716 \\ \mathrm{C} & -3.557788 & -0.545292 & 3.661322 \\ \mathrm{C} & -1.941407 & 3.106402 & 0.093927 \\ \mathrm{C} & -3.282233 & 3.802594 & 0.384763 \\ \mathrm{C} & -3.229786 & 0.956135 & -1.846212 \\ \mathrm{C} & -4.769331 & 0.981390 & -1.823578 \\ \mathrm{C} & -3.552619 & -1.812535 & -0.173139 \\ \mathrm{C} & -4.953231 & -1.821551 & 0.470470 \\ \mathrm{C} & -0.826426 & 3.789846 & 0.888525 \\ \mathrm{C} & -2.742998 & 0.115984 & -3.036953 \\ \mathrm{C} & -2.900341 & -3.189590 & 0.009928 \\ \mathrm{C} & -1.201226 & -1.394069 & 3.715493\end{array}$




\begin{tabular}{|c|c|c|c|}
\hline & & & \\
\hline $\mathrm{H}$ & & & \\
\hline & & 54 & \\
\hline & 0.849204 & 6439 & \\
\hline & -0.057531 & & \\
\hline & 674 & 5195 & 879 \\
\hline & & & \\
\hline & 3.6 & & \\
\hline & & & \\
\hline & & 298 & \\
\hline & & & \\
\hline & 5.1 & & \\
\hline & & & \\
\hline & 3.0 & -0. & \\
\hline & & & \\
\hline & & & \\
\hline & 4.8 & & \\
\hline & & & \\
\hline & 5.4 & & \\
\hline & & & \\
\hline & 3.4 & & \\
\hline & & & \\
\hline & 2.8 & & \\
\hline & 3.4 & & \\
\hline $\mathrm{H}$ & 4.0 & & \\
\hline & 4.6 & & \\
\hline $\mathrm{H}$ & 0.5 & & \\
\hline & 1.6 & & \\
\hline & 0.9 & & \\
\hline & & & \\
\hline & & & \\
\hline $\mathrm{H}$ & -4.0 & & \\
\hline $\mathrm{H}$ & -3.2 & & \\
\hline $\mathrm{H}$ & -3.5 & & \\
\hline & -1.0 & & \\
\hline $\mathrm{H}$ & -0.7 & & \\
\hline & 0.15 & & \\
\hline & -2.9 & & \\
\hline $\mathrm{H}$ & -5.1 & -0.0 & -1 . \\
\hline $\mathrm{H}$ & -5.1 & & -2. \\
\hline $\mathrm{H}$ & -5.1 & 1.5 & -0.9 \\
\hline & -1.6 & & \\
\hline & $-3.1^{\prime}$ & 0.5 & $-3 . C$ \\
\hline & & -0.9 & \\
\hline $\mathrm{H}$ & -3.702510 & -1.6 & -1.24 \\
\hline & -4.901924 & -2.073465 & 1.534192 \\
\hline
\end{tabular}




$\begin{array}{lrrr}\mathrm{H} & -5.583975 & -2.576146 & -0.012917 \\ \mathrm{H} & -5.453377 & -0.853706 & 0.379200 \\ \mathrm{H} & -1.912074 & -3.231001 & -0.448554 \\ \mathrm{H} & -3.523680 & -3.955476 & -0.464408 \\ \mathrm{H} & -2.799747 & -3.470057 & 1.062563 \\ \mathrm{H} & -2.779394 & -2.181690 & 2.523699 \\ \mathrm{H} & -3.232332 & 0.417427 & 4.070295 \\ \mathrm{H} & -3.835908 & -1.190461 & 4.502430 \\ \mathrm{H} & -4.451552 & -0.363284 & 3.058818 \\ \mathrm{H} & -0.429298 & -1.973674 & 3.205201 \\ \mathrm{H} & -1.474442 & -1.935891 & 4.627400 \\ \mathrm{H} & -0.769810 & -0.435710 & 4.021277 \\ \mathrm{C} & -0.009656 & 2.352972 & -2.648330 \\ \mathrm{C} & -0.337917 & -2.560249 & -2.469466 \\ \mathrm{O} & -0.432869 & -3.368855 & -3.325042 \\ \mathrm{~N} & -0.203462 & 3.206643 & -3.428230\end{array}$

Table S39. Coordinates for optimized structure of $\mathbf{1 - N}$.

$\begin{array}{lrrr}\mathrm{U} & 0.000202 & -0.000069 & -0.154025 \\ \mathrm{~N} & 0.241732 & 1.603151 & -1.662966 \\ \mathrm{~N} & 0.286948 & 2.438608 & -2.531437 \\ \mathrm{~N} & -0.296703 & -2.432769 & -2.536559 \\ \mathrm{C} & 2.664187 & -0.657134 & -0.503452 \\ \mathrm{C} & -2.769370 & -0.605264 & 0.188073 \\ \mathrm{C} & 1.840822 & -0.930039 & 1.620682 \\ \mathrm{H} & 1.469675 & -1.383849 & 2.531311 \\ \mathrm{C} & -2.015021 & 3.113814 & 0.215731 \\ \mathrm{H} & -1.769009 & 3.309250 & -0.834872 \\ \mathrm{C} & -0.921461 & 3.763671 & 1.066073 \\ \mathrm{H} & -1.145413 & 3.682742 & 2.135614 \\ \mathrm{H} & -0.840221 & 4.830052 & 0.831989 \\ \mathrm{H} & 0.058962 & 3.311902 & 0.885399 \\ \mathrm{C} & -2.118982 & 1.614859 & 0.416595 \\ \mathrm{C} & 2.119833 & -1.615469 & 0.413443 \\ \mathrm{C} & -2.664153 & 0.657059 & -0.500341 \\ \mathrm{C} & -1.838420 & 0.928684 & 1.623064 \\ \mathrm{H} & -1.466452 & 1.382009 & 2.533614 \\ \mathrm{C} & -2.262508 & -0.427981 & 1.510709 \\ \mathrm{C} & 2.770603 & 0.604645 & 0.185747 \\ \mathrm{C} & 2.877900 & 3.181573 & -0.226287 \\ \mathrm{H} & 1.876236 & 3.183253 & -0.656403 \\ \mathrm{H} & 3.475606 & 3.927053 & -0.762141 \\ \mathrm{H} & 2.802213 & 3.521961 & 0.810796 \\ \mathrm{C} & 3.543692 & 1.804511 & -0.348590\end{array}$




$\begin{array}{cccc}\mathrm{H} & 3.672614 & 1.627446 & -1.420009 \\ \mathrm{~N} & 0.336190 & 3.232924 & -3.359215 \\ \mathrm{C} & 2.265167 & 0.426574 & 1.508834 \\ \mathrm{~N} & -0.353817 & -3.222484 & -3.368211 \\ \mathrm{~N} & -0.242990 & -1.602284 & -1.663802 \\ \mathrm{C} & -2.678135 & 0.271749 & -3.085160 \\ \mathrm{H} & -1.587790 & 0.301506 & -3.135528 \\ \mathrm{H} & -3.071391 & 0.721077 & -4.003804 \\ \mathrm{H} & -2.982770 & -0.778893 & -3.084038 \\ \mathrm{C} & -3.211798 & 1.045436 & -1.870050 \\ \mathrm{H} & -2.896760 & 2.084830 & -2.021335 \\ \mathrm{C} & -3.367340 & 3.785763 & 0.509262 \\ \mathrm{H} & -4.160274 & 3.397273 & -0.134902 \\ \mathrm{H} & -3.303119 & 4.867894 & 0.349213 \\ \mathrm{H} & -3.665989 & 3.613115 & 1.549163 \\ \mathrm{C} & -3.542064 & -1.805202 & -0.346643 \\ \mathrm{H} & -3.670766 & -1.627763 & -1.418029 \\ \mathrm{C} & -2.501487 & -1.333842 & 2.708353 \\ \mathrm{H} & -2.843455 & -2.303035 & 2.334928 \\ \mathrm{C} & -2.875875 & -3.182149 & -0.224630 \\ \mathrm{H} & -1.871398 & -3.181485 & -0.648148 \\ \mathrm{H} & -3.469488 & -3.926371 & -0.766715 \\ \mathrm{H} & -2.806705 & -3.525896 & 0.811837 \\ \mathrm{C} & 2.505533 & 1.331396 & 2.706986 \\ \mathrm{H} & 2.848117 & 2.300608 & 2.334144 \\ \mathrm{C} & -4.955015 & -1.862711 & 0.265300 \\ \mathrm{H} & -4.920747 & -2.153837 & 1.319669 \\ \mathrm{H} & -5.564646 & -2.606867 & -0.259791 \\ \mathrm{H} & -5.466812 & -0.898799 & 0.201251 \\ \mathrm{C} & 4.956576 & 1.861149 & 0.263634 \\ \mathrm{H} & 4.922461 & 2.152522 & 1.317937 \\ \mathrm{H} & 5.566848 & 2.604707 & -0.261553 \\ \mathrm{H} & 5.467613 & 0.896804 & 0.199824 \\ \mathrm{C} & 2.675820 & -0.269295 & -3.087867 \\ \mathrm{H} & 1.585418 & -0.298292 & -3.137393 \\ \mathrm{H} & 3.068093 & -0.717812 & -4.007341 \\ \mathrm{C} & 2.980975 & 0.781196 & -3.085770 \\ \mathrm{H} & 3.368249 & -3.786625 & 0.500607 \\ \mathrm{H} & 3.303327 & -4.868657 & -0.145719 \\ \mathrm{H} & 3.670036 & -3.614619 & 1.539707 \\ \mathrm{H} & -3.741992 & 3.565207 \\ \mathrm{H} & -0.201887 & 4.025060 \\ \mathrm{H} & -3.114257 & 0.211558\end{array}$




$\begin{array}{lrrr}\mathrm{H} & 1.765919 & -3.308654 & -0.838489 \\ \mathrm{C} & 3.210186 & -1.044558 & -1.874068 \\ \mathrm{H} & 2.894335 & -2.083607 & -2.026024 \\ \mathrm{C} & -4.751649 & 1.055123 & -1.894466 \\ \mathrm{H} & -5.161343 & 0.040756 & -1.903623 \\ \mathrm{H} & -5.110161 & 1.559097 & -2.798897 \\ \mathrm{H} & -5.169967 & 1.576326 & -1.028172 \\ \mathrm{C} & -3.636795 & -0.745893 & 3.567546 \\ \mathrm{H} & -3.324744 & 0.197814 & 4.028025 \\ \mathrm{H} & -3.910385 & -1.437389 & 4.372663 \\ \mathrm{H} & -4.530726 & -0.543273 & 2.971541 \\ \mathrm{C} & 1.283997 & 1.593740 & 3.590008 \\ \mathrm{H} & 0.499790 & 2.122642 & 3.044232 \\ \mathrm{H} & 1.562776 & 2.209912 & 4.451828 \\ \mathrm{H} & 0.865279 & 0.659964 & 3.978668 \\ \mathrm{C} & 4.750026 & -1.055030 & -1.899969 \\ \mathrm{H} & 5.160248 & -0.040864 & -1.907967 \\ \mathrm{H} & 5.107443 & -1.557846 & -2.805480 \\ \mathrm{H} & 5.168919 & -1.577701 & -1.034842 \\ \mathrm{C} & 0.924139 & -3.764821 & 1.064618 \\ \mathrm{H} & 1.151256 & -3.684755 & 2.133564 \\ \mathrm{H} & 0.842303 & -4.831020 & 0.829907 \\ \mathrm{H} & -0.056883 & -3.313048 & 0.887296 \\ \mathrm{C} & -1.279412 & -1.595874 & 3.590729 \\ \mathrm{H} & -0.495068 & -2.124044 & 3.044454 \\ \mathrm{H} & -1.557490 & -2.212649 & 4.452344 \\ \mathrm{H} & -0.861119 & -0.662043 & 3.979727\end{array}$

Table S40. Coordinates for optimized structure of $\mathbf{2 - N , N}$.
$\begin{array}{llll}\text { C } & 8.069965 & 10.070397 & 6.983258\end{array}$
$\begin{array}{llll}\text { C } & 7.918024 & 9.680678 & 5.655645\end{array}$
$\begin{array}{llll}\text { C } & 7.747809 & 10.577365 & 4.608686\end{array}$
$\begin{array}{llll}\text { C } & 7.762463 & 11.919991 & 4.989184\end{array}$
$\begin{array}{llll}\text { C } & 7.915488 & 12.357306 & 6.296557\end{array}$
$\begin{array}{llll}\text { C } & 8.068279 & 11.418114 & 7.309713\end{array}$
$\begin{array}{llll}\text { B } & 7.504153 & 10.251264 & 3.038907\end{array}$
$\begin{array}{llll}\text { C } & 8.764385 & 10.494896 & 2.058925\end{array}$
$\begin{array}{llll}\text { C } & 10.028814 & 10.911784 & 2.474229\end{array}$
$\begin{array}{llll}\text { C } & 11.075362 & 11.170234 & 1.592814\end{array}$
$\begin{array}{llll}\text { C } & 10.880710 & 11.000704 & 0.229881\end{array}$
$\begin{array}{llll}\text { C } & 9.643203 & 10.569494 & -0.234150\end{array}$
$\begin{array}{llll}\text { C } & 8.630615 & 10.317701 & 0.680127\end{array}$
$\begin{array}{llll}\text { F } & 10.310137 & 11.084717 & 3.776945\end{array}$
F $\quad 9.447213 \quad 10.399232 \quad-1.545119$ 


\begin{tabular}{|c|c|c|c|}
\hline & 46 & & \\
\hline & 7.962620 & & \\
\hline & 563 & 6 & \\
\hline & 606 & & \\
\hline & 648797 & 2.868390 & 102 \\
\hline & & & \\
\hline & & & \\
\hline & 97 & & \\
\hline & 7.1 & & 2.8 \\
\hline & & & \\
\hline & & & \\
\hline $\mathrm{U}$ & & 4. & \\
\hline $\mathrm{C}$ & & & \\
\hline & & 3.5 & -0. \\
\hline & & & \\
\hline & & & 73 \\
\hline & & & \\
\hline & & & -1.5 \\
\hline & & & \\
\hline & & & \\
\hline & & & \\
\hline & & & 506 \\
\hline & & & 52 \\
\hline & & & 47 \\
\hline & 10 & & 254 \\
\hline & & & \\
\hline & & & -0.5 \\
\hline $\mathrm{O}$ & & & \\
\hline 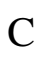 & & & \\
\hline & & & \\
\hline $\mathrm{C}$ & 12 & & 89 \\
\hline & & & \\
\hline C & 7 & 57 & 53 \\
\hline & & & \\
\hline 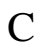 & 2 & 0 & \\
\hline & & & 871 \\
\hline C & & & 3.1 \\
\hline $\mathrm{F}$ & & 80 & 907 \\
\hline & & & \\
\hline & & 079 & 3.443235 \\
\hline & & & 4.07 \\
\hline $\mathrm{F}_{2}$ & & 13.552950 & 409 \\
\hline & & & -1.0 \\
\hline $\mathrm{C}$ & & & 4.083050 \\
\hline & & & 4.400031 \\
\hline & 665720 & 4022601 & 3.764877 \\
\hline
\end{tabular}




$\begin{array}{cccc}\mathrm{C} & 6.539494 & 2.741401 & 3.109547 \\ \mathrm{C} & 7.754285 & 2.021864 & 3.330756 \\ \mathrm{C} & 5.580615 & 5.080322 & 3.990061 \\ \mathrm{C} & 4.505113 & 4.592563 & 4.979243 \\ \mathrm{C} & 5.236102 & 2.187605 & 2.546908 \\ \mathrm{C} & 4.559558 & 1.236713 & 3.553781 \\ \mathrm{C} & 8.437602 & 5.129056 & 5.370696 \\ \mathrm{C} & 9.961660 & 5.261928 & 5.393966 \\ \mathrm{C} & 7.965278 & 0.520998 & 3.206559 \\ \mathrm{C} & 7.801107 & -0.114408 & 4.601318 \\ \mathrm{C} & 7.921757 & 4.839714 & 6.790026 \\ \mathrm{C} & 5.305463 & 1.508505 & 1.173421 \\ \mathrm{C} & 9.306150 & 0.087455 & 2.611480 \\ \mathrm{C} & 4.895207 & 5.682864 & 2.752827 \\ \mathrm{H} & 8.038012 & 6.099416 & 5.065484 \\ \mathrm{H} & 10.713862 & 1.907708 & 0.849306 \\ \mathrm{H} & 4.567645 & 3.044404 & 2.427712 \\ \mathrm{H} & 9.584698 & 2.572020 & 4.462750 \\ \mathrm{H} & 8.275987 & 3.293737 & -1.856907 \\ \mathrm{H} & 5.756870 & 2.159828 & 0.424695 \\ \mathrm{H} & 4.293672 & 1.259833 & 0.835050 \\ \mathrm{H} & 5.871152 & 0.572617 & 1.195849 \\ \mathrm{H} & 7.177571 & 0.115408 & 2.565762 \\ \mathrm{H} & 9.411974 & 0.412191 & 1.574906 \\ \mathrm{H} & 9.389520 & -1.004300 & 2.627355 \\ \mathrm{H} & 10.147261 & 0.486158 & 3.187758 \\ \mathrm{H} & 5.611184 & 6.057630 & 2.018750 \\ \mathrm{H} & 4.266447 & 6.522142 & 3.066431 \\ \mathrm{H} & 4.246262 & 4.967207 & 2.239898 \\ \mathrm{H} & 6.830039 & 4.813614 & 6.825619 \\ \mathrm{H} & 8.260524 & 5.617212 & 7.482884 \\ \mathrm{H} & 8.294384 & 3.874092 & 7.149776 \\ \mathrm{H} & 6.093013 & 5.914627 & 4.483271 \\ \mathrm{H} & 9.451686 & 0.692571 & -0.759617 \\ \mathrm{H} & 8.051448 & 0.867397 & -1.815412 \\ \mathrm{H} & 7.970348 & 1.462848 & -0.157273 \\ \mathrm{H} & 10.656791 & 7.221384 & 2.675964 \\ \mathrm{H} & 12.120656 & 8.134941 & 2.286245 \\ \mathrm{H} & 12.256538 & 6.519878 & 2.964488 \\ \mathrm{H} & 8.615532 & 0.196483 & 5.264366 \\ \mathrm{H} & 7.824678 & -1.207505 & 4.530007 \\ \mathrm{H} & 6.859876 & 0.177897 & 5.073639 \\ \mathrm{H} & 10.438641 & 4.355833 & 5.783500 \\ \mathrm{H} & 10.256597 & 6.090490 & 6.044956 \\ \mathrm{H} & 10.375307 & 5.469231 & 4.400270 \\ \mathrm{H} & 10.400473 & 3.456199 & -3.156577\end{array}$




$\begin{array}{cccc}\mathrm{H} & 9.552368 & 1.945508 & -3.513923 \\ \mathrm{H} & 10.961711 & 1.940252 & -2.438335 \\ \mathrm{H} & 11.091729 & 7.299928 & 0.248294 \\ \mathrm{H} & 7.851248 & 6.756137 & -0.459475 \\ \mathrm{H} & 8.359206 & 7.627164 & -1.909085 \\ \mathrm{H} & 9.309298 & 7.746289 & -0.431409 \\ \mathrm{H} & 12.906582 & 4.703533 & 2.536397 \\ \mathrm{H} & 8.867092 & 5.243350 & -2.119415 \\ \mathrm{H} & 5.090457 & 0.282441 & 3.621103 \\ \mathrm{H} & 3.535561 & 1.018061 & 3.231515 \\ \mathrm{H} & 4.513754 & 1.666456 & 4.557866 \\ \mathrm{H} & 3.842883 & 3.853713 & 4.518707 \\ \mathrm{H} & 3.883448 & 5.435743 & 5.298548 \\ \mathrm{H} & 4.944430 & 4.138505 & 5.871925 \\ \mathrm{H} & 11.598111 & 3.355817 & 4.102379 \\ \mathrm{H} & 13.212855 & 2.669028 & 3.931008 \\ \mathrm{H} & 11.850070 & 1.896730 & 3.120321 \\ \mathrm{H} & 13.377069 & 2.099991 & 1.001677 \\ \mathrm{H} & 14.582100 & 3.026970 & 1.908708 \\ \mathrm{H} & 13.870842 & 3.696856 & 0.428863 \\ \mathrm{H} & 13.780591 & 6.009445 & 0.899447 \\ \mathrm{H} & 13.478381 & 7.612571 & 0.231095 \\ \mathrm{H} & 13.128617 & 6.171246 & -0.736607 \\ \mathrm{H} & 11.312970 & 7.070241 & -1.991999 \\ \mathrm{H} & 10.232261 & 6.787571 & -3.354158 \\ \mathrm{H} & 11.298981 & 5.491897 & -2.786481\end{array}$

Table S41. Coordinates for optimized structure of 2-O,O.
$\begin{array}{llll}\text { C } & 4.569841 & -2.311107 & -2.931829\end{array}$
$\begin{array}{llll}\text { C } & 3.776599 & -1.517664 & -2.127722\end{array}$
$\begin{array}{llll}\text { C } & 4.275865 & -0.579319 & -1.240893\end{array}$
$\begin{array}{llll}\text { C } & 5.662788 & -0.486146 & -1.243027\end{array}$
$\begin{array}{llll}\text { C } & 6.495001 & -1.245752 & -2.027632\end{array}$
$\begin{array}{llll}\text { C } & 5.945399 & -2.190243 & -2.864251\end{array}$
$\begin{array}{llll}\text { B } & 3.460262 & 0.207778 & -0.060272\end{array}$
$\begin{array}{llll}\text { C } & 3.494438 & 1.848327 & -0.129191\end{array}$
$\begin{array}{llll}\text { C } & 3.979139 & 2.624956 & -1.164542\end{array}$
$\begin{array}{llll}\text { C } & 3.814071 & 3.997091 & -1.236259\end{array}$
$\begin{array}{llll}\text { C } & 3.134996 & 4.666127 & -0.240434\end{array}$
$\begin{array}{llll}\text { C } & 2.639491 & 3.940628 & 0.825473\end{array}$
C $2.833766 \quad 2.575001 \quad 0.853862$
F $\quad 4.654169 \quad 2.068730 \quad-2.189978$
F $\quad 1.984863 \quad 4.574020 \quad 1.814425$
F $\quad 2.964799 \quad 5.990236 \quad-0.297626$ 


$\begin{array}{cccc}\text { F } & 2.448055 & -1.740750 & -2.232222 \\ \text { F } & 2.325501 & 1.921183 & 1.933168 \\ \text { F } & 4.314172 & 4.693959 & -2.275570 \\ \text { F } & 6.251756 & 0.397779 & -0.407191 \\ \text { F } & 7.830945 & -1.100221 & -1.977266 \\ \text { F } & 6.738596 & -2.988698 & -3.599598 \\ \text { F } & 4.023630 & -3.223430 & -3.752145 \\ \text { N } & 1.927169 & -0.091872 & -0.140049 \\ \text { C } & 0.780937 & 0.030984 & -0.110693 \\ \text { O } & -0.435611 & 0.155020 & -0.090046 \\ \text { U } & -2.740579 & -0.117324 & 0.01193 \\ \text { C } & -4.597518 & 1.772220 & -0.512380 \\ \text { C } & -4.225956 & 1.978028 & 0.833117 \\ \text { C } & -2.891576 & 2.495313 & 0.821296 \\ \text { C } & -2.473163 & 2.634018 & -0.540577 \\ \text { C } & -3.549049 & 2.179059 & -1.370879 \\ \text { C } & -5.104864 & 1.742026 & 2.045039 \\ \text { C } & -5.750649 & 3.052207 & 2.506286 \\ \text { C } & -1.208296 & 3.364461 & -0.978308 \\ \text { C } & -1.561474 & 4.807772 & -1.409865 \\ \text { C } & -3.706359 & 2.340949 & -2.872869 \\ \text { C } & -4.600910 & 3.558253 & -3.148328 \\ \text { C } & -2.195915 & 2.962264 & 2.091325 \\ \text { C } & -2.187456 & 4.484739 & 2.162242 \\ \text { O } & -2.708537 & -0.412295 & 2.232175 \\ \text { C } & -2.500534 & -0.737982 & 3.352917 \\ \text { N } & -2.296411 & -1.094782 & 4.470810 \\ \text { C } & -6.154434 & 0.667163 & 1.784270 \\ \text { C } & -0.371190 & 2.748361 & -2.089204 \\ \text { C } & -4.381327 & 1.182318 & -3.586612 \\ \text { C } & 4.015023 & -0.452454 & 1.335954 \\ \text { C } & 4.802145 & 0.164794 & 2.295460 \\ \text { C } & 5.337126 & -0.490781 & 3.388676 \\ \text { C } & 5.103958 & -1.837017 & 3.555939 \\ \text { C } & 4.343671 & -2.511547 & 2.625505 \\ \text { C } & 3.816753 & -1.811496 & 1.563342 \\ \text { F } & 5.116178 & 1.473505 & 2.189132 \\ \text { F } & 5.621276 & -2.487576 & 4.610893 \\ \text { F } & 4.127683 & -3.830696 & 2.757886 \\ \text { F } & 3.080194 & -2.536330 & 0.681342 \\ \text { F } & 6.073379 & 0.180968 & 4.288309 \\ \text { C } & -0.776332 & 2.429122 & 2.326767 \\ \text { C } & -3.316826 & -1.986841 & -1.855749 \\ \text { C } & -1.957416 & -2.186551 & -1.536178 \\ \text { C } & -1.909933 & -2.698117 & -0.204629 \\ \text { C } & -3.264970 & -2.855711 & 0.263160\end{array}$




$\begin{array}{rrrr}\mathrm{C} & -4.131113 & -2.402726 & -0.773619 \\ \mathrm{C} & -0.601951 & -3.162614 & 0.447860 \\ \mathrm{C} & -0.539463 & -4.687027 & 0.507402 \\ \mathrm{C} & -3.635797 & -3.625374 & 1.517333 \\ \mathrm{C} & -3.967860 & -5.082068 & 1.144172 \\ \mathrm{C} & -0.785371 & -1.977980 & -2.484894 \\ \mathrm{C} & -1.099974 & -0.974925 & -3.589287 \\ \mathrm{C} & -5.638949 & -2.595542 & -0.887806 \\ \mathrm{C} & -5.926057 & -3.885324 & -1.670728 \\ \mathrm{C} & -0.358237 & -3.317529 & -3.082309 \\ \mathrm{C} & -4.772032 & -3.065134 & 2.383395 \\ \mathrm{C} & -6.361192 & -1.443818 & -1.574484 \\ \mathrm{C} & -0.253310 & -2.598764 & 1.829534 \\ \mathrm{H} & -0.004762 & -1.612748 & -1.944853 \\ \mathrm{H} & -5.450933 & 1.401808 & -0.806977 \\ \mathrm{H} & -2.805217 & -3.653379 & 2.104021 \\ \mathrm{H} & -3.646707 & -1.617094 & -2.696106 \\ \mathrm{H} & -4.508031 & 1.410754 & 2.798666 \\ \mathrm{H} & -4.637068 & -2.084197 & 2.519157 \\ \mathrm{H} & -4.771923 & -3.526831 & 3.270904 \\ \mathrm{H} & -5.647660 & -3.220901 & 1.926423 \\ \mathrm{H} & -6.020501 & -2.699253 & 0.049035 \\ \mathrm{H} & -6.234881 & -0.604319 & -1.046070 \\ \mathrm{H} & -7.337368 & -1.653548 & -1.637320 \\ \mathrm{H} & -5.988728 & -1.316691 & -2.494760 \\ \mathrm{H} & -0.291938 & -1.598666 & 1.805350 \\ \mathrm{H} & 0.670349 & -2.888197 & 2.084379 \\ \mathrm{H} & -0.907310 & -2.936767 & 2.507337 \\ \mathrm{H} & -0.043060 & -3.925423 & -2.352610 \\ \mathrm{H} & 0.385558 & -3.169672 & -3.734784 \\ \mathrm{H} & -1.136707 & -3.737570 & -3.551117 \\ \mathrm{H} & 0.146245 & -2.871705 & -0.176657 \\ \mathrm{H} & -6.810459 & 0.996850 & 1.102918 \\ \mathrm{H} & -6.636577 & 0.456907 & 2.635246 \\ \mathrm{H} & -5.709206 & -0.161963 & 1.442072 \\ \mathrm{H} & -0.167068 & 1.794873 & -1.865192 \\ \mathrm{H} & 0.485372 & 3.258595 & -2.183425 \\ \mathrm{H} & -0.877879 & 2.787723 & -2.951401 \\ \mathrm{H} & -5.611558 & -3.779227 & -2.615119 \\ \mathrm{H} & -6.909850 & -4.067662 & -1.666162 \\ \mathrm{H} & -5.444516 & -4.651710 & -1.245171 \\ \mathrm{H} & -1.802655 & -1.352528 & -4.194347 \\ \mathrm{H} & -0.271173 & -0.791711 & -4.118952 \\ \mathrm{H} & -1.433135 & -0.122382 & -3.185139 \\ \mathrm{H} & -5.037751 & 3.703794 & 2.767326 \\ \mathrm{H} & -6.342168 & 2.874773 & 3.293130\end{array}$




$\begin{array}{lrrr}\mathrm{H} & -6.296472 & 3.438204 & 1.760289 \\ \mathrm{H} & -0.609315 & 3.437955 & -0.161822 \\ \mathrm{H} & -0.800793 & 1.432741 & 2.411356 \\ \mathrm{H} & -0.407312 & 2.825007 & 3.167575 \\ \mathrm{H} & -0.190516 & 2.680799 & 1.555404 \\ \mathrm{H} & -2.792755 & 2.499357 & -3.289493 \\ \mathrm{H} & -2.763473 & 2.636606 & 2.871050 \\ \mathrm{H} & -4.882343 & -5.123745 & 0.738624 \\ \mathrm{H} & -3.943809 & -5.651445 & 1.967004 \\ \mathrm{H} & -3.297393 & -5.420432 & 0.484437 \\ \mathrm{H} & -1.177115 & -5.021426 & 1.201668 \\ \mathrm{H} & 0.389710 & -4.973187 & 0.743468 \\ \mathrm{H} & -0.788273 & -5.067708 & -0.383465 \\ \mathrm{H} & -3.839909 & 0.350122 & -3.465266 \\ \mathrm{H} & -4.455878 & 1.391886 & -4.561855 \\ \mathrm{H} & -5.296497 & 1.042864 & -3.203699 \\ \mathrm{H} & -5.516983 & 3.389617 & -2.784399 \\ \mathrm{H} & -4.656545 & 3.716600 & -4.134703 \\ \mathrm{H} & -4.213036 & 4.368889 & -2.704524 \\ \mathrm{H} & -2.051483 & 4.786384 & -2.281618 \\ \mathrm{H} & -0.720462 & 5.341717 & -1.512989 \\ \mathrm{H} & -2.142257 & 5.234141 & -0.714414 \\ \mathrm{H} & -1.592850 & 4.850513 & 1.445706 \\ \mathrm{H} & -1.846620 & 4.774827 & 3.056606 \\ \mathrm{H} & -3.116574 & 4.831582 & 2.031626\end{array}$

Table S42. Coordinates for optimized structure of 2-N,O.

$\begin{array}{cccc}\mathrm{U} & -2.744837 & -0.117787 & 0.003054 \\ \mathrm{~F} & 7.825205 & -1.073722 & -1.972833 \\ \mathrm{~F} & 2.297686 & 1.904258 & 1.937940 \\ \mathrm{~F} & 4.632650 & 2.105474 & -2.177591 \\ \mathrm{~F} & 5.097101 & 1.471566 & 2.199754 \\ \mathrm{~F} & 3.081488 & -2.534979 & 0.658074 \\ \mathrm{~F} & 6.238846 & 0.414840 & -0.404666 \\ \mathrm{~F} & 4.132867 & -3.838490 & 2.722669 \\ \mathrm{~F} & 6.744841 & -2.960064 & -3.599328 \\ \mathrm{~F} & 1.934221 & 4.553853 & 1.837932 \\ \mathrm{~F} & 6.055897 & 0.167363 & 4.286650 \\ \mathrm{~F} & 2.447839 & -1.728277 & -2.244183 \\ \mathrm{~F} & 2.905429 & 5.993265 & -0.259534 \\ \mathrm{~F} & 5.616732 & -2.504016 & 4.586755 \\ \mathrm{~F} & 4.269887 & 4.725681 & -2.243084 \\ \mathrm{~F} & 4.031635 & -3.202016 & -3.761705 \\ \mathrm{O} & -0.440105 & 0.156640 & -0.098454\end{array}$




$\begin{array}{rrrc}\mathrm{N} & 1.916845 & -0.089167 & -0.147450 \\ \mathrm{O} & -2.270768 & -1.115960 & 4.443007 \\ \mathrm{~N} & -2.699708 & -0.423801 & 2.225592 \\ \mathrm{C} & -1.895137 & -2.686440 & -0.215474 \\ \mathrm{C} & 4.787594 & 0.160111 & 2.293870 \\ \mathrm{C} & -3.248357 & -2.845393 & 0.254776 \\ \mathrm{C} & -0.773540 & -1.961838 & -2.493360 \\ \mathrm{H} & -0.006261 & -1.604045 & -1.959980 \\ \mathrm{C} & 0.775867 & 0.032596 & -0.118598 \\ \mathrm{C} & -4.213978 & 1.960111 & 0.831940 \\ \mathrm{C} & 3.949892 & 2.646214 & -1.148797 \\ \mathrm{C} & -2.462813 & 2.617363 & -0.540031 \\ \mathrm{C} & 4.268750 & -0.565141 & -1.245407 \\ \mathrm{C} & 2.596620 & 3.933201 & 0.845022 \\ \mathrm{C} & -4.584142 & 1.755039 & -0.512454 \\ \mathrm{H} & -5.414550 & 1.394067 & -0.800365 \\ \mathrm{C} & 4.004938 & -0.451025 & 1.328908 \\ \mathrm{C} & -1.945385 & -2.172897 & -1.544558 \\ \mathrm{C} & 3.471011 & 1.857470 & -0.122917 \\ \mathrm{C} & 3.086961 & 4.670117 & -0.212232 \\ \mathrm{C} & 5.096652 & -1.847070 & 3.535607 \\ \mathrm{C} & -3.616208 & -3.618055 & 1.506890 \\ \mathrm{H} & -2.797475 & -3.646875 & 2.081864 \\ \mathrm{C} & -2.485951 & -0.749607 & 3.324842 \\ \mathrm{C} & -3.304582 & -1.973503 & -1.860137 \\ \mathrm{H} & -3.628780 & -1.612613 & -2.676748 \\ \mathrm{C} & -4.115067 & -2.390113 & -0.777968 \\ \mathrm{C} & -5.092034 & 1.722555 & 2.043318 \\ \mathrm{H} & -4.504716 & 1.396579 & 2.783956 \\ \mathrm{C} & 3.775477 & -1.501733 & -2.135149 \\ \mathrm{C} & -3.537686 & 2.161241 & -1.369756 \\ \mathrm{C} & 2.802932 & 2.570933 & 0.862966 \\ \mathrm{C} & 4.342182 & -2.516616 & 2.601038 \\ \mathrm{C} & -4.749832 & -3.065458 & 2.377969 \\ \mathrm{H} & -4.619670 & -2.104959 & 2.513976 \\ \mathrm{H} & -4.745731 & -3.521774 & 3.244870 \\ \mathrm{H} & -5.607572 & -3.218532 & 1.931588 \\ \mathrm{C} & -5.622788 & -2.580100 & -0.889575 \\ \mathrm{H} & -5.996754 & -2.683340 & 0.032547 \\ \mathrm{C} & -2.880557 & 2.478241 & 0.819327 \\ \mathrm{C} & -6.339932 & -1.426558 & -1.570228 \\ \mathrm{H} & -6.210569 & -0.607218 & -1.048796 \\ \mathrm{H} & -7.296618 & -1.627673 & -1.631069 \\ \mathrm{H} & -5.975161 & -1.301475 & -2.471216 \\ \mathrm{C} & 3.773014 & 4.015137 & -1.209700 \\ \mathrm{C} & -0.228318 & -2.592627 & 1.811337\end{array}$




\begin{tabular}{|c|c|c|c|}
\hline & 264 & & \\
\hline & & & \\
\hline & 57580 & 2 & \\
\hline & & & \\
\hline & 049 & 05 & \\
\hline & & & \\
\hline & 689 & -3. & \\
\hline & -0.5 & & \\
\hline & & -2. & \\
\hline & -6.1 & 0. & \\
\hline & & & \\
\hline & -6.6 & & \\
\hline & & & \\
\hline & & -1 & \\
\hline & & & \\
\hline & & -1.2 & \\
\hline & & & \\
\hline & & & -2 \\
\hline & & & \\
\hline & & & \\
\hline & & & \\
\hline & & -3 & \\
\hline & & -3 . & \\
\hline & & -4 & \\
\hline & & -4 & \\
\hline & & & \\
\hline & & -1 & \\
\hline & -0.2 & -0. & \\
\hline & & -0 . & 166 \\
\hline & & -0.5 & \\
\hline & & -2.2 & -2 \\
\hline & & & \\
\hline & -5. & & \\
\hline & & & \\
\hline & & & \\
\hline & -1 . & & -0 \\
\hline & & & \\
\hline & -0.7 & & \\
\hline & & & \\
\hline & -0 & & \\
\hline & & & \\
\hline & & & \\
\hline & & 2.4 & -3.2 \\
\hline & & 0.2 & -0.063745 \\
\hline & -2.1 & 2.9 & 2.087895 \\
\hline & & 2.62555 & 2.85601 \\
\hline
\end{tabular}




$\begin{array}{lrrr}\mathrm{C} & -3.947609 & -5.071030 & 1.129371 \\ \mathrm{H} & -4.844314 & -5.109894 & 0.734136 \\ \mathrm{H} & -3.921342 & -5.632267 & 1.932303 \\ \mathrm{H} & -3.291527 & -5.396644 & 0.480251 \\ \mathrm{C} & -0.521207 & -4.671600 & 0.488919 \\ \mathrm{H} & -1.143756 & -4.998210 & 1.170864 \\ \mathrm{H} & 0.389167 & -4.953014 & 0.715715 \\ \mathrm{H} & -0.768979 & -5.040407 & -0.383646 \\ \mathrm{C} & -4.368582 & 1.162497 & -3.579215 \\ \mathrm{H} & -3.834297 & 0.350224 & -3.456025 \\ \mathrm{H} & -4.442817 & 1.364331 & -4.534737 \\ \mathrm{H} & -5.264257 & 1.026306 & -3.202331 \\ \mathrm{C} & -4.593952 & 3.528225 & -3.151831 \\ \mathrm{H} & -5.490056 & 3.360852 & -2.794873 \\ \mathrm{H} & -4.648666 & 3.678884 & -4.118126 \\ \mathrm{H} & -4.215094 & 4.324195 & -2.719485 \\ \mathrm{C} & -1.547430 & 4.790386 & -1.407545 \\ \mathrm{H} & -2.026063 & 4.769771 & -2.261770 \\ \mathrm{H} & -0.723180 & 5.312502 & -1.506933 \\ \mathrm{H} & -2.116770 & 5.206523 & -0.725907 \\ \mathrm{C} & -2.176874 & 4.463599 & 2.158147 \\ \mathrm{H} & -1.595822 & 4.820049 & 1.454609 \\ \mathrm{H} & -1.842391 & 4.748540 & 3.033032 \\ \mathrm{H} & -3.087717 & 4.800042 & 2.031089\end{array}$

Table S43. Coordinates for optimized structure of $\mathbf{2 - O}, \mathbf{N}$.

$\begin{array}{lrrr}C & 4.271362 & -2.350013 & 0.453743 \\ \mathrm{C} & 3.380259 & -2.147626 & 1.546915 \\ \mathrm{C} & 2.058107 & -2.476250 & 1.157111 \\ \mathrm{C} & 2.101675 & -2.807419 & -0.237695 \\ \mathrm{C} & 3.480638 & -2.746568 & -0.667003 \\ \mathrm{C} & 0.888846 & -2.624507 & 2.111330 \\ \mathrm{C} & 0.764486 & -4.087591 & 2.571404 \\ \mathrm{C} & 0.893500 & -3.340830 & -1.002838 \\ \mathrm{C} & 0.536618 & -2.651583 & -2.329746 \\ \mathrm{C} & 3.982282 & -3.281386 & -2.003166 \\ \mathrm{C} & 4.965349 & -2.404024 & -2.789468 \\ \mathrm{C} & 5.773499 & -2.512374 & 0.622199 \\ \mathrm{C} & 6.490904 & -1.340250 & 1.294292 \\ \mathrm{C} & 0.985487 & -4.862762 & -1.222184 \\ \mathrm{C} & 4.576502 & -4.693592 & -1.835043 \\ \mathrm{C} & 0.992064 & -1.708127 & 3.332904 \\ \mathrm{C} & 6.043580 & -3.800838 & 1.423165 \\ \mathrm{U} & 2.617266 & -0.117528 & -0.054524\end{array}$




\begin{tabular}{|c|c|c|c|}
\hline & & & \\
\hline & & & \\
\hline & 165 & 64 & \\
\hline & 89 & & \\
\hline & 179 & & \\
\hline & 333 & & \\
\hline & 444 & & \\
\hline & 9348 & 362 & \\
\hline & 5959 & 003 & \\
\hline & 032 & -1 . & \\
\hline & -6.2 & & \\
\hline & -5.2 & & \\
\hline & -4.4 & & \\
\hline & -5.6 & & \\
\hline & -7.2 & & \\
\hline & -7.0 & & \\
\hline & & & \\
\hline 1 & -3.5 & -2 . & \\
\hline 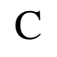 & -3.8 & & \\
\hline & -3.0 & & \\
\hline & -3.5 & & \\
\hline & -4.8 & & \\
\hline & -5.6 & & \\
\hline & -5.1 & & \\
\hline & -2.7 & & \\
\hline & & & \\
\hline & -5.9 & & \\
\hline & -6.8 & & \\
\hline & -5.2 & & \\
\hline & -3.2 & & \\
\hline & -3.3 & & \\
\hline & -3.2 & & \\
\hline & -2.9 & 58 & -2 \\
\hline & -2.8 & & \\
\hline & -3.0 & & \\
\hline & -3.5 & & \\
\hline & -3.3 & & \\
\hline & -2.81 & & \\
\hline & -2.60 & & \\
\hline & -2.87 & & -2 \\
\hline & 4.36 & & \\
\hline & 3.9 & & -0. \\
\hline & 2.63 & & -0 . \\
\hline & & & \\
\hline & 3.299322 & & 1.7 \\
\hline & 4.923293 & 2.259385 & -1.68748 \\
\hline
\end{tabular}




$\begin{array}{rrrr}\mathrm{C} & 6.060563 & 1.235515 & -1.659874 \\ \mathrm{C} & 1.917852 & 3.252243 & -1.593664 \\ \mathrm{C} & 0.564623 & 2.654796 & -2.004182 \\ \mathrm{C} & 0.925511 & 3.112838 & 1.504841 \\ \mathrm{C} & 0.100770 & 2.270151 & 2.487641 \\ \mathrm{C} & 3.487781 & 2.014814 & 3.220994 \\ \mathrm{C} & 4.073058 & 0.748128 & 3.848425 \\ \mathrm{C} & 5.505600 & 3.681067 & -1.771793 \\ \mathrm{C} & 1.205504 & 4.492814 & 2.130871 \\ \mathrm{C} & 4.388545 & 3.216802 & 3.566115 \\ \mathrm{C} & 1.778609 & 4.775196 & -1.413642 \\ \mathrm{H} & -0.038111 & -2.368448 & 1.587911 \\ \mathrm{H} & 5.355019 & 1.379087 & 1.091196 \\ \mathrm{H} & 3.098829 & -3.389051 & -2.637494 \\ \mathrm{H} & 3.691820 & -1.898188 & 2.553640 \\ \mathrm{H} & 4.351545 & 2.085967 & -2.606729 \\ \mathrm{H} & 4.568334 & -1.407624 & -2.985165 \\ \mathrm{H} & 5.166261 & -2.866379 & -3.761428 \\ \mathrm{H} & 5.930001 & -2.295499 & -2.285023 \\ \mathrm{H} & 6.215649 & -2.641655 & -0.369315 \\ \mathrm{H} & 6.427715 & -0.430195 & 0.694471 \\ \mathrm{H} & 7.552073 & -1.574599 & 1.428242 \\ \mathrm{H} & 6.076411 & -1.133470 & 2.286143 \\ \mathrm{H} & 0.414519 & -1.572005 & -2.223220 \\ \mathrm{H} & -0.410980 & -3.053962 & -2.702707 \\ \mathrm{H} & 1.285016 & -2.818803 & -3.108924 \\ \mathrm{H} & 0.610213 & -4.766894 & 1.729520 \\ \mathrm{H} & -0.086806 & -4.198855 & 3.251154 \\ \mathrm{H} & 1.668458 & -4.406445 & 3.101858 \\ \mathrm{H} & 0.037171 & -3.179724 & -0.336929 \\ \mathrm{H} & 6.739517 & 1.412199 & -0.818239 \\ \mathrm{H} & 6.653023 & 1.304591 & -2.577041 \\ \mathrm{H} & 5.690858 & 0.206515 & -1.591145 \\ \mathrm{H} & -0.092563 & 1.266217 & 2.109906 \\ \mathrm{H} & -0.866495 & 2.755600 & 2.656250 \\ \mathrm{H} & 0.579324 & 2.177851 & 3.466971 \\ \mathrm{H} & 5.692010 & -3.694720 & 2.455128 \\ \mathrm{H} & 7.117652 & -4.014035 & 1.454858 \\ \mathrm{H} & 5.535703 & -4.664996 & 0.987462 \\ \mathrm{H} & 1.857148 & -1.965387 & 3.954362 \\ \mathrm{H} & 0.099711 & -1.807008 & 3.956097 \\ \mathrm{H} & 1.070093 & -0.652175 & 3.052316 \\ \mathrm{H} & 4.720078 & 4.435410 & -1.862642 \\ \mathrm{H} & 6.161086 & 3.772833 & -2.644220 \\ \mathrm{H} & 6.093791 & 3.912462 & -0.876956 \\ \mathrm{H} & 0.283109 & 3.284359 & 0.636454\end{array}$




$\begin{array}{rrrr}\mathrm{H} & 0.635897 & 1.589751 & -2.233981 \\ \mathrm{H} & 0.212184 & 3.159170 & -2.909680 \\ \mathrm{H} & -0.199878 & 2.782502 & -1.232408 \\ \mathrm{H} & 2.512833 & 2.190190 & 3.682174 \\ \mathrm{H} & 2.578014 & 3.103344 & -2.455510 \\ \mathrm{H} & 5.549190 & -4.661999 & -1.335046 \\ \mathrm{H} & 4.730399 & -5.152696 & -2.817579 \\ \mathrm{H} & 3.921153 & -5.348202 & -1.254338 \\ \mathrm{H} & 1.713378 & -5.114739 & -1.999017 \\ \mathrm{H} & 0.014260 & -5.251754 & -1.545582 \\ \mathrm{H} & 1.273262 & -5.390784 & -0.308740 \\ \mathrm{H} & 3.401546 & -0.106198 & 3.739947 \\ \mathrm{H} & 4.240180 & 0.900727 & 4.919659 \\ \mathrm{H} & 5.039023 & 0.492274 & 3.401378 \\ \mathrm{H} & 5.406318 & 3.057079 & 3.194061 \\ \mathrm{H} & 4.445189 & 3.352097 & 4.651659 \\ \mathrm{H} & 4.016455 & 4.144907 & 3.124916 \\ \mathrm{H} & 1.730991 & 4.393954 & 3.085488 \\ \mathrm{H} & 0.261502 & 5.011623 & 2.329847 \\ \mathrm{H} & 1.810163 & 5.127232 & 1.477875 \\ \mathrm{H} & 1.003519 & 5.028579 & -0.684496 \\ \mathrm{H} & 1.493407 & 5.235867 & -2.365320 \\ \mathrm{H} & 2.714085 & 5.236055 & -1.082868\end{array}$

Table S44. Coordinates for optimized structure of $\mathbf{2}-\mathrm{N}_{3}$.

$\begin{array}{crcc}\text { U } & 5.596415 & 6.879664 & 5.554409 \\ \mathrm{~F} & 13.974437 & 9.080422 & 6.862035 \\ \mathrm{~F} & 13.644716 & 6.470350 & 8.379672 \\ \mathrm{~F} & 10.484075 & 6.206442 & 4.854097 \\ \mathrm{~F} & 9.811796 & 10.352666 & 6.799925 \\ \mathrm{~F} & 10.243008 & 8.502124 & 9.734303 \\ \mathrm{~F} & 12.946758 & 7.796986 & 4.321642 \\ \mathrm{~F} & 15.215285 & 10.488182 & 8.779203 \\ \mathrm{~F} & 13.989371 & 10.900251 & 11.189152 \\ \mathrm{~F} & 14.540587 & 4.056612 & 7.741616 \\ \mathrm{~N} & 8.816909 & 7.607189 & 6.776720 \\ \mathrm{~F} & 11.388662 & 3.759997 & 4.240201 \\ \mathrm{~N} & 9.856540 & 7.588294 & 7.311003 \\ \mathrm{~F} & 11.309890 & 11.832731 & 2.606792 \\ \mathrm{~F} & 11.493707 & 9.881716 & 11.618781 \\ \mathrm{~F} & 13.442988 & 2.657493 & 5.673598 \\ \mathrm{~N} & 6.501221 & 5.455975 & 4.170783 \\ \mathrm{~N} & 7.024065 & 4.615564 & 3.472437 \\ \mathrm{~N} & 7.732033 & 7.595538 & 6.344515\end{array}$




\begin{tabular}{|c|c|c|c|}
\hline $\mathrm{F}$ & 70510 & 12.176498 & 798 \\
\hline $\mathrm{F}$ & 12.891581 & 9.621252 & 2.391050 \\
\hline $\mathrm{C}$ & 4.969238 & 8.168088 & 3.099509 \\
\hline C & 12.030357 & 6.475977 & 6.624312 \\
\hline $\mathrm{C}$ & 11.355819 & 8.966301 & 5.651041 \\
\hline $\mathrm{C}$ & 5.613668 & 5.773235 & 8.191132 \\
\hline $\mathrm{C}$ & 6.063095 & 8.880090 & 3.717332 \\
\hline $\mathrm{C}$ & 13.060185 & 5.862228 & 7.332331 \\
\hline $\mathrm{C}$ & 3.811068 & 8.351103 & 3.915951 \\
\hline $\mathrm{C}$ & 13.322158 & 9.277018 & 8.025349 \\
\hline $\mathrm{C}$ & 5.779233 & 4.702854 & 7.237774 \\
\hline $\mathrm{C}$ & 11.471114 & 8.967002 & 9.431932 \\
\hline $\mathrm{C}$ & 4.204557 & 9.136548 & 5.036305 \\
\hline $\mathrm{H}$ & 3.529642 & 9.488803 & 5.806501 \\
\hline $\mathrm{C}$ & 12.992680 & 3.877578 & 5.973775 \\
\hline $\mathrm{C}$ & 4.301858 & 6.311985 & 8.017276 \\
\hline $\mathrm{C}$ & 6.914960 & 3.691333 & 7.125950 \\
\hline $\mathrm{H}$ & 6.664484 & 3.072407 & 6.256445 \\
\hline $\mathrm{C}$ & 6.574659 & 6.055599 & 9.340858 \\
\hline $\mathrm{H}$ & 7.518908 & 5.572343 & 9.073958 \\
\hline $\mathrm{C}$ & 12.104111 & 9.686925 & 10.442425 \\
\hline $\mathrm{C}$ & 6.912927 & 7.524953 & 9.632351 \\
\hline $\mathrm{H}$ & 7.182274 & 8.079411 & 8.732022 \\
\hline $\mathrm{H}$ & 7.766584 & 7.577382 & 10.314024 \\
\hline $\mathrm{H}$ & 6.087182 & 8.056656 & 10.114236 \\
\hline $\mathrm{C}$ & 12.047871 & 8.733523 & 8.187216 \\
\hline $\mathrm{C}$ & 4.562042 & 4.583970 & 6.489815 \\
\hline $\mathrm{C}$ & 10.548350 & 11.092922 & 4.717888 \\
\hline $\mathrm{C}$ & 5.022454 & 7.579813 & 1.694721 \\
\hline $\mathrm{H}$ & 6.082206 & 7.523217 & 1.432873 \\
\hline $\mathrm{C}$ & 10.583870 & 10.124900 & 5.713041 \\
\hline $\mathrm{C}$ & 2.362146 & 8.137108 & 3.509514 \\
\hline $\mathrm{H}$ & 2.346889 & 7.474467 & 2.639734 \\
\hline $\mathrm{C}$ & 8.320989 & 4.242435 & 6.850487 \\
\hline $\mathrm{H}$ & 8.341584 & 4.874340 & 5.959913 \\
\hline $\mathrm{H}$ & 9.009020 & 3.407930 & 6.677674 \\
\hline $\mathrm{H}$ & 8.723334 & 4.817333 & 7.690196 \\
\hline B & 11.380742 & 7.942604 & 6.929822 \\
\hline $\mathrm{C}$ & 11.502406 & 5.705027 & 5.590415 \\
\hline $\mathrm{C}$ & 5.567324 & 9.509508 & 4.904944 \\
\hline $\mathrm{C}$ & 12.131679 & 8.847949 & 4.498806 \\
\hline $\mathrm{C}$ & 4.183505 & 3.475975 & 5.526021 \\
\hline $\mathrm{H}$ & 5.084119 & 3.157970 & 4.987482 \\
\hline C & 13.990786 & 9.998938 & 9.002520 \\
\hline $\mathrm{C}$ & 3.686211 & 5.603300 & 6.946931 \\
\hline I & 2.657938 & 5.735803 & 6.634742 \\
\hline
\end{tabular}




$\begin{array}{cccc}\mathrm{C} & 11.330528 & 10.922466 & 3.583263 \\ \mathrm{C} & 13.371834 & 10.207149 & 10.229230 \\ \mathrm{C} & 11.949556 & 4.439372 & 5.246979 \\ \mathrm{C} & 3.528011 & 7.174066 & 9.001410 \\ \mathrm{H} & 4.240059 & 7.596359 & 9.714794 \\ \mathrm{C} & 4.463112 & 6.163502 & 1.508148 \\ \mathrm{H} & 4.928184 & 5.450284 & 2.189902 \\ \mathrm{H} & 4.658955 & 5.824050 & 0.485498 \\ \mathrm{H} & 3.380243 & 6.116308 & 1.656629 \\ \mathrm{C} & 12.130451 & 9.792595 & 3.476342 \\ \mathrm{C} & 13.548525 & 4.594113 & 7.023520 \\ \mathrm{C} & 3.157180 & 3.913768 & 4.479001 \\ \mathrm{H} & 2.193629 & 4.157833 & 4.939661 \\ \mathrm{H} & 2.977229 & 3.106819 & 3.761726 \\ \mathrm{H} & 3.496368 & 4.786014 & 3.909967 \\ \mathrm{~N} & 7.514843 & 3.826357 & 2.805605 \\ \mathrm{C} & 4.367234 & 8.528488 & 0.672150 \\ \mathrm{H} & 3.276957 & 8.525462 & 0.763289 \\ \mathrm{H} & 4.609520 & 8.205819 & -0.346307 \\ \mathrm{H} & 4.711272 & 9.559270 & 0.790699 \\ \mathrm{C} & 1.765057 & 9.489422 & 3.073989 \\ \mathrm{H} & 1.682530 & 10.167939 & 3.929961 \\ \mathrm{H} & 0.760972 & 9.349379 & 2.658643 \\ \mathrm{H} & 2.382348 & 9.982670 & 2.318992 \\ \mathrm{C} & 6.958248 & 2.740031 & 8.335542 \\ \mathrm{H} & 7.357472 & 3.238444 & 9.223231 \\ \mathrm{H} & 7.611052 & 1.887968 & 8.117990 \\ \mathrm{H} & 5.966970 & 2.351435 & 8.586426 \\ \mathrm{C} & 7.451979 & 9.157728 & 3.152765 \\ \mathrm{H} & 7.975858 & 9.708756 & 3.942701 \\ \mathrm{C} & 6.171429 & 11.929610 & 5.137022 \\ \mathrm{H} & 6.593027 & 11.955765 & 4.129544 \\ \mathrm{H} & 6.711277 & 12.664799 & 5.742592 \\ \mathrm{H} & 5.123057 & 12.241713 & 5.072944 \\ \mathrm{C} & 3.651126 & 2.256708 & 6.298162 \\ \mathrm{H} & 4.391663 & 1.867354 & 7.001346 \\ \mathrm{H} & 3.387755 & 1.449567 & 5.605960 \\ \mathrm{H} & 2.753709 & 2.522143 & 6.867480 \\ \mathrm{C} & 8.340312 & 7.946677 & 2.832261 \\ \mathrm{H} & 8.467759 & 7.287174 & 3.691932 \\ \mathrm{H} & 9.334831 & 8.296291 & 2.535820 \\ \mathrm{H} & 7.955353 & 7.345038 & 2.003838 \\ \mathrm{C} & 5.773956 & 10.575960 & 7.209458 \\ \mathrm{H} & 4.737138 & 10.927602 & 7.258130 \\ \mathrm{H} & 6.379899 & 11.268770 & 7.801193 \\ \mathrm{H} & 5.827673 & 9.599004 & 7.701951\end{array}$




$\begin{array}{cccc}\mathrm{C} & 6.087539 & 5.386263 & 10.640979 \\ \mathrm{H} & 5.208953 & 5.899261 & 11.043604 \\ \mathrm{H} & 6.872042 & 5.434570 & 11.403910 \\ \mathrm{H} & 5.823119 & 4.336465 & 10.490833 \\ \mathrm{C} & 6.283966 & 10.530860 & 5.767261 \\ \mathrm{H} & 7.346958 & 10.270364 & 5.809885 \\ \mathrm{C} & 7.393827 & 10.099038 & 1.935064 \\ \mathrm{H} & 7.027336 & 9.582695 & 1.043026 \\ \mathrm{H} & 8.396405 & 10.474110 & 1.703297 \\ \mathrm{H} & 6.745220 & 10.961204 & 2.114372 \\ \mathrm{C} & 1.464137 & 7.510701 & 4.577414 \\ \mathrm{H} & 1.790515 & 6.502070 & 4.838663 \\ \mathrm{H} & 0.434401 & 7.441637 & 4.211291 \\ \mathrm{H} & 1.444476 & 8.117528 & 5.488271 \\ \mathrm{C} & 2.752273 & 8.340456 & 8.386622 \\ \mathrm{H} & 3.420276 & 9.070946 & 7.925277 \\ \mathrm{H} & 2.178898 & 8.861364 & 9.160569 \\ \mathrm{H} & 2.039662 & 7.994071 & 7.631168 \\ \mathrm{C} & 2.558637 & 6.274413 & 9.792039 \\ \mathrm{H} & 2.074213 & 6.844386 & 10.592450 \\ \mathrm{H} & 1.773266 & 5.881030 & 9.137470 \\ \mathrm{H} & 3.072660 & 5.421228 & 10.242476\end{array}$

Table S45. Coordinates for optimized structure of free $\mathrm{N}_{3}{ }^{-}$.

$\begin{array}{llll}\mathrm{N} & 7.267633 & 6.233512 & 4.357063 \\ \mathrm{~N} & 8.098473 & 6.906759 & 3.878872 \\ \mathrm{~N} & 8.929064 & 7.580029 & 3.400345\end{array}$

Table S46. Coordinates for optimized structure of free $\mathrm{NCO}^{-}$.
C $\quad 5.893903$
4.167788
0.113013
O 7.105209
4.395008
0.117795
N $\quad 4.717898 \quad 3.947566 \quad 0.107762$ 


\section{References}

(1) Boreen, M. A.; Lussier, D. J.; Skeel, B. A.; Lohrey, T. D.; Watt, F. A.; Shuh, D. K.; Long, J. R.; Hohloch, S.; Arnold, J. Structural, Electrochemical, and Magnetic Studies of Bulky Uranium(III) and Uranium(IV) Metallocenes. Inorg. Chem. 2019, 58, 16629.

(2) Gazis, T. A.; Dasgupta, A.; Hill, M. S.; Rawson, J. M.; Wirth, T.; Melen, R. L. Reactions of hydrazones and hydrazides with Lewis acidic boranes. Dalton Trans. 2019, 48, 12391.

(3) Lawrence, E. J.; Oganesyan, V. S.; Wildgoose, G. G.; Ashley, A. E. Exploring the fate of the tris(pentafluorophenyl)borane radical anion in weakly coordinating solvents. Dalton Trans. 2013, $42,782$.

(4) LeSuer, R. J.; Buttolph, C.; Geiger, W. E. Comparison of the Conductivity Properties of the Tetrabutylammonium Salt of Tetrakis(pentafluorophenyl)borate Anion with Those of Traditional Supporting Electrolyte Anions in Nonaqueous Solvents. Anal. Chem. 2004, 76, 6395.

(5) Boreen, M. A.; Rao, G.; Villarreal, D. G.; Watt, F. A.; Britt, R. D.; Hohloch, S.; Arnold, J. Lewis acid capping of a uranium(V) nitride via a uranium(III) azide molecular square. Chem. Commun. 2020, 56, 4535.

(6) Bruker. APEX2, APEX3, and SAINT. Bruker AXS Inc., Madison, Wisconsin, USA.

(7) Bruker. SADABS. Bruker AXS Inc., Madison, Wisconsin, USA.

(8) Rigaku Oxford Diffraction, (2015), CrysAlisPro Software system, version 1.171.39.7a, Rigaku Corporation, Oxford, UK.

(9) Sheldrick, G. M. SHELXT - Integrated space-group and crystal-structure determination. Acta Cryst. 2015, A71, 3.

(10) Sheldrick, G. M. A short history of SHELX. Acta Cryst. 2008, A64, 112.

(11) Dolomanov, O. V.; Bourhis, L. J.; Gildea, R. J.; Howard, J. A. K.; Puschmann, H. OLEX2: a complete structure solution, refinement and analysis program. J. Appl. Cryst. 2009, 42, 339.

(12) Farrugia, L. J. WinGX and ORTEP for Windows: an update. J. Appl. Cryst. 2012, 45, 849.

(13) Macrae, C. F.; Bruno, I. J.; Chisholm, J. A.; Edgington, P. R.; McCabe, P.; Pidcock, E.; Rodriguez-Monge, L.; Taylor, R.; van de Streek, J.; Wood, P. A. Mercury CSD 2.0 - new features 
for the visualization and investigation of crystal structures. J. Appl. Cryst. 2008, 41, 466.

(14) Spek, A. L. PLATON SQUEEZE: a tool for the calculation of the disordered solvent contribution to the calculated structure factors. Acta Cryst. 2015, C71, 9.

(15) van der Sluis, P.; Spek, A. L. BYPASS: an effective method for the refinement of crystal structures containing disordered solvent regions. Acta Cryst. 1990, A46, 194.

(16) Frey, A. S. P.; Cloke, F. G. N.; Coles, M. P.; Hitchcock, P. B. UIII-Induced Reductive CoCoupling of $\mathrm{NO}$ and $\mathrm{CO}$ to Form $\mathrm{U}^{\mathrm{IV}}$ Cyanate and Oxo Derivates. Chem. - Eur. J. 2010, 16, 9446.

(17) Becke, A. D. Density-functional thermochemistry. III. The role of exact exchange. J. Chem. Phys. 1993, 98, 5648.

(18) Perdew, J. P.; Wang, Y. Accurate and simple analytic representation of the electron-gas correlation energy. Phys. Rev. B 1992, 45, 13244.

(19) Küchle, W.; Dolg, M.; Stoll, H.; Preuss, H. Energy-adjusted pseudopotentials for the actinides. Parameter sets and test calculations for thorium and thorium monoxide. J. Chem. Phys. 1994, 100, 7535.

(20) Cao, X.; Dolg, M.; Stoll, H. Valence basis sets for relativistic energy-consistent small-core actinide pseudopotentials. J. Chem. Phys. 2003, 118, 487.

(21) Ditchfield, R.; Hehre, W. J.; Pople, J. A. Self-Consistent Molecular-Orbital Methods. IX. An Extended Gaussian-Type Basis for Molecular-Orbital Studies of Organic Molecules. J. Chem. Phys. 1971, 54, 724.

(22) Hehre, W. J.; Ditchfield, R.; Pople, J. A. Self-Consistent Molecular Orbital Methods. XII. Further Extensions of Gaussian-Type Basis Sets for Use in Molecular Orbital Studies of Organic Molecules. J. Chem. Phys. 1972, 56, 2257.

(23) Hariharan, P. C.; Pople, J. A. The Influence of Polarization Functions on Molecular Orbital Hydrogenation Energies. Theor. Chim. Acta 1973, 28, 213.

(24) Gaussian 09, Revision D.01. Frisch, M. J.; Trucks, G. W.; Schlegel, H. B.; Scuseria, G. E.; Robb, M. A.; Cheeseman, J. R.; Scalmani, G.; Barone, V.; Mennucci, B.; Petersson, G. A.; Nakatsuji, H.; Caricato, M.; Li, X.; Hratchian, H. P.; Izmaylov, A. F.; Bloino, J.; Zheng, G.; Sonnenberg, J. L.; Hada, M.; Ehara, M.; Toyota, K.; Fukuda, R.; Hasegawa, J.; Ishida, M.; Nakajima, T.; Honda, Y.; Kitao, O.; Nakai, H.; Vreven, T.; Montgomery, J. A., Jr.; Peralta, J. E.; 
Ogliaro, F.; Bearpark, M.; Heyd, J. J.; Brothers, E.; Kudin, K. N.; Staroverov, V. N.; Kobayashi, R.; Normand, J.; Raghavachari, K.; Rendell, A.; Burant, J. C.; Iyengar, S. S.; Tomasi, J.; Cossi, M.; Rega, N.; Millam, J. M.; Klene, M.; Knox, J. E.; Cross, J. B.; Bakken, V.; Adamo, C.; Jaramillo, J.; Gomperts, R.; Stratmann, R. E.; Yazyev, O.; Austin, A. J.; Cammi, R.; Pomelli, C.; Ochterski, J. W.; Martin, R. L.; Morokuma, K.; Zakrzewski, V. G.; Voth, G. A.; Salvador, P.; Dannenberg, J. J.; Dapprich, S.; Daniels, A. D.; Farkas, Ö.; Foresman, J. B.; Ortiz, J. V.; Cioslowski, J.; Fox, D. J. Gaussian, Inc., Wallingford CT, 2009.

(25) Reed, A. E.; Curtiss, L. A.; Weinhold, F. Intermolecular Interactions from a Natural Bond Orbital, Donor-Acceptor Viewpoint. Chem. Rev. 1988, 88, 899. 